\title{
General dynamical sharp-interface conditions for phase transformations in viscous heat-conducting fluids
}

\author{
DANIEL M. ANDERSON ${ }^{1}$, PAOLO CERMELLI I \\ ELIOT FRIED ${ }^{3}$, MORTON E. GURTIN ${ }^{4}$ \\ AND GEOFFREY B. MCFADDEN ${ }^{5}$ \\ ${ }^{1}$ Department of Mathematical Sciences, George Mason University, \\ Fairfax, VA 22030-4422, USA \\ ${ }^{2}$ Dipartimento di Matematica, Università di Torino, \\ Via Carlo Alberto 10, 10123 Torino, Italy \\ ${ }^{3}$ Department of Mechanical and Aerospace Engineering, Washington University in St Louis, \\ St Louis, MO 63130-4899, USA \\ ${ }^{4}$ Department of Mathematical Sciences, Carnegie Mellon University, \\ Pittsburgh, PA 15213-3890, USA \\ ${ }^{5}$ Mathematical and Computational Sciences Division, National Institute of Standards \\ and Technology, Gaithersburg, MD 20899-8910, USA
}

(Received 26 April 2005 and in revised form 28 December 2006)

We develop a complete set of equations governing the evolution of a sharp interface separating two fluid phases undergoing transformation. In addition to the conventional balances for mass, linear momentum and energy these equations include also a counterpart of the Gibbs-Thomson equation familiar from theories for crystal growth. This additional equation arises from a consideration of configurational forces within a thermodynamical framework. Although the notion of configurational forces is well-developed and understood for the description of materials, such as crystalline solids, that possess natural reference configurations, little has been done regarding their role in materials, such as viscous fluids, that do not possess preferred reference states. We therefore provide a comprehensive discussion of configurational forces, the balance of configurational momentum, and configurational thermodynamics that does not require a choice of reference configuration. The general evolution equations arising from our theory account for the thermodynamic structure of the bulk phases and the interface and for viscous and thermal dissipation in the bulk phases and for viscous dissipation on the interface. Because of the complexity of these equations, we provide a reduced system of equations based on simplified constitutive assumptions and approximations common in the literature on phase transformations. Using these reduced equations, we apply the theory to the radially symmetric problem for the condensation of a liquid drop into the vapour phase.

\section{Introduction}

The purpose of this paper is to develop, from basic considerations, a complete set of equations governing the evolution of a sharp interface separating two fluid phases undergoing transformation. The dynamics of two fluid phases and the evolution of the interface separating them has been a problem of scientific and industrial interest for centuries. Such problems range from those of geophysics involving large length 
scales, where inertial and gravitational effects prevail, to those involving small length scales, where surface tension and other interfacial phenomena are important. For situations in which a phase transformation does not occur, so that the interface is a material surface, the governing bulk and interfacial equations are well-developed and agreed upon (Slattery 1990; Edwards, Brenner \& Wassan 1991; Joseph \& Renardy 1993). It is important to emphasize the distinction between non-material and material interfaces: whereas non-material interfaces allow for exchanges of mass between adjacent phases, fluid particles cannot cross a material interface. Focusing on the interface, apart from appropriate kinematical conditions, the relevant equations are the conventional balances for mass, linear momentum, and energy, augmented by suitable constitutive equations. However, and what is most relevant to our discussion, when a phase transformation does occur there is an additional kinematical degree of freedom represented by the motion of the interface relative to the material; because of this, the interfacial expressions for balance of mass, momentum and energy fail to provide a closed description: an additional interface condition is needed to account for the microphysics associated with the exchange of material between phases.

The need for a supplemental equation at a sharp phase-interface was recognized early on by Lamé \& Clapeyron (1831) and, somewhat later, by Stefan (1889) who, in their works on the solidification of a pure substance, not only balanced energy in the bulk phases and on the interface, but also required that, on the interface, the temperature $\vartheta$ be equal to the melting temperature $\vartheta_{0}$ :

$$
\vartheta=\vartheta_{0} .
$$

Despite its intuitive appeal, the physical basis of (1.1) is ambiguous: is it a balance, a constitutive equation, or neither?

To address this question, we consider not (1.1) but a familiar generalization, the Gibbs-Thomson equation, in which the interfacial temperature is allowed to depend also on the curvature of the interface. Using $\mathscr{S}$ for the interface, $\boldsymbol{n}$ for its unit normal, $K=-\operatorname{div}_{g} \boldsymbol{n}$ for its total (twice the mean) curvature, $\psi^{x}=\hat{\psi}^{x}(\vartheta)$ for the interfacial free-energy (density), and $\ell$ for the latent heat, and introducing a dimensionless temperature

$$
\theta=\frac{\vartheta-\vartheta_{0}}{\vartheta_{0}},
$$

the Gibbs-Thomson equation (when the phases are of equal density $\varrho$ and flow is neglected) reads

$$
\varrho \ell \theta=\psi^{x} K .
$$

The Gibbs-Thomson equation is typically derived using a variational argument. Specifically, suppose that the medium occupies a fixed region $\mathscr{R}$ and that the interface $\mathscr{S}$ remains disjoint from the boundary $\partial \mathscr{R}$. Let $\psi=\psi^{ \pm}(\vartheta)$ denote the specific free energy of phase \pm . Since the interface is not material, its configuration can be varied independently of the temperature field. Requiring that the variation of the net free energy

$$
\int_{\mathscr{R}} \varrho \psi \mathrm{d} v+\int_{\mathscr{S}} \psi^{x} \mathrm{~d} a
$$

with respect to changes of the configuration of $\mathscr{S}$ be stationary then yields the requirement

$$
\psi^{x} K+\varrho \llbracket \psi \rrbracket=0,
$$

where $\llbracket \Phi \rrbracket=\Phi^{+}-\Phi^{-}$denotes the jump of a bulk quantity $\Phi$ across the interface. Let $\eta=\eta^{ \pm}$denote the specific entropy of phase \pm and assume (as is classical) that 
$\eta^{ \pm}=-\mathrm{d} \psi^{ \pm}(\vartheta) / \mathrm{d} \vartheta$. Assume that $\psi^{+}(\vartheta)$ and $\psi^{-}(\vartheta)$ are defined so that

$$
\psi^{+}\left(\vartheta_{0}\right)=\psi^{-}\left(\vartheta_{0}\right), \quad \eta^{+}\left(\vartheta_{0}\right) \neq \eta^{-}\left(\vartheta_{0}\right) .
$$

The Gibbs-Thomson equation (1.3) then follows from (1.5) on stipulating that the temperature difference $\vartheta-\vartheta_{0}$ deviates only infinitesimally from the melting temperature $\vartheta_{0}$ and making the identification $\ell=\vartheta_{0}\left(\eta^{+}\left(\vartheta_{0}\right)-\eta^{-}\left(\vartheta_{0}\right)\right)$.

Using the terminology of Nabarro (1985), the foregoing argument, involving as it does a variation of the configuration of the interface, distinguishes the quantity $\psi^{x} K+\varrho \llbracket \psi \rrbracket$ as the configurational force acting on $\mathscr{S}$. This answers the question posed after (1.1).

We use the adjective configurational to differentiate these forces from classical Newtonian forces, which we refer to as standard.) In equilibrium, this force vanishes. Non-trivial values of this force thus signal that the configuration of $\mathscr{S}$ is energetically suboptimal. In this vein, (1.5) and its specialization the Gibbs-Thomson equation (1.3) can be interpreted as constitutively augmented statements of configurational force balance. (Statements such as (1.5) actually represent the normal component of a vectorial configurational force balance (Gurtin 2000, p. 6). Indeed, on defining $\mathbf{C}=\varrho \psi \mathbf{1}$ and $\mathbb{C}=\psi^{x} \mathbb{P}$, with $\mathbb{P}=\mathbf{1}-\boldsymbol{n} \otimes \boldsymbol{n}$ the projection onto $\mathscr{S}$ (cf. (4.2)), a straightforward calculation relying on the identity $\operatorname{div}_{\mathscr{S}} \mathbb{P}=K \boldsymbol{n}$ (cf. (4.7)) shows that (1.5) is the normal component of the vectorial equation $\operatorname{div}_{\mathscr{S}} \mathbb{C}+\llbracket \mathbf{C} \rrbracket \boldsymbol{n}=\mathbf{0}$. In this context, $\boldsymbol{C}$ and $\mathbb{C}$ are bulk and interfacial configurational stress tensors.

That additional configurational forces may be required to describe solid-state phenomena involving evolving defect structures such as phase interfaces and grain boundaries that migrate relative to the material is clear from the groundbreaking works of Eshelby (1951, 1956, 1970, 1975), Peach \& Koehler (1950) and Herring (1951). However, as with our derivation of (1.3), these studies are based on variational arguments and by their very nature, variational arguments cannot characterize dissipation.

This drawback is particularly limiting when dealing with fluids, because of the prominent role played by viscous stresses. Finally, we note that any variationallybased introduction of configurational forces must necessarily be predicated on an underlying constitutive framework and, therefore, restricted to a particular class of materials.

That configurational forces are distinct from the standard (Newtonian) forces associated with the motion of material particles should be clear from the derivation of (1.5), which involves a variation that does not allow the fluid to move. On the other hand, variations of positions of the fluid particles - holding the interface fixed yield the conventional balance laws for standard forces.

In the dynamics of defect structures with general forms of dissipation there is no encompassing variational principle. Nevertheless, experience demonstrates the need for an additional balance associated with the kinematics of the defect. An additional balance of this sort is the relation that ensues when we formally set the variationally derived expression for the configurational force on a defect equal to a linear function of the velocity of that defect. In particular, for the solidification of a pure substance, this procedure leads to a kinetic Gibbs-Thomson equation (Voronkov 1964; see also Gurtin 1988, who uses configurational forces to derive (1.7) and its anisotropic generalization)

$$
\varrho \ell \theta=\hat{\psi}^{x}(\vartheta) K-\kappa(\vartheta) V,
$$

where $V$ denotes the scalar normal velocity of $\mathscr{S}$ and $\kappa(\vartheta) \geqslant 0$ is the reciprocal mobility of the interface. 
A completely different point of view is taken by Gurtin \& Struthers (1990, this work is somewhat obscure; better references for the key ideas are Gurtin 1995, 2000), who use an argument based on invariance under observer changes to conclude that a configurational force balance should join the standard (Newtonian) force balance as a basic law of continuum physics. Here the operative word is 'basic'. Basic laws are by their very nature independent of constitutive assumptions; when placed within a thermodynamic framework, such laws allow us to use the now standard procedures of continuum thermodynamics to develop suitable constitutive theories.

We begin our study by revisiting a familiar topic: the bulk material away from the interface. This setting allows for a discussion of the first two laws in a form that accounts explicitly for power expended by configurational forces. In this setting, the configurational and standard forms of the first two laws are equivalent; even so, this setting is useful as it provides a vehicle for discussing the basic structure of these laws, a structure not at all transparent when discussing phase interfaces. Once this basic framework is established, we turn to the goal stated at the outset of the paper: to develop, from basic considerations, a complete set of equations governing the evolution of a sharp interface separating two fluid phases undergoing transformation.

Our treatment has the following limitations.

(i) We neglect the mass of the interface.

(ii) We do not allow for heat conduction within the interface.

(iii) We restrict attention to situations in which the absolute temperature $\vartheta$ is continuous across the interface.

(iv) We require that the tangential component $\boldsymbol{u}_{\text {tan }}$ of the fluid velocity $\boldsymbol{u}$ be continuous across the interface.

Aside from the classical bulk balances for standard forces and energy, the general governing equations consist of interface conditions expressing balance of energy, balance of standard momentum, and balance of configurational momentum; a general system of local interface conditions equivalent to these basic balances is given in $\S 10$.

The general equations are complicated and, for that reason, in $\$ 12$ we consider the equations under simplifed constitutive equations and approximate assumptions (common in the literature). In particular, letting $\hat{\psi}(\vartheta)$ denote the specific free energy in bulk, if we assume, among other hypotheses, that the interfacial free energy $\psi^{x}$ is constant and the temperature $\vartheta$ is close to its equilibrium value $\vartheta_{0}(\mathrm{cf} .(1.6 a))$, then the resulting interface conditions consist of:

(i) the energy balance

$$
\llbracket q \rrbracket \cdot n=\ell J,
$$

in which $\boldsymbol{q}$ is the bulk heat-flux vector, $\ell$ is the latent heat, and $J$ is the mass flow across $\mathscr{S}$;

(ii) the standard momentum balance

$$
\llbracket \boldsymbol{T} \rrbracket \boldsymbol{n}-J^{2} \llbracket v \rrbracket \boldsymbol{n}=-\psi^{x} K \boldsymbol{n}-\operatorname{div}_{\mathscr{S}}\{2 \alpha \mathbb{D}+\lambda(\operatorname{tr} \mathbb{D}) \mathbb{P}\},
$$

with $\boldsymbol{T}$ the stress tensor, $v$ the specific volume, $\mathbb{D}$ the superficial rate of stretch (cf. (4.17)), $\alpha$ and $\lambda$ interfacial fluid viscosities, assumed constant, and $\mathbb{P}$ the projection onto $\mathscr{S}$ (cf. (4.2));

(iii) the normal configurational momentum balance, which may be expressed in the following essentially equivalent forms:

$$
\begin{aligned}
\ell \theta= & -\boldsymbol{n} \cdot \llbracket v \boldsymbol{T} \rrbracket \boldsymbol{n}+\frac{1}{2} J^{2} \llbracket v^{2} \rrbracket-\left\langle\langle v \rangle \left\{\left\{ V^{m i g}-\beta \Delta_{g} V^{m i g}\right.\right.\right. \\
& +2 \alpha \mathbb{K}: \mathbb{D}+\lambda(\operatorname{tr} \mathbb{D}) K\},
\end{aligned}
$$




$$
\ell \theta=-\llbracket v \rrbracket \boldsymbol{n} \cdot\langle\boldsymbol{T}\rangle\rangle \boldsymbol{n}+\left\langle\langle v\rangle \hat{\psi}^{x} K-\left\langle\langle v\rangle\left\{\kappa V^{m i g}-\beta \Delta_{\mathscr{\varphi}} V^{m i g}\right\} .\right.\right.
$$

(More precisely, $(1.10 a)$ represents the normal configurational momentum balance, while $(1.10 b)$ represents a combination of the normal configurational and standard momentum balances.) Here, $\theta$ is the dimensionless temperature (1.2), $\langle v\rangle=\left(v^{+}+\right.$ $\left.v^{-}\right) / 2$ is the average specific volume at the interface,

$$
V^{m i g}=V-\langle\langle\boldsymbol{u}\rangle \cdot \boldsymbol{n}
$$

represents the average migrational velocity of the interface relative to the bulk material, $\kappa$ and $\beta$ are constant viscosities associated with the migration of the interface, $\mathbb{K}=-\operatorname{grad}_{\mathscr{T}} \boldsymbol{n}$, and $\Delta_{\mathscr{S}}$ the Laplace-Beltrami operator (surface Laplacian) (12.5). The term $\beta \Delta_{\mathscr{S}} V^{m i g}$ would seem relevant to applications involving small length scales, applications whose importance lies in the development of micro- and nanoscale fluid devices (e.g. Stone, Stroock \& Adjari 2004.)

The basic interface equations (1.8)-(1.10) demonstrate the power of working within a general framework: these balances exhibit the physical nature of - and the interaction between - different physical effects and, in addition, provide a framework within which different physical assumptions may be discussed. For example, if we neglect interfacial and migrational viscosities, the standard momentum balance becomes

$$
\llbracket \boldsymbol{T} \rrbracket \boldsymbol{n}-J^{2} \llbracket v \rrbracket \boldsymbol{n}=-\psi^{x} K \boldsymbol{n},
$$

and the equivalent statements of the configurational momentum balance have the forms

$$
\ell \theta=-\llbracket v \boldsymbol{n} \cdot \boldsymbol{T n} \rrbracket+\frac{1}{2} J^{2} \llbracket v^{2} \rrbracket, \quad \ell \theta=\left\langle\langle v\rangle \hat{\psi}^{x} K-\llbracket v \rrbracket \boldsymbol{n} \cdot\langle\langle\boldsymbol{T}\rangle \boldsymbol{n} .\right.
$$

Note that $(1.12 b)$ generalizes the Gibbs-Thomson equation, (1.3), to account for density differences between phases and for the influence of flow. Note also that, because it determines the interfacial temperature solely in terms of interfacial limits of bulk quantities, $(1.12 a)$ is the simpler of the two equations. In addition, $(1.12 a)$ shows that the classical vapour recoil effect (Hickman 1952, 1972; Palmer 1976; Burelbach, Bankoff \& Davis 1988) - which is embodied by the inertial contribution $-J^{2} \llbracket v \rrbracket n$ in the standard momentum balance (1.11) - may also influence the interfacial temperature through the term $J^{2} \llbracket v^{2} \rrbracket / 2$ in $(1.12 a)$. Whereas the vapour-recoil effect is present in the interfacial momentum balance used in fluid mechanical theories for evaporation-condensation, it does not enter the classical Hertz-Knudsen-Langmuir equation (Schrage 1953) that is typically used (in lieu of the Gibbs-Thomson equation) as an additional interface equation (Burelbach et al. 1988; Danov et al. 1998).

The challenge of developing accurate and efficient numerical methods for the solution of initial-boundary-value problems arising from sharp-interface theories has spurred on the development of regularized diffuse-interface, or phase-field, theories (e.g. J. S. Langer 1978, unpublished work; Collins \& Levine 1985; Caginalp 1986, 1989; Penrose \& Fife 1990; Fried \& Gurtin 1993, 1999; Wang et al. 1993.) This approach has recently become popular for the modelling of fluid-fluid systems (Anderson, McFadden \& Wheeler 1998, 2000). Anderson, McFadden \& Wheeler (2001) analyse a two-fluid diffuse-interface model in a sharp-interface limit and derive non-equilibrium interfacial conditions for a number of limiting cases. In addition to interfacial statements of mass, momentum and energy balance, these conditions include a non-equilibrium generalization of the Gibbs-Thomson equation. In our notation, that equation reads

$$
\left.\ell \theta=p^{-} \llbracket v \rrbracket-\frac{1}{2} J^{2} \llbracket v \rrbracket^{2}+v^{+} \psi^{x} K-\kappa\langle u\rangle\right\rangle V^{m i g}
$$


and can be derived as a specialization of our equations (1.9) and (1.10b) by setting the interfacial fluid viscosities $\alpha$ and $\lambda$ and the migrational viscosity $\beta$ to zero, using the identity $\langle\langle\boldsymbol{T}\rangle\rangle=\boldsymbol{T}^{-}+\llbracket \boldsymbol{T} \rrbracket / 2$, and neglecting viscous contributions to the stresses in the bulk phases. (Compare (1.13) with (102) of Anderson et al. (2001), whose definition of the mass flow $J$ differs from ours by a sign. In writing (1.13), we set to zero the reference pressure of Anderson et al. (2001). Their analysis provides an expression for $\kappa$ in terms of the densities and shear viscosities of the phases.) Hence, (1.13) arises in our theory on neglecting all forms of bulk and interfacial dissipation other than that associated with attachment kinetics (as embodied by the migrational viscosity $\kappa$ ). Anderson et al. (2001) refer to (1.13) as a modified Clausius-Clapeyron equation. We see here that (1.13) can be viewed alternatively as a combined expression of the balances for standard and configurational momenta. (Importantly, as in the expressions $(1.10 a)$ and $(1.10 b)$ of normal configurational momentum balance, equation (102) of Anderson et al. (2001) exhibits the influence of the recoil effect discussed in the paragraph containing (1.11) and (1.12) through the term $J^{2} \llbracket v \rrbracket^{2} / 2$.) The particular sharp-interface limit obtained by an analysis of the equations arising in a diffuse-interface theory is sensitive both to constitutive and scaling assumptions. As such, a diffuse-interface theory may generally possess a variety of sharp-interface limits. In the absence of a sound sharp-interface theory to serve as a target, the problem of developing a physically meaningful diffuse-interface theory is ill-posed. Here, we provide a sound target theory upon which to build phase-field-based regularizations.

\section{Theory for the bulk phases}

\subsection{Kinematics}

We write $\boldsymbol{u}(\boldsymbol{x}, t)$ for the velocity and

$$
\boldsymbol{L}=\operatorname{grad} \boldsymbol{u}, \quad \boldsymbol{D}=\frac{1}{2}\left(\boldsymbol{L}+\boldsymbol{L}^{\top}\right), \quad \boldsymbol{W}=\frac{1}{2}\left(\boldsymbol{L}-\boldsymbol{L}^{\top}\right)
$$

for the velocity gradient, rate of stretch, and spin. We assume that the fluid is incompressible, so that

and denote by

$$
\operatorname{div} \boldsymbol{u}=\operatorname{tr} \boldsymbol{D}=0
$$

$$
\varrho(=\text { constant in each phase }), \quad v=\frac{1}{\varrho}
$$

the mass density and specific volume.

We use a superposed dot to denote the material time-derivative; e.g. for a scalar field $\Phi(\boldsymbol{x}, t)$,

$$
\dot{\Phi}=\frac{\partial \Phi}{\partial t}+(\operatorname{grad} \Phi) \cdot \boldsymbol{u} .
$$

We consistently write $\mathscr{P}(t)$ for an arbitrarily chosen region that convects with the fluid; a consequence of balance of mass is then that, for such a region and any field $\Phi$

$$
\frac{\mathrm{d}}{\mathrm{d} t} \int_{\mathscr{P}(t)} \Phi \varrho \mathrm{d} v=\int_{\mathscr{P}(t)} \dot{\Phi} \varrho \mathrm{d} v
$$

\subsection{Standard momentum and moment of momentum balances}

We neglect external body forces. Writing $\boldsymbol{T}$ for the Cauchy stress, the standard momentum and moment of momentum balances then require that, for any bulk 
region $\mathscr{P}$, with $\boldsymbol{m}$ the outward unit normal to $\partial \mathscr{P}$,

$$
\begin{aligned}
\frac{\mathrm{d}}{\mathrm{d} t} \int_{\mathscr{P}} \varrho \boldsymbol{u} \mathrm{d} v & =\int_{\partial \mathscr{P}} \boldsymbol{T} \boldsymbol{m} \mathrm{d} a, \\
\frac{\mathrm{d}}{\mathrm{d} t} \int_{\mathscr{P}}(\boldsymbol{x}-\mathbf{0}) \times \varrho \boldsymbol{u} \mathrm{d} v & =\int_{\partial \mathscr{P}}(\boldsymbol{x}-\mathbf{0}) \times \boldsymbol{T} \boldsymbol{m} \mathrm{d} a=\mathbf{0} .
\end{aligned}
$$

These imply the local balance

$$
\varrho \dot{\boldsymbol{u}}=\operatorname{div} \boldsymbol{T}
$$

and the symmetry of $\boldsymbol{T}$.

Incompressibility of the fluid requires that the stress $\boldsymbol{T}$ admit a decomposition

$$
\boldsymbol{T}=-p \mathbf{1}+\mathbf{S}
$$

into a constitutively indeterminate pressure $p$ and an extra stress $\boldsymbol{S}(\operatorname{tr} \boldsymbol{S}=0)$ available for constitutive prescription.

\subsection{Bulk energy balance and entropy imbalance}

For $\mathscr{P}(t)$ a region that convects with the fluid, the first and second laws of thermodynamics, namely balance of energy and the entropy imbalance, have the respective forms

$$
\begin{aligned}
\frac{\mathrm{d}}{\mathrm{d} t} \int_{\mathscr{P}(t)} \varrho\left\{\varepsilon+\frac{1}{2}|\boldsymbol{u}|^{2}\right\} \mathrm{d} v & =\int_{\partial \mathscr{P}(t)} \boldsymbol{T} \boldsymbol{m} \cdot \boldsymbol{u} \mathrm{d} a-\int_{\partial \mathscr{P}(t)} \boldsymbol{q} \cdot \boldsymbol{m} \mathrm{d} a, \\
\frac{\mathrm{d}}{\mathrm{d} t} \int_{\mathscr{P}(t)} \varrho \eta \mathrm{d} v & \geqslant-\int_{\partial \mathscr{P}(t)} \frac{\boldsymbol{q}}{\vartheta} \cdot \boldsymbol{m} \mathrm{d} a,
\end{aligned}
$$

where $\varepsilon$ and $\eta$ denote the specific internal energy and specific entropy, while $\boldsymbol{q}$ is the heat flux and $\vartheta$ is the (absolute) temperature.

Using (2.2), (2.5), (2.7) and (2.8), we find that

$$
\varrho \dot{\varepsilon}=\boldsymbol{S}: \boldsymbol{D}-\operatorname{div} \boldsymbol{q}, \quad \varrho \dot{\eta} \geqslant-\operatorname{div} \frac{\boldsymbol{q}}{\vartheta},
$$

which are the local forms of the first two laws. If we introduce the specific free energy

$$
\psi=\varepsilon-\vartheta \eta,
$$

then, subtracting $(2.10 b)$ from $(2.10 a)$, we arrive at the local free-energy imbalance

$$
\varrho(\dot{\psi}+\eta \dot{\vartheta})-\boldsymbol{S}: \boldsymbol{D}+\frac{1}{\vartheta} \boldsymbol{q} \cdot \operatorname{grad} \vartheta \leqslant 0 .
$$

\subsection{Bulk constitutive relations}

Using the symbols \pm to label the phases, we take as bulk constitutive equations for the individual phases the classical state relations

$$
\psi=\psi^{ \pm}(\vartheta), \quad \eta=\eta^{ \pm}(\vartheta)=-\frac{\mathrm{d} \psi^{ \pm}(\vartheta)}{\mathrm{d} \vartheta},
$$

the auxiliary state relation

$$
\varepsilon=\varepsilon^{ \pm}(\vartheta)=\psi^{ \pm}(\vartheta)-\vartheta \frac{\mathrm{d} \psi^{ \pm}(\vartheta)}{\mathrm{d} \vartheta},
$$


which follows from (2.11) and (2.13), the viscous flow relation

$$
\boldsymbol{S}=2 \mu^{ \pm}(\vartheta) \boldsymbol{D}
$$

and Fourier's law

$$
\boldsymbol{q}=-k^{ \pm}(\vartheta) \operatorname{grad} \vartheta
$$

Here, the viscosities $\mu^{ \pm}$and conductivities $k^{ \pm}$, are strictly positive. For each phase, the constitutive relations (2.13)-(2.16) are consistent with the free-energy imbalance (2.12).

\section{Configurational forces in bulk}

When discussing configurational forces in solids, it is natural to employ a referential (or Lagrangian) description. Here, as we are discussing fluids, we recast in a spatial (or Eulerian) setting Gurtin's (1995, 2000) approach to configurational forces.

\subsection{Migrating control volumes. Observed and relative velocities}

To characterize the manner in which configurational forces expend power, a means of capturing the kinematics associated with the transfer of material is required. We accomplish this with the aid of control volumes $\mathscr{R}(t)$ that migrate relative to the fluid and thereby result in the transfer of material to - and the removal of material from $\mathscr{R}(t)$ at $\partial \mathscr{R}(t)$. Here, it is essential that regions $\mathscr{P}(t)$ convecting with the fluid not be confused with control volumes $\mathscr{R}(t)$ that migrate relative to the material.

Unless specified to the contrary, $\mathscr{R}(t)$ is a migrating control volume with $V_{\text {ә }}(\boldsymbol{x}, t)$ the (scalar) normal velocity of $\partial \mathscr{R}(t)$ in the direction of the outward unit normal $\boldsymbol{m}(\boldsymbol{x}, t)$. To describe power expenditures associated with the migration of $\mathscr{R}(t)$, we

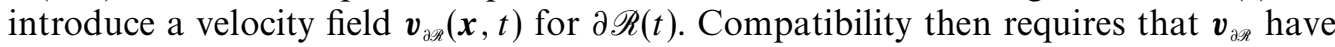
$V_{\text {ә }}$ as its normal component,

but $\boldsymbol{v}_{\partial \rtimes}$ is otherwise arbitrary.

$$
\boldsymbol{v}_{\partial \mathscr{X}} \cdot \boldsymbol{m}=V_{\partial \mathscr{X}},
$$

Non-normal velocity fields, while not intrinsic, are important. For example, given an arbitrary time-dependent parameterization $\boldsymbol{x}=\hat{\boldsymbol{x}}\left(\xi_{1}, \xi_{2}, t\right)$ of $\partial \mathscr{R}$, the field defined by $\boldsymbol{v}_{\partial \Re}=\partial \hat{\boldsymbol{x}} / \partial t$ (holding $\left(\xi_{1}, \xi_{2}\right)$ fixed), is a velocity field for $\partial \mathscr{R}$, but $\boldsymbol{v}_{2 \Re}$ is generally non-normal. We refer to the normal velocity $V_{\partial \curvearrowright}$ and any choice of the velocity field $\boldsymbol{v}_{\partial \mathscr{R}}$ for $\partial \mathscr{R}$ as observed velocities for $\partial \mathscr{R}$, since they represent velocity fields that characterize the motion of $\mathscr{R}$ through space, independent of the motion of the fluid. While it is important that we allow for the use of non-normal velocity fields, we require that the theory itself not depend on the particular observed velocity field used to describe a given migrating control volume.

We refer to this hypothesis, as intrinsicality. Intrinsicality is reminiscent of, but different from, the general requirement that physical theories be independent of the observer.

It is also possible to characterize the motion of $\mathscr{R}$ relative to the fluid; in this case, we use the migrational velocity and the normal migrational velocity

$$
\boldsymbol{v}_{\text {ə刃 }}-\boldsymbol{u}, \quad V_{\partial \Re}^{m i g}=V_{\text {ә囚 }}-\boldsymbol{u} \cdot \boldsymbol{m},
$$

as well as the field

$$
J_{\partial \Re}=\varrho V_{\partial \Re}^{m i g},
$$

which represents the migrational mass flow across $\partial \mathscr{R}$. 
Since $\operatorname{div} \boldsymbol{u}=0$, we may use (2.4) and (3.3) to conclude that

$$
\begin{aligned}
\frac{\mathrm{d}}{\mathrm{d} t} \int_{\mathscr{R}(t)} \varrho \Phi \mathrm{d} v & =\int_{\mathscr{R}(t)} \varrho \frac{\partial \Phi}{\partial t} \mathrm{~d} v+\int_{\partial \mathscr{R}(t)} \Phi \varrho V_{\partial \mathscr{R}} \mathrm{d} a \\
& =\int_{\mathscr{R}(t)} \varrho(\dot{\Phi}-\boldsymbol{u} \cdot \operatorname{grad} \Phi) \mathrm{d} v+\int_{\partial \mathscr{R}(t)} \Phi \varrho V_{\partial \mathscr{R}} \mathrm{d} a \\
& =\int_{\mathscr{R}(t)} \varrho \dot{\Phi} \mathrm{d} v+\int_{\partial \mathscr{R}(t)} \Phi J_{\partial \mathscr{R}} \mathrm{d} a .
\end{aligned}
$$

\subsection{Configurational momentum balance}

We begin by rewriting the standard momentum balances (2.6) in a form appropriate to a migrating control volume $\mathscr{R}(t)$ :

$$
\begin{aligned}
\frac{\mathrm{d}}{\mathrm{d} t} \int_{\mathscr{R}(t)} \varrho \boldsymbol{u} \mathrm{d} v & =\int_{\partial \mathscr{R}(t)}\left\{\boldsymbol{T} \boldsymbol{m}+\boldsymbol{u} J_{\partial \mathscr{R}}\right\} \mathrm{d} a, \\
\frac{\mathrm{d}}{\mathrm{d} t} \int_{\mathscr{R}(t)}(\boldsymbol{x}-\mathbf{0}) \times \varrho \boldsymbol{u} \mathrm{d} v & =\int_{\partial \mathscr{R}(t)}(\boldsymbol{x}-\mathbf{0}) \times\left\{\boldsymbol{T} \boldsymbol{m}+\boldsymbol{u} J_{\partial \mathscr{R}}\right\} \mathrm{d} a .
\end{aligned}
$$

To describe the configurational counterpart of these balances, we introduce three additional fields: a specific configurational momentum $\boldsymbol{p}$; a configurational stress $\boldsymbol{C}$; and an internal configurational body force $\boldsymbol{f}$. (Internal configurational forces are discussed in detail by Gurtin (2000, p. 10). In bulk; such forces are not so important, as they are indeterminate,) Then, guided by (3.5a), we posit a configurational momentum balance requiring that, for each migrating control volume $\mathscr{R}$

$$
\frac{\mathrm{d}}{\mathrm{d} t} \int_{\mathscr{R}(t)} \varrho \boldsymbol{p} \mathrm{d} v=\int_{\partial \mathscr{R}(t)}\left\{\boldsymbol{C} \boldsymbol{m}+\boldsymbol{p} J_{\partial \mathscr{R}}\right\} \mathrm{d} a+\int_{\mathscr{R}(t)} \boldsymbol{f} \mathrm{d} v .
$$

(We could also introduce an additional balance for moment of configurational momentum, but such a balance would be superfluous not only in bulk but, more importantly, in most situations involving a phase interface; cf. (5.12) of Gurtin (2000).) By (3.4), this balance has the local form

$$
\varrho \dot{\boldsymbol{p}}=\operatorname{div} \boldsymbol{C}+\boldsymbol{f} .
$$

In the balances (3.5) and (3.6), the vector fields $\boldsymbol{u} J_{\partial \gtrsim}$ and $\boldsymbol{p} J_{\partial \gtrsim}$ represent respective flows of linear and configurational momentum across $\partial \mathscr{R}$ induced by its migration. When there is no migration, that is when $J_{\partial \gtrsim}=0$ so that $\partial \mathscr{R}$ convects with the fluid, then these momentum flows vanish.

It is convenient to view the flows $\boldsymbol{u} J_{\partial \Re}$ and $\boldsymbol{p} J_{\partial \Re}$ as effective tractions associated with the flow of momentum across $\partial \mathscr{R}$, for then the momentum balances $(3.5 a)$ and (3.6) each has the form

$$
\frac{\mathrm{d}}{\mathrm{d} t}\{\text { momentum of } \mathscr{R}(t)\}=\{\text { effective net force on } \mathscr{R}(t)\} .
$$

This view is essential to our discussion of configurational forces.

\subsection{Configurational thermodynamics}

This section discusses forms of the energy balance and entropy imbalance appropriate to migrating control volumes. 
Standard form of the first and second laws

As is classical, the global forms of the energy balance and the entropy imbalance for a migrating control volume $\mathscr{R}(t)$ have the form

$$
\begin{aligned}
\frac{\mathrm{d}}{\mathrm{d} t} \int_{\mathscr{R}(t)} \varrho\left\{\varepsilon+\frac{1}{2}|\boldsymbol{u}|^{2}\right\} \mathrm{d} v-\int_{\partial \mathscr{R}(t)}\left\{\varepsilon+\frac{1}{2}|\boldsymbol{u}|^{2}\right\} J_{\partial \mathscr{R}} \mathrm{d} a & =\int_{\partial \mathscr{R}(t)} \boldsymbol{T} \boldsymbol{m} \cdot \boldsymbol{u} \mathrm{d} a-\int_{\partial \mathscr{R}(t)} \boldsymbol{q} \cdot \boldsymbol{m} \mathrm{d} a, \\
\frac{\mathrm{d}}{\mathrm{d} t} \int_{\mathscr{R}(t)} \varrho \eta \mathrm{d} v-\int_{\partial \mathscr{R}(t)} \eta J_{\partial \mathscr{R}} \mathrm{d} a & \geqslant-\int_{\partial \mathscr{R}(t)} \frac{\boldsymbol{q}}{\vartheta} \cdot \boldsymbol{m} \mathrm{d} a .
\end{aligned}
$$

In view of (3.3), the basic laws in this form account for the migration of $\mathscr{R}(t)$ through the underlined terms, which represent flows of energy and entropy across $\partial \mathscr{R}$. When $\mathscr{R}(t)$ convects with the fluid, $J_{\partial \mathscr{R}}=0$ and (3.9) reduce to (2.9).

Configurational form of the first two laws

For a migrating control volume, the first two laws in the standard form (3.9) account only implicitly for power expended by configurational forces. A thermodynamics better suited to the study of such forces may be based on the first two laws in a form

$$
\begin{aligned}
& \frac{\mathrm{d}}{\mathrm{d} t} \int_{\mathscr{R}(t)} \varrho\left\{\varepsilon+\frac{1}{2}|\boldsymbol{u}|^{2}\right\} \mathrm{d} v=\underbrace{\int_{\partial \mathscr{R}(t)}\left\{\left(\boldsymbol{T} \boldsymbol{m}+\boldsymbol{u} J_{\partial \mathscr{R}}\right) \cdot \boldsymbol{v}_{\partial \mathscr{R}}+\left(\boldsymbol{C m}+\boldsymbol{p} J_{\partial \mathscr{R}}\right) \cdot\left(\boldsymbol{v}_{\partial \mathscr{R}}-\boldsymbol{u}\right)\right\} \mathrm{d} a}_{W(\mathscr{R}(t))} \\
& +\underbrace{\int_{\partial \mathscr{R}(t)}\left\{\vartheta \eta J_{\partial \mathscr{R}}-\boldsymbol{q} \cdot \boldsymbol{m}\right\} \mathrm{d} a}_{Q(\mathscr{R}(t))}
\end{aligned}
$$

and

$$
\frac{\mathrm{d}}{\mathrm{d} t} \int_{\mathscr{R}(t)} \varrho \eta \mathrm{d} v \geqslant \underbrace{\int_{\partial \mathscr{R}(t)} \frac{\vartheta \eta J_{\partial \mathscr{R}}-\boldsymbol{q} \cdot \boldsymbol{m}}{\vartheta} \mathrm{d} a}_{H(\mathscr{R}(t))}
$$

that accounts explicitly for configurational power expenditures. Configurational forms of the first and second laws of thermodynamics were first given by Gurtin (1995) for situations where inertia is negligible. See also Gurtin (2000), whose discussion of configurational momentum is incorrect. Our treatment of inertia and the associated effective power expenditures is based on that of Cermelli \& Fried (1997). Before establishing the precise manner in which this form of the first two laws is equivalent to the standard form (3.9), we discuss the physical ideas underlying (3.10) and (3.11).

The abstract structure of, say, (3.10), namely

$$
\begin{aligned}
& \frac{\mathrm{d}}{\mathrm{d} t}\{\text { internal energy plus kinetic energy of } \mathscr{R}(t)\} \\
= & \underbrace{\{\text { effective power expended on } \mathscr{R}(t)\}}_{W(\mathscr{R}(t))}+\underbrace{\{\text { effective heat flow into } \mathscr{R}(t)\}}_{Q(\mathscr{R}(t))},
\end{aligned}
$$

treats a migrating control volume as a 'thermodynamic entity' in which the inflow of (internal plus kinetic) energy is subsumed by:

(i) an effective expenditure of power by standard forces, configurational forces and effective forces associated with the flow of momentum across surfaces;

(ii) an effective flow of heat. 
Note that this abstract structure is consistent with that of (3.8) for momentum.

The physical hypothesis underlying the form we choose for the effective power expenditure $W(\mathscr{R}(t))$ is the presumption that configurational forces expend power in

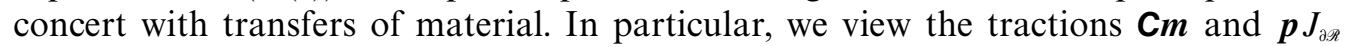
as forces associated with the transfer of material across $\partial \mathscr{R}$; since the migrational velocity $\boldsymbol{v}_{\text {ə冗 }}-\boldsymbol{u}$ represents the velocity with which material is transferred across $\partial \mathscr{R}$, we take $\boldsymbol{v}_{2 \Re}-\boldsymbol{u}$ to be an appropriate power-conjugate velocity for $\boldsymbol{C} \boldsymbol{m}+\boldsymbol{p} J_{\partial \Re}$; we therefore assume that the migration of $\mathscr{R}$ is accompanied by an effective power expenditure

$$
\int_{\partial \mathscr{R}(t)}\left\{\boldsymbol{C m}+\boldsymbol{p} J_{\partial \Re}\right\} \cdot\left(\boldsymbol{v}_{\partial \mathscr{R}}-\boldsymbol{u}\right) \mathrm{d} a .
$$

In deciding on the appropriate expenditure by standard tractions, it is important to emphasize that material is continually being transferred across $\partial \mathscr{R}$ as a result of its migration through the fluid; hence, $\partial \mathscr{R}$ has no intrinsic material description.

We therefore take the observed velocity $\boldsymbol{v}_{\partial \Re}$ of $\partial \mathscr{R}$, rather than the material velocity $\boldsymbol{u}$, as the appropriate conjugate velocity for $\boldsymbol{T} \boldsymbol{m}+\boldsymbol{u} J_{2 \Re}$, and write the effective standard power expenditure in the form

$$
\int_{\partial \mathscr{R}(t)}\left\{\boldsymbol{T} \boldsymbol{m}+\boldsymbol{u} J_{\partial \supsetneq}\right\} \cdot \boldsymbol{v}_{\partial \Re} \mathrm{d} a .
$$

Finally, the configurational body force $f$, being internal, is viewed as acting within the control volume $\partial \mathscr{R}$; as such $\boldsymbol{f}$ cannot affect the external power expenditure $W(\mathscr{R})$.

For the second law in the form (3.11) to be consistent with its standard form (3.9b), the entropy flow $\int_{\partial \mathscr{R}} \eta J_{\partial \mathscr{X}} \mathrm{d} a$ must join the standard entropy flow $-\int_{\partial \mathscr{R}}(\boldsymbol{q} \cdot \boldsymbol{m}) / \vartheta \mathrm{d} a$ to form the effective entropy flow $H(\mathscr{R})$ :

$$
H(\mathscr{R}(t))=\int_{\partial \mathscr{R}(t)} \frac{\vartheta \eta J_{\partial \mathscr{R}}-\boldsymbol{q} \cdot \boldsymbol{m}}{\vartheta} \mathrm{d} a .
$$

Further, if we require that, on $\partial \mathscr{R}$,

$$
\text { entropy flow per unit area }=\frac{\text { heat flow per unit area }}{\vartheta},
$$

then we are led to consider

$$
Q(\mathscr{R}(t))=\int_{\partial \mathscr{R}(t)}\left\{\vartheta \eta J_{\partial \mathscr{R}}-\boldsymbol{q} \cdot \boldsymbol{m}\right\} \mathrm{d} a
$$

as the appropriate choice for the effective heat flow across $\partial \mathscr{R}$. We therefore view $\vartheta \eta J_{\partial \varkappa}$ and $\eta J_{\partial \circledast}$ as respective flows of heat and entropy induced by the migration of $\partial \mathscr{R}$.

Equivalence of the standard and configurational forms of the first two laws

We now turn to a rigorous justification of our use of the configurational forms of the first two laws. First of all, the configurational and standard forms, (3.11) and (3.9b), of the second law are clearly equivalent, and the former trivially satisfies intrinsicality. We may therefore limit our discussion to the first law. Regarding this law in the configurational form (3.10), note that the intrinsicality hypothesis is equivalent to the requirement that the power $W(\mathscr{R}(t))$ be independent of the choice of observed velocity field $\boldsymbol{v}_{\partial \Re}$ chosen to characterize the migration of $\mathscr{R}(t)$.

EQuivalenCy THeOREM. The first law in the configurational form (3.10), subject to the intrinsicality hypothesis, is equivalent to the first law in the standard form (3.9a), 
supplemented by the Eshelby relation

$$
\boldsymbol{C}=\varrho\left\{\psi-\frac{1}{2}|\boldsymbol{u}|^{2}\right\} \mathbf{1}-\boldsymbol{T}
$$

and the momentum relation

$$
\boldsymbol{p}=-\boldsymbol{u}
$$

Proof. Consider the first law in the configurational form (3.10). Our first step is to determine the consequences of the invariance requirement. Since all observed velocity fields have the same normal component, while the tangential components are arbitrary, the invariance requirement means that $W(\mathscr{R}(t))$ be invariant under all transformations of the form

$$
\boldsymbol{v}_{\text {ə刃 }} \mapsto \boldsymbol{v}_{\partial \Re}+\xi \boldsymbol{t},
$$

with $\xi$ an arbitrary scalar field and $\boldsymbol{t}$ with $|\boldsymbol{t}|=1$ a tangential vector field on $\partial \mathscr{R}$, or, equivalently, by (3.3), that

$$
\int_{\partial \mathscr{R}(t)} \xi \boldsymbol{t} \cdot\left\{(\boldsymbol{T}+\boldsymbol{C}) \boldsymbol{m}+\varrho(\boldsymbol{u}+\boldsymbol{p}) V_{\partial \mathscr{R}}^{m i g}\right\} \mathrm{d} a=0
$$

for all such transformations. Thus, since $\mathscr{R}, \xi$, and $t$ (tangential to $\partial \mathscr{R}$ ) may be arbitrarily chosen, it follows that

$$
\boldsymbol{t} \cdot\left\{(\boldsymbol{T}+\boldsymbol{C}) \boldsymbol{m}+\varrho(\boldsymbol{u}+\boldsymbol{p}) V_{\partial \boldsymbol{z}}^{m i g}\right\}=0
$$

for any scalar field $V_{\partial \Re}^{m i g}$ and all unit vectors $\boldsymbol{t}$ and $\boldsymbol{m}$ with $\boldsymbol{t}$ orthogonal to $\boldsymbol{m}$. Since $V_{\partial \Re}^{m i g}$ is arbitrary, $\boldsymbol{t} \cdot(\boldsymbol{u}+\boldsymbol{p})=0$ for all unit vectors $\boldsymbol{t}$, so that $\boldsymbol{p}=-\boldsymbol{u}$, which is (3.17). Thus, letting $\boldsymbol{A}=\boldsymbol{T}+\boldsymbol{C}$, it follows that, for each $\boldsymbol{m}, \boldsymbol{A} \boldsymbol{m}$ must lie in the direction of $\boldsymbol{m}$, which is possible if and only if $\boldsymbol{A}$ has the form $\boldsymbol{A}=\varrho \varphi \mathbf{1}$, with $\varphi$ a scalar field. Invariance therefore yields the pre-Eshelby relation

$$
\boldsymbol{C}=\varrho \varphi \mathbf{1}-\boldsymbol{T} .
$$

In view of (3.17) and (3.19), the power expended on $\mathscr{R}$ becomes

$$
W(\mathscr{R}(t))=\int_{\partial \mathscr{R}(t)}\left\{\boldsymbol{T} \boldsymbol{m} \cdot \boldsymbol{u}+\left\{\varphi+|\boldsymbol{u}|^{2}\right\} \boldsymbol{J}_{\partial \mathscr{R}}\right\} \mathrm{d} a .
$$

Further, since $\psi=\varepsilon-\vartheta \eta$, we may use (3.4) and (3.20) to write (3.10) in the form:

$$
\int_{\mathscr{R}(t)} \varrho \overline{\left\{\varepsilon+\frac{1}{2}|\boldsymbol{u}|^{2}\right\}} \mathrm{d} v=\int_{\partial \mathscr{R}(t)} \boldsymbol{T} \boldsymbol{m} \cdot \boldsymbol{u} \mathrm{d} a-\int_{\partial \mathscr{R}(t)} \boldsymbol{q} \cdot \boldsymbol{m} \mathrm{d} a+\int_{\partial \mathscr{R}(t)}\left\{\varphi+\frac{1}{2}|\boldsymbol{u}|^{2}-\psi\right\} \boldsymbol{J}_{\partial \mathscr{R}} \mathrm{d} a .
$$

At this point, it is important to note that, by (3.2) and (3.3), the sole term in (3.21) involving the normal velocity $V_{\partial \Re}$ is the term with integrand $\left(\varphi+\frac{1}{2}|\boldsymbol{u}|^{2}-\psi\right) J_{\text {ə⿻ }}=$ $\varrho\left(\varphi+\frac{1}{2}|\boldsymbol{u}|^{2}-\psi\right)\left(V_{\partial \mathscr{R}}-\boldsymbol{u} \cdot \boldsymbol{m}\right)$. Bearing this in mind, we note that, given any time $t_{0}$, we may choose a second migrating control volume that coincides with $\mathscr{R}(t)$ at $t=t_{0}$, but for which $V_{\partial \mathscr{R}}$ at $t_{0}$ is arbitrary. Thus, for (3.21) to hold for all migrating control volumes, we must have

$$
\varphi=\psi-\frac{1}{2}|\boldsymbol{u}|^{2}
$$

therefore, by (3.19), the Eshelby relation (3.16) holds. Further, by (3.22), we may use (3.4) to reduce (3.21) to the standard form (3.9a) of the first law. We have therefore shown that the configurational form of the first law and the intrinsicality hypothesis together imply both the standard form of this law and the Eshelby relation. 
To prove the converse assertion, assume that both the standard form (3.9a) of the first law, the Eshelby relation (3.16), and the momentum relation (3.17) are satisfied. Choose an arbitrary velocity field $\boldsymbol{v}_{\partial \Re}$ for $\partial \mathscr{R}$ and note that, since $\boldsymbol{v}_{\partial \Re} \cdot \boldsymbol{m}=V_{\partial \mathscr{\Re}}$ and $J_{\partial \gtrsim}=\varrho\left(V_{\text {ә }}-\boldsymbol{u} \cdot \boldsymbol{m}\right)$, it follows, upon appealing to the Eshelby relation (3.16), that

$$
\begin{aligned}
& T m \cdot u=T m \cdot v_{\text {ə刃 }}-T m \cdot\left(v_{2 \Re}-u\right)
\end{aligned}
$$

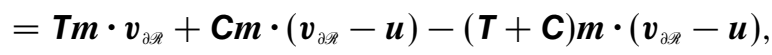

$$
\begin{aligned}
& =\boldsymbol{T} \boldsymbol{m} \cdot \boldsymbol{v}_{\text {əれ }}+\mathbf{C m} \cdot\left(\boldsymbol{v}_{2 \Re}-\boldsymbol{u}\right)-\left\{\psi-\frac{1}{2}|\boldsymbol{u}|^{2}\right\} \boldsymbol{J}_{2 \Re} .
\end{aligned}
$$

Further, since $\boldsymbol{p}=-\boldsymbol{u}$, it follows that $|\boldsymbol{u}|^{2}=\boldsymbol{u} \cdot \boldsymbol{v}_{\text {ə刃 }}+\boldsymbol{p} \cdot\left(\boldsymbol{v}_{2 刃}-\boldsymbol{u}\right)$, and this equation and (3.23) imply that

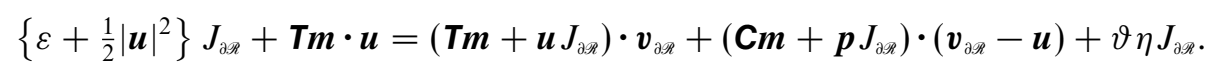

This identity reduces the first law in the standard form (3.9a) to its configurational counterpart (3.10). The proof of the equivalency theorem is now complete.

We have shown that, granted intrinsicality, the configurational and standard forms of the first two laws are equivalent. For that reason, our discussion of configurational forces in bulk might seem superfluous, but it is not; this discussion provides a vehicle for introducing the basic structure and central ideas, these are not at all transparent when discussing phase interfaces. In fact, our treatment of phase interfaces is based on thermomechanical laws that are direct counterparts of the configurational laws (3.10) and (3.11) and that lead, via intrinsicality, to an interfacial Eshelby relation analogous to the bulk Eshelby relation (3.16).

\subsection{Specific form of the configurational fields}

Until this stage in our discussion of configurational forces, no use has been made of constitutive theory. Our results, being independent of constitutive equations, therefore apply to broad classes of materials. Moreover, since the standard force balance (2.7) requires that $\varrho \dot{\boldsymbol{u}}=\operatorname{div} \boldsymbol{T}$, the Eshelby and momentum relations (3.16) and (3.17) yield, for each of the two phases, the relation $\operatorname{div} \boldsymbol{C}-\varrho \dot{\boldsymbol{p}}=\varrho \operatorname{grad}\left\{\psi-|\boldsymbol{u}|^{2} / 2\right\}$, where we have used the assumed constancy of $\varrho$ in each phase. This result, which is independent of constitution, shows that the internal body force $f$ in the configurational balance (3.7) has the explicit form

$$
\boldsymbol{f}=-\varrho \operatorname{grad}\left\{\psi-\frac{1}{2}|\boldsymbol{u}|^{2}\right\} .
$$

Thus, granted (3.24) and the Eshelby relation, the configurational force balance in bulk is a direct consequence of the standard force balance. On the other hand, as we shall see, the interfacial configurational momentum balance is an independent balance, not derivable from standard interfacial results (cf. the materials literature, where interfacial configurational balances are often determined via a variational principle, assuming equilibrium, and then used as missing interface conditions for dynamical problems).

As a consequence of (3.24), we see that the internal configurational force $f$ arises as a response to an inhomogeneous distribution of free and kinetic energy (and hence because the free energy depends constitutively on the temperature - as a response to inhomogeneous temperature and velocity fields). Thus, roughly speaking, internal configurational forces characterize microphysical forces that act within the material as a response to energetic inhomogeneities. This need for internal configurational forces becomes primal when discussing sharp phase interfaces: the interface in a two-phase system generally represents a surface of discontinuity (and hence inhomogeneity) of the free and kinetic energies. 
Using (3.24), the Eshelby relation (3.16), the momentum relation (3.17), and the bulk constitutive relations, we find that the configurational stress $\boldsymbol{C}^{ \pm}$and internal configurational force $\boldsymbol{f}^{ \pm}$in the individual bulk phases are determined by the fields $\boldsymbol{u}$, $\vartheta$ and $p$. As we shall see, interfacial counterparts of $\boldsymbol{C}^{+}$and $\boldsymbol{f}^{+}$, which we view as surface excess quantities, do require independent constitutive specification.

\section{Interfacial kinematics}

\subsection{The interface $\mathscr{S}$. Superficial fields}

We assume that the interface $\mathscr{S}(t)$ separating the phases is a smoothly evolving surface oriented by a unit normal field $\boldsymbol{n}(\boldsymbol{x}, t)$. We write $V(\boldsymbol{x}, t)$ for the (scalar) normal velocity of $\mathscr{S}(t)$.

A superficial field is a smooth field defined on the interface for all time. A superficial vector field $\boldsymbol{g}$ is tangential if $\boldsymbol{g} \cdot \boldsymbol{n}=0$. For a superficial tensor field $\mathbb{B}$ we require that

$$
\mathbb{B} \boldsymbol{n}=\mathbf{0} \text {; }
$$

if, in addition, $\mathbb{B}^{\top} \boldsymbol{n}=\mathbf{0}$, then we say that $\mathbb{B}$ is fully tangential, so that $\mathbb{B}$ maps tangent vectors to tangent vectors. An example of a fully tangential tensor field is the projection

$$
\mathbb{P}=\mathbf{1}-\boldsymbol{n} \otimes \boldsymbol{n}
$$

onto $\mathscr{S}$; clearly,

$$
\mathbb{P}: \mathbb{P}=2 .
$$

(A superficial tensor field would generally be defined at each $\boldsymbol{x} \in \mathscr{S}$ as a linear transformation of the tangent space at $\boldsymbol{x}$ into $\mathbb{R}^{3}$; the requirement (4.1) allows us to consider $\mathbb{B}$ at each point as a linear transformation of $\mathbb{R}^{3}$ into $\mathbb{R}^{3}$.)

Each superficial tensor field $\mathbb{B}$ admits a decomposition of the form

$$
\mathbb{B}=\mathbb{B}_{\tan }+\boldsymbol{n} \otimes \boldsymbol{b},
$$

in which $\mathbb{B}_{\tan }=\mathbb{P B}$ is fully tangential and $\boldsymbol{b}=\mathbb{B}^{\top} \boldsymbol{n}$ is tangential. The verification of this decomposition is straightforward: simply expand $\mathbb{P B}$ using (4.2).

\subsection{Surface gradient and divergence}

We write $\operatorname{grad}_{\mathscr{S}}$ and $\operatorname{div}_{\mathscr{S}}$ for the surface gradient and surface divergence on $\mathscr{S}$.

The curvature tensor $\mathbb{K}$ defined by

$$
\mathbb{K}=-\operatorname{grad}_{s} n
$$

is fully tangential and symmetric, and

$$
K=\operatorname{tr} \mathbb{K}=\mathbb{P}: \mathbb{K}=-\operatorname{div}_{\mathscr{S}} \boldsymbol{n}
$$

is the total (twice the mean) curvature. Then, by (4.2), we have the identity

$$
\operatorname{div}_{\mathscr{S}} \mathbb{P}=K \boldsymbol{n} .
$$

Further, the product rule yields the useful identities

$$
\operatorname{grad}_{\mathscr{S}}(V \boldsymbol{n})=\boldsymbol{n} \otimes \operatorname{grad}_{\mathscr{S}} V-V \mathbb{K}, \quad \operatorname{div}_{\mathscr{S}}(V \boldsymbol{n})=-K V,
$$

involving the interfacial normal, normal velocity and curvature.

Let $\mathbb{B}$ be a fully tangential tensor field. Then

$$
\boldsymbol{n} \cdot \operatorname{div}_{\mathscr{S}} \mathbb{B}=\operatorname{div}_{\mathscr{S}}(\underbrace{\mathbb{B}^{\top} \boldsymbol{n}}_{=\mathbf{0}})-\mathbb{B}: \operatorname{grad}_{\mathscr{S}} \boldsymbol{n},
$$


and (4.5) yields the important identity

$$
\boldsymbol{n} \cdot \operatorname{div}_{s} \mathbb{B}=\mathbb{B}: \mathbb{K}, \quad \text { for } \quad \mathbb{B} \text { fully tangential. }
$$

We assume that each bulk field is smooth up to the interface from either side. We write $\llbracket \Phi \rrbracket$ and $\langle\langle\Phi\rangle$ for the jump and average of a bulk field $\Phi$ across $\mathscr{S}: \llbracket \Phi \rrbracket$ is the interfacial limit of $\Phi$ from the (+) phase (the phase into which $\boldsymbol{n}$ points) minus its limit from the $(-)$ phase; $\langle\langle\Phi\rangle$ is the average value of these two limits. Then, given bulk fields $\Phi$ and $\Lambda$, we have the important identity:

$$
\llbracket \Phi \Lambda \rrbracket=\langle\langle\Phi\rangle \llbracket \Lambda \rrbracket+\langle\langle\Lambda\rangle \llbracket \Phi \rrbracket .
$$

\subsection{Mass balance. Mass flow across the interface}

The fields

$$
\varrho^{ \pm}\left(V-\boldsymbol{u}^{ \pm} \cdot \boldsymbol{n}\right)
$$

represent flows of mass at the two sides of the interface in the direction $\boldsymbol{n}$, and balance of mass requires that these be equal:

$$
\llbracket \varrho(V-\boldsymbol{u} \cdot \boldsymbol{n}) \rrbracket=0 .
$$

Thus, we may define the mass flow $J$ across the interface (in the direction $\boldsymbol{n}$ ) by the relation $J=\varrho^{ \pm}\left(V-\boldsymbol{u}^{ \pm} \cdot \boldsymbol{n}\right)$, so that

$$
J=\langle u\rangle^{-1} V^{m i g}, \quad \llbracket v \rrbracket J=-\llbracket u \rrbracket \cdot \boldsymbol{n},
$$

with

$$
V^{m i g} \stackrel{\text { def }}{=} V-\langle\langle\boldsymbol{u}\rangle\rangle \cdot \boldsymbol{n}
$$

the average migrational velocity of the interface relative to the bulk material.

\subsection{Smoothness of $\boldsymbol{u}$ at the interface}

We allow for the possibility that the densities of the phases differ and therefore, by (4.12), we must allow for the possibility that the normal components $\boldsymbol{u}^{ \pm} \cdot \boldsymbol{n}$ of the interfacial limits $\boldsymbol{u}^{ \pm}$of $\boldsymbol{u}$ differ. On the other hand, we assume that

$$
\mathbb{P} \llbracket u \rrbracket=0,
$$

so that the tangential components $\mathbb{P} \boldsymbol{u}^{ \pm}$of the interfacial limits $\boldsymbol{u}^{ \pm}$of $\boldsymbol{u}$ coincide. In addition, we assume that $\boldsymbol{u}$ is smooth up to the interface from either side.

We write

$$
\left.\mathbb{L}=\operatorname{grad}_{\mathscr{S}}\langle\boldsymbol{u}\rangle\right\rangle=\langle\langle\boldsymbol{L}\rangle \mathbb{P}=\langle\boldsymbol{D}+\boldsymbol{W}\rangle \mathbb{P}
$$

for the superficial velocity gradient and

$$
\left.\mathbb{D}=\frac{1}{2}\left\{\mathbb{P} \mathbb{L}+\mathbb{L}^{\top} \mathbb{P}\right\}=\mathbb{P}\langle\boldsymbol{D}\rangle\right\rangle \mathbb{P}
$$

for the superficial rate of stretch. Then, for $\mathbb{B}$ a tangential and symmetric superficial tensor field,

$$
\mathbb{B}: \mathbb{L}=\mathbb{B}: \mathbb{D} .
$$

Further, since $\mathbb{D}$ is tangential,

$$
\mathbb{P}: \mathbb{D}=\operatorname{tr} \mathbb{D} .
$$

\subsection{Velocity fields}

We let $\boldsymbol{v}(\boldsymbol{x}, t)$ denote a velocity field for $\mathscr{S}$; that is, a velocity field describing the evolution of $\mathscr{S}$. Then the normal component of $v$ must satisfy

$$
V=\boldsymbol{v} \cdot \boldsymbol{n},
$$


but the tangential part, $\mathbb{P} \boldsymbol{v}$, which is not intrinsic, may be arbitrarily chosen. The fields

$$
\boldsymbol{v}-\boldsymbol{u}^{ \pm}, \quad V-\boldsymbol{u}^{ \pm} \cdot \boldsymbol{n}
$$

represent migrational velocites of the interface relative to the material in each of the bulk phases.

Consider an arbitrary migrating subsurface $\mathscr{A}(t)$ of $\mathscr{S}(t)$. To describe the migration of $\mathscr{A}(t)$, we introduce a field $\boldsymbol{v}_{\partial \mathscr{A}}(\boldsymbol{x}, t)$ defined over $\partial \mathscr{A}(t)$ for all $t$. Compatibility then requires that

$$
\boldsymbol{v}_{\partial \mathscr{A}} \cdot \boldsymbol{n}=V, \quad \boldsymbol{v}_{\partial \mathscr{A}} \cdot \boldsymbol{v}_{\partial \mathscr{A}}=V_{\partial \mathscr{A}},
$$

where $V_{\partial \mathscr{A}}$, which is intrinsic, is the scalar normal velocity of $\partial \mathscr{A}$ in the direction of its normal $\boldsymbol{v}_{\partial \mathscr{A}}$.

The motion of $\partial \mathscr{A}$ relative to the bulk material is described by the migrational velocities $\boldsymbol{v}_{\partial \mathscr{A}}-\boldsymbol{u}^{ \pm}$and average migrational velocity $\boldsymbol{v}_{\partial \mathscr{A}}-\langle\langle\boldsymbol{u}\rangle$. Further, bearing in mind that $\boldsymbol{u}^{+} \cdot \boldsymbol{v}_{\partial \mathscr{A}}=\boldsymbol{u}^{-} \cdot \boldsymbol{v}_{\partial \mathscr{A}}$,

$$
V_{\partial \mathscr{A}}^{m i g} \stackrel{\text { def }}{=} V_{\partial \mathscr{A}}-\boldsymbol{u}_{t a n} \cdot \boldsymbol{v}_{\partial \mathscr{A}}=V_{\partial \mathscr{A}}-\left\langle\langle\boldsymbol{u}\rangle \cdot \boldsymbol{v}_{\partial \mathscr{L}}=V_{\partial \mathscr{L}}-\boldsymbol{u}^{ \pm} \cdot \boldsymbol{v}_{\partial \mathscr{A}}\right.
$$

represents the normal migrational velocity of $\partial \mathscr{A}$.

The component of $\boldsymbol{v}_{\partial \mathscr{A}}$ tangential to $\partial \mathscr{A}$ is not intrinsic and may be arbitrarily chosen. We require that the theory not depend on the velocity field $\boldsymbol{v}_{\partial \mathscr{A}}$ chosen to characterize the migration of $\partial \mathscr{A}$, and therefore that the theory be invariant under transformations of $\boldsymbol{v}_{\partial \mathscr{A}}$ of the form

$$
\boldsymbol{v}_{\partial \mathscr{A}} \rightarrow \boldsymbol{v}_{\partial \mathscr{A}}+\boldsymbol{t}, \quad \boldsymbol{t} \text { tangent to } \partial \mathscr{A} .
$$

\subsection{Migrationally normal velocity field for $\mathscr{S}$}

In discussing the formulation of integral balance laws for an interface $\mathscr{S}(t)$ migrating through the fluid, what is required is a velocity field for $\mathscr{S}$ that characterizes its migration. Specifically, we seek a single velocity field $\boldsymbol{v}$ for $\mathscr{S}$ that renders each of the migrational velocities $\boldsymbol{v}-\boldsymbol{u}^{ \pm}$normal. With this in mind, we let $\boldsymbol{v}_{\text {tan }}$ and $\boldsymbol{u}_{\text {tan }}$, respectively, denote the tangential component of $\boldsymbol{v}$ and the common tangential component of $\boldsymbol{u}^{+}$and $\boldsymbol{u}^{-}$,

$$
\boldsymbol{v}_{\text {tan }} \stackrel{\text { def }}{=} \mathbb{P} \boldsymbol{v}, \quad \boldsymbol{u}_{\text {tan }} \stackrel{\text { def }}{=} \mathbb{P} \boldsymbol{u}^{+}=\mathbb{P} \boldsymbol{u}^{-},
$$

and note that

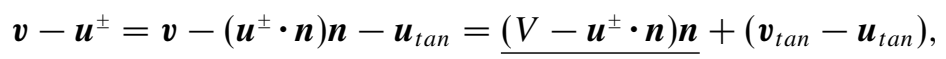

so that, taking $\boldsymbol{v}_{\text {tan }}=\boldsymbol{u}_{\text {tan }}$, we arrive at a choice of velocity field $\boldsymbol{v}$ for $\mathscr{S}$ with each of its migrational velocities $\boldsymbol{v}-\boldsymbol{u}^{ \pm}$normal:

$$
\boldsymbol{v}-\boldsymbol{u}^{ \pm}=\left(V-\boldsymbol{u}^{ \pm} \cdot \boldsymbol{n}\right) \boldsymbol{n} .
$$

Since $\boldsymbol{u}^{ \pm}-\left(\boldsymbol{u}^{ \pm} \cdot \boldsymbol{n}\right) \boldsymbol{n}=\boldsymbol{u}_{\text {tan }}$, the resulting velocity field $\boldsymbol{v}$, called the migrationally normal velocity field for $\mathscr{S}$, has the specific form

$$
\boldsymbol{v}=V \boldsymbol{n}+\boldsymbol{u}_{\text {tan }}
$$

and is important because it is normal when computed relative to the material on either side of $\mathscr{S}(t)$.

In view of (4.14), for $v$ the migrationally normal velocity field for $\mathscr{S}$, the migrational velocity $\boldsymbol{v}-\langle\langle\boldsymbol{u}\rangle\rangle$ satisfies

$$
\boldsymbol{v}-\left\langle\langle\boldsymbol{u}\rangle=V^{m i g} \boldsymbol{n}\right.
$$




$$
\operatorname{grad}_{\mathscr{S}}(\boldsymbol{v}-\langle\boldsymbol{u}\rangle)=\boldsymbol{n} \otimes \operatorname{grad}_{\mathscr{S}} V^{m i g}-V^{m i g} \mathbf{K},
$$

Further, by (4.22), (4.23) and (4.26), we also have the identity

$$
\left(\boldsymbol{v}_{\partial \alpha}-\boldsymbol{v}\right) \cdot \boldsymbol{v}_{\partial \alpha}=\left(\boldsymbol{v}_{\partial \alpha}-\boldsymbol{u}^{ \pm}\right) \cdot \boldsymbol{v}_{\partial \alpha}-\overbrace{\left(\boldsymbol{v}-\boldsymbol{u}^{ \pm}\right) \cdot \boldsymbol{v}_{\partial \alpha}}^{=0}=V_{\partial \alpha}^{m i g} .
$$

\subsection{Migrationally normal time derivative following $\mathscr{S}$}

Defining a meaningful time derivative of, say, a superficial scalar field $\gamma(\boldsymbol{x}, t)$ is a non-trivial matter: note that the standard partial derivative $\partial \gamma / \partial t$ is not well defined, because a point $\boldsymbol{x}$ on $\mathscr{S}\left(t_{0}\right)$ need not lie on $\mathscr{S}(t)$ for $t$ close to $t_{0}$. For our purposes, what is required is a time derivative that, in some sense, follows the migration of the interface.

With this in mind, let $\boldsymbol{v}$ denote a velocity field for $\mathscr{S}$. Then given any time $t_{0}$ and any point $\boldsymbol{x}_{0}$ on $\mathscr{S}\left(t_{0}\right)$, the solution $\boldsymbol{z}(t)$ of

$$
\frac{\mathrm{d} z(t)}{\mathrm{d} t}=\boldsymbol{v}(z(t), t), \quad z\left(t_{0}\right)=\boldsymbol{x}_{0}
$$

represents the trajectory of a 'particle' that, at each time, lies on $\mathscr{S}$ and and has velocity $\boldsymbol{v}$. In particular, let $\boldsymbol{v}$ denote the migrationally normal velocity field for $\mathscr{S}$ (cf. (4.27)) and let

$$
\left.\stackrel{\circ}{\gamma}\left(x_{0}, t_{0}\right) \stackrel{\text { def }}{=} \frac{\mathrm{d} \gamma(z(t), t)}{\mathrm{d} t}\right|_{t=t_{0}} ;
$$

the field $\dot{\gamma}$ defined in this manner is referred to as the migrationally normal time derivative of $\gamma$ following $\mathscr{S}$. (This notion as well as that of a migrationally normal velocity field are introduced by Cermelli, Fried \& Gurtin (2005), who show that ${ }^{\circ}$ is related to the (standard) normal time derivative $\gamma$ following $\mathscr{S}$ through the relation $\dot{\gamma}=\vec{\gamma}+\boldsymbol{u}_{\text {tan }} \cdot \operatorname{grad}_{\mathscr{\gamma}} \gamma ; \dot{\gamma}$ is based on the use of normal trajectories determined via (4.30) using the vector normal-velocity $\boldsymbol{v}=V \boldsymbol{n}$.)

\subsection{Two-phase migrating control volumes}

Let $\mathscr{R}(t)$ denote an arbitrary two-phase migrating control volume; that is, a migrating control volume whose interior intersects the interface, so that the portion of $\mathscr{S}(t)$ in $\mathscr{R}(t)$,

$$
\mathscr{A}(t)=\mathscr{R}(t) \cap \mathscr{S}(t),
$$

is a smoothly evolving subsurface of $\mathscr{S}(t)$. Let $\mathscr{R}^{+}(t)$ and $\mathscr{R}^{-}(t)$ be the portions of $\mathscr{R}(t)$ that lie in the (+) and (-) phases, respectively, so that, for $\boldsymbol{m}$ the outward unit normal to $\partial \mathscr{R}(t)$ :

(i) $\mathscr{R}^{+}(t)$ and $\mathscr{R}^{-}(t)$ are migrating control volumes;

(ii) the boundary $\partial \mathscr{R}^{ \pm}(t)$ of $\mathscr{R}^{ \pm}(t)$ is the union of $\mathscr{A}(t)$ and a surface $\partial \mathscr{R}_{b l k}^{ \pm}(t)$ that does not intersect the interface;

(iii) the outward unit normals to $\partial \mathscr{R}^{+}$and $\partial \mathscr{R}^{-}$are

$$
\left.\boldsymbol{m}\right|_{\partial \mathscr{R}^{+}}=\left\{\left.\begin{array}{lll}
\boldsymbol{m} & \text { on } & \partial \mathscr{R}_{b l k}^{+}, \\
-\boldsymbol{n} & \text { on } & \mathscr{A},
\end{array} \quad \boldsymbol{m}\right|_{\partial \mathscr{R}^{-}}=\left\{\begin{array}{lll}
\boldsymbol{m} & \text { on } & \partial \mathscr{R}_{b l k}^{-}, \\
\boldsymbol{n} & \text { on } & \mathscr{A} ;
\end{array}\right.\right.
$$

(iv) given a velocity field $\boldsymbol{v}_{2 \Re}$ for $\partial \mathscr{R}$, the associated velocity fields for $\partial \mathscr{R}^{+}$and $\partial \mathscr{R}^{-}$ are given by

$$
\boldsymbol{v}_{\partial \mathscr{A}^{+}}=\left\{\begin{array}{lll}
\boldsymbol{v}_{\partial \Re} & \text { on } & \partial \mathscr{R}_{b l k}^{+}, \\
\boldsymbol{v} & \text { on } & \mathscr{A},
\end{array} \quad \boldsymbol{v}_{\partial \mathscr{A}^{-}}=\left\{\begin{array}{lll}
\boldsymbol{v}_{\partial \Re} & \text { on } & \partial \mathscr{R}_{b l k}^{-} \\
\boldsymbol{v} & \text { on } & \mathscr{A}
\end{array}\right.\right.
$$


Note that

$$
\mathscr{R}(t)=\mathscr{R}^{+}(t) \cup \mathscr{R}^{-}(t)
$$

represents a decomposition of $\mathscr{R}(t)$ into single-phase migrating control volumes $\mathscr{R}^{+}(t)$ and $\mathscr{R}^{-}(t)$, because $\mathscr{R}^{+}(t)$ contains only plus-phase material, and similarly for $\mathscr{R}^{-}(t)$. The theory for the interface is based on two-phase migrating control volumes $\mathscr{R}(t)$ and - because the intersection of $\partial \mathscr{R}$ with $\mathscr{A}$ is a set of zero area - involves integrals $\int_{\partial \mathscr{R}} \ldots \mathrm{d} a$ no different from their bulk-theoretic conterparts. Our localization of such integrals to the interface is based on decompositions of the form

$$
\int_{\partial \mathscr{R}} \cdots \mathrm{d} a=\int_{\partial \mathscr{R}^{-}} \cdots \mathrm{d} a+\int_{\partial_{\mathscr{R}^{+}}} \cdots \mathrm{d} a+\int_{\mathscr{A}^{-}} \cdots \mathrm{d} a .
$$

We refer to any such decomposition as the distillation of $\int_{\partial \mathscr{R}} \cdots \mathrm{d} a$, because $\mathscr{R}^{+}$and $\mathscr{R}^{-}$contain only single-phase bulk material. Such distillations allow for the application of bulk results to eliminate terms of the form $\int_{\partial \mathscr{R} \pm} \cdots \mathrm{d} a$ in any given balance (or imbalance), thereby reducing each term of the form $\int_{\partial \mathscr{R}} \cdots \mathrm{d} a$ to an interfacial term $\int_{\mathscr{A}} \cdots \mathrm{d} a$.

\section{Interfacial mechanics}

In this section, we introduce basic balances for standard and configurational forces that account for forces within the interface. We also discuss the power expenditures associated with the effective standard and configurational surface tractions on a twophase migrating control volume. The localization of the standard and configurational force balances to points on the interface uses a tensorial form of the surface divergence theorem. Further, to develop an intrinsic expression for the power, we use the surface divergence theorem in yet another form.

Surface Divergence Theorem. Let $\mathbb{B}$ be a superficial tensor field, and let $\boldsymbol{h}$ be a superficial vector field. Then the surface divergence theorem asserts that, for any subsurface $\mathscr{A}$ of $\mathscr{S}$,

$$
\begin{gathered}
\int_{\partial \mathscr{A}} \mathbb{B} \boldsymbol{v}_{\partial \mathscr{A}} \mathrm{d} s=\int_{\mathscr{A}} \operatorname{div}_{\mathscr{f}} \mathbb{B} \mathrm{d} a, \\
\int_{\partial \mathscr{A}} \mathbf{B} \boldsymbol{v}_{\partial \mathscr{A}} \cdot \boldsymbol{h} \mathrm{d} s=\int_{\mathscr{A}}\left\{\boldsymbol{h} \cdot \operatorname{div}_{\mathscr{S}} \mathbb{B}+\mathbb{B}: \operatorname{grad}_{\mathscr{S}} \boldsymbol{h}\right\} \mathrm{d} a .
\end{gathered}
$$

These forms of the surface divergence theorem are based on the following more standard form in which $\boldsymbol{g}$ is a tangential superficial vector field:

$$
\int_{\partial \mathscr{A}} \boldsymbol{g} \cdot \boldsymbol{v}_{\partial \mathscr{A}} \mathrm{d} s=\int_{\mathscr{A}} \operatorname{div}_{\mathscr{S}} \boldsymbol{g} \mathrm{d} a .
$$

To derive the relations in (5.1), we simply let $\boldsymbol{a}$ be an arbitrary constant vector and work with left-hand sides

$$
\int_{\partial \mathscr{A}}\left(\mathbb{B}^{\top} \boldsymbol{a}\right) \cdot \boldsymbol{v}_{\partial \mathscr{A}} \mathrm{d} s, \quad \int_{\partial \mathscr{A}}\left(\mathbb{B}^{\top} \boldsymbol{h}\right) \cdot \boldsymbol{v}_{\partial \mathscr{A}} \mathrm{d} s,
$$

using (5.2).

\subsection{Standard momentum and moment of momentum balance}

In addition to the bulk Cauchy stress $\boldsymbol{T}$, we account for a standard surface stress $\mathbb{T}$, which is a superficial tensor field on $\mathscr{S}$. Let $\mathscr{R}(t)$ be an arbitrary two-phase migrating control volume, with $\mathscr{A}(t)$ the portion of $\mathscr{S}(t)$ in $\mathscr{R}(t)$. Then the integral of the 
traction $\mathbb{T} \boldsymbol{v}_{\partial \mathscr{A}}$ over $\partial \mathscr{A}(t)$ represents the force exerted on $\mathscr{R}(t)$ by the portion of $\mathscr{S}$ exterior to $\mathscr{R}(t)$; the balances of standard momentum and moment of momentum for $\mathscr{R}(t)$ therefore have the form (bearing in mind that we neglect interfacial mass)

$$
\frac{\mathrm{d}}{\mathrm{d} t} \int_{\mathscr{R}(t)} \varrho \boldsymbol{u} \mathrm{d} v=\int_{\partial \mathscr{R}(t)}\left\{\boldsymbol{T} \boldsymbol{m}+\boldsymbol{u} J_{\partial \mathscr{R}}\right\} \mathrm{d} a+\int_{\partial \mathscr{A}(t)} \mathbb{T} \boldsymbol{v}_{\partial \mathscr{A}} \mathrm{d} s
$$

and

$$
\frac{\mathrm{d}}{\mathrm{d} t} \int_{\mathscr{R}(t)}(\boldsymbol{x}-\mathbf{0}) \times \varrho \boldsymbol{u} \mathrm{d} v=\int_{\partial \mathscr{R}(t)}(\boldsymbol{x}-\mathbf{0}) \times\left\{\boldsymbol{T} \boldsymbol{m}+\boldsymbol{u} J_{\partial \mathscr{R}}\right\} \mathrm{d} a+\int_{\partial \mathscr{A}(t)}(\boldsymbol{x}-\mathbf{0}) \times \mathbb{\Gamma} \boldsymbol{v}_{\partial \mathscr{A}} \mathrm{d} s .
$$

Consider the momentum balance (5.3). The localization of this balance hinges on the distillation of $\int_{\partial \mathscr{R}}\left\{\boldsymbol{T} \boldsymbol{m}+\boldsymbol{u} J_{\partial \mathscr{R}}\right\} \mathrm{d} a$. To achieve this distillation, we first note that, by virtue of (4.32),

$$
\int_{\partial \mathscr{R}(t)} \boldsymbol{T} \boldsymbol{m} \mathrm{d} a=\int_{\partial \mathscr{R}^{+}(t)} \boldsymbol{T} \boldsymbol{m} \mathrm{d} a+\int_{\partial \mathscr{R}^{-}(t)} \boldsymbol{T} \boldsymbol{m} \mathrm{d} a+\int_{\mathscr{A}(t)} \llbracket \boldsymbol{T} \rrbracket n \mathrm{~d} a ;
$$

Next, to distill the term $\int_{\partial \mathscr{R}} \boldsymbol{u} J_{\partial \mathscr{R}} \mathrm{d} a$, we note that, by (3.3), (4.13a), (4.32) and (4.33),

$$
J_{\partial \mathscr{R}^{+}}=\left\{\begin{array}{ll}
J_{\partial \mathscr{R}} & \text { on } \partial \mathscr{R}_{b l k}^{+}, \\
-J & \text { on } \mathscr{A},
\end{array} \quad J_{\partial \mathscr{T}^{-}}= \begin{cases}J_{\partial \mathscr{R}} & \text { on } \partial \mathscr{R}_{b l k}^{-}, \\
J & \text { on } \mathscr{A},\end{cases}\right.
$$

so that, bearing in mind $(4.13 b)$ and (4.15),

$$
\begin{aligned}
\int_{\partial \mathscr{R}(t)} \boldsymbol{u} J_{\partial \mathscr{R}} \mathrm{d} a & =\int_{\partial \mathscr{R}^{+}(t)} \boldsymbol{u} J_{\partial \mathscr{R}} \mathrm{d} a+\int_{\partial \mathscr{R}^{-}(t)} \boldsymbol{u} J_{\partial \mathscr{R}} \mathrm{d} a+\int_{\mathscr{A}(t)} \llbracket \boldsymbol{u} \rrbracket J \mathrm{~d} a \\
& =\int_{\partial \mathscr{R}^{+}(t)} \boldsymbol{u} J_{\partial \mathscr{R}} \mathrm{d} a+\int_{\partial \mathscr{R}^{-}(t)} \boldsymbol{u} J_{\partial \mathscr{R}} \mathrm{d} a-\int_{\mathscr{A}(t)} J^{2} \llbracket v \rrbracket \boldsymbol{n} \mathrm{d} a
\end{aligned}
$$

Trivially, $(\mathrm{d} / \mathrm{d} t) \int_{\mathscr{R}} \varrho \boldsymbol{u} \mathrm{d} v=(\mathrm{d} / \mathrm{d} t) \int_{\mathscr{R}^{+}} \varrho \boldsymbol{u} \mathrm{d} v+(\mathrm{d} / \mathrm{d} t) \int_{\mathscr{R}^{-}} \varrho \boldsymbol{u} \mathrm{d} v$; thus, appealing to (5.5) and (5.7), we may rewrite (5.3) in the form

$$
\begin{aligned}
& \underbrace{\frac{\mathrm{d}}{\mathrm{d} t} \int_{\mathscr{R}^{+}(t)} \varrho \boldsymbol{u} \mathrm{d} v-\int_{\partial \mathscr{R}^{+}(t)}\left\{\boldsymbol{T} \boldsymbol{m}+\boldsymbol{u} J_{\partial \mathscr{R}}\right\} \mathrm{d} a}_{=\mathbf{0} \text { by }(3.5 a)}+\underbrace{\frac{\mathrm{d}}{\mathrm{d} t} \int_{\mathscr{R}^{-}(t)} \varrho \boldsymbol{u} \mathrm{d} v-\int_{\partial \mathscr{R}^{-}(t)}\left\{\boldsymbol{T} \boldsymbol{m}+\boldsymbol{u} J_{\partial \mathscr{R}}\right\} \mathrm{d} a}_{=\mathbf{0} \text { by }(3.5 a)} \\
& =\int_{\mathscr{A}(t)}\left\{\llbracket \boldsymbol{T} \rrbracket \boldsymbol{n}-J^{2} \llbracket v \rrbracket \boldsymbol{n}\right\} \mathrm{d} a+\int_{\partial \mathscr{A}(t)} \mathbb{T} \boldsymbol{v}_{\partial \mathscr{A}} \mathrm{d} s ;
\end{aligned}
$$

therefore, we have the first of the interfacial balances

$$
\begin{gathered}
\int_{\mathscr{A}(t)}\left\{\llbracket \boldsymbol{T} \rrbracket \boldsymbol{n}-J^{2} \llbracket v \rrbracket \boldsymbol{n}\right\} \mathrm{d} a+\int_{\partial \mathscr{A}(t)} \mathbb{T} \boldsymbol{v}_{\partial \mathscr{A}} \mathrm{d} s=\mathbf{0}, \\
\int_{\mathscr{A}(t)}(\boldsymbol{x}-\mathbf{0}) \times\left\{\llbracket \boldsymbol{T} \rrbracket \boldsymbol{n}-J^{2} \llbracket v \rrbracket \boldsymbol{n}\right\} \mathrm{d} a+\int_{\partial \mathscr{A}(t)}(\boldsymbol{x}-\mathbf{0}) \times \mathbb{T} \boldsymbol{v}_{\partial \mathscr{A}} \mathrm{d} s=\mathbf{0},
\end{gathered}
$$

and the second follows similarly. Given any migrating subsurface $\mathscr{A}(t)$ of $\mathscr{S}(t)$, there is at least one two-phase migrating control volume $\mathscr{R}(t)$ such that $\mathscr{A}(t)=\mathscr{R}(t) \cap \mathscr{S}(t)$; indeed, choose $\delta>0$ sufficiently small and let $\mathscr{R}(t)=\mathscr{R}_{\delta}(t)$ be the $\delta$-pillbox about $\mathscr{A}(t)$ :

$$
\mathscr{R}_{\delta}(t) \stackrel{\text { def }}{=}\{\boldsymbol{x}|\boldsymbol{x}=\boldsymbol{y} \pm \lambda \boldsymbol{n}(\boldsymbol{y}, t), \boldsymbol{y} \in \mathscr{A}(t),| \lambda \mid \leqslant \delta\} .
$$

Thus, the balances (5.9) must be satisfied for all migrating subsurface $\mathscr{A}(t)$ of $\mathscr{S}(t)$. 
Localizing (5.9), we are led to the relations

$$
\operatorname{div}_{\mathscr{S}} \mathbb{T}+\llbracket \boldsymbol{T} \rrbracket \boldsymbol{n}=J^{2} \llbracket v \rrbracket n, \quad \mathbb{T}=\mathbb{T}^{\top},
$$

and the second of these implies that $\mathbb{T}$ is fully tangential.

Equation (5.11a) follows on applying the surface divergence theorem in the form (5.1a) to (5.9a). To derive the second, let $\boldsymbol{a}$ an arbitrary constant vector, let $(\boldsymbol{a} \times)$ denote the tensor defined by $(\boldsymbol{a} \times) \boldsymbol{b}=\boldsymbol{a} \times \boldsymbol{b}$ for every vector $\boldsymbol{b}$, and note that $\operatorname{grad}_{\mathscr{G}}(\boldsymbol{a} \times \boldsymbol{x})=$ $(\boldsymbol{a} \times)(\operatorname{grad} \boldsymbol{x}) \mathbb{P}=(\boldsymbol{a} \times) \mathbb{P}$. Then, by $(5.1 b)$ and $(5.11 a)$,

$$
\begin{aligned}
\boldsymbol{a} \cdot \int_{\partial \mathscr{A}(t)} \boldsymbol{x} \times \mathbb{T} \boldsymbol{v}_{\partial \mathscr{A}} \mathrm{d} s & =\int_{\partial \mathscr{A}(t)}(\boldsymbol{a} \times \boldsymbol{x}) \cdot \mathbb{T} \boldsymbol{v}_{\partial \mathscr{A}} \mathrm{d} s \\
& =\int_{\mathscr{A}(t)}\left\{\operatorname{div}_{\mathscr{S}} \mathbb{T} \cdot(\boldsymbol{a} \times \boldsymbol{x})+\mathbb{T}: \operatorname{grad}_{\mathscr{A}}(\boldsymbol{a} \times \boldsymbol{x})\right\} \mathrm{d} a \\
& =\int_{\mathscr{A}(t)}\left\{\boldsymbol{a} \cdot\left(\boldsymbol{x} \times \operatorname{div}_{\mathscr{S}} \mathbb{T}\right)+\mathbb{T}:((\boldsymbol{a} \times) \mathbb{P})\right\} \mathrm{d} a \\
& =-\boldsymbol{a} \cdot \int_{\mathscr{A}(t)} \boldsymbol{x} \times\left\{\llbracket \boldsymbol{T} \rrbracket \boldsymbol{n}-J^{2} \llbracket v \rrbracket \boldsymbol{n}\right\} \mathrm{d} a+\int_{\mathscr{A}(t)} \mathbb{T}:\{(\boldsymbol{a} \times) \mathbb{P}\} \mathrm{d} a .
\end{aligned}
$$

Further, since $\mathbb{T}$ is a superficial tensor, $\mathbb{T}:\{(\boldsymbol{a} \times) \mathbb{P}\}=(\mathbb{T} \mathbb{P}):(\boldsymbol{a} \times)=\mathbb{T}:(\boldsymbol{a} \times)$ and, by $(5.9 b)$ and (5.12), $\int_{\mathscr{A}} \mathbb{T}:(\boldsymbol{a} \times) \mathrm{d} a=0$. Therefore, since $\mathscr{A}$ is arbitrary, $\mathbb{T}:(\boldsymbol{a} \times)=0$ for every $\boldsymbol{a}$. However, any skew tensor may be written in the form $\boldsymbol{a} \times$; thus $\mathbb{T}$ is symmetric and $(5.11 b)$ holds.

Since $\mathbb{T}$ is fully tangential, (4.9) implies that $\boldsymbol{n} \cdot \operatorname{div}_{\mathscr{S}} \mathbb{T}=\mathbb{T}: \mathbb{K}$ thereby rendering the normal part of the standard momentum balance (5.11a) of the form

$$
\mathbb{T}: \mathbb{K}+\boldsymbol{n} \cdot \llbracket \boldsymbol{T} \rrbracket \boldsymbol{n}=J^{2} \llbracket v \rrbracket .
$$

\subsection{Configurational momentum balance}

Next, in addition to the stress tensor $\boldsymbol{C}$ and the internal body force $\boldsymbol{f}$ in bulk, we allow for a configurational surface stress $\mathbb{C}$, which is a superficial tensor field on $\mathscr{S}$, and an internal configurational surface force $\boldsymbol{f}^{x}$, with $\boldsymbol{f}^{x}$ a vector field on $\mathscr{S}$.

We posit a balance law for configurational forces asserting that, for any two-phase migrating control volume,

$$
\frac{\mathrm{d}}{\mathrm{d} t} \int_{\mathscr{R}(t)} \varrho \boldsymbol{p} \mathrm{d} v=\int_{\partial \mathscr{R}(t)}\left\{\boldsymbol{C} \boldsymbol{m}+\boldsymbol{p} J_{\partial \mathscr{A}}\right\} \mathrm{d} a+\int_{\mathscr{R}(t)} \boldsymbol{f} \mathrm{d} v+\int_{\partial \mathscr{A}(t)} \mathbb{C}_{\partial \mathscr{\partial}} \mathrm{d} s+\int_{\mathscr{A}(t)} \boldsymbol{f}^{x} \mathrm{~d} a
$$

(cf. §5.1). Then, arguing as we did in going from (5.5) to (5.9a) and making use of the momentum relation (3.17), we obtain

$$
\int_{\mathscr{A}(t)}\left\{\llbracket \boldsymbol{C} \rrbracket \boldsymbol{n}+J^{2} \llbracket v \rrbracket \boldsymbol{n}\right\} \mathrm{d} a+\int_{\partial \mathscr{A}(t)} \mathbb{C} \boldsymbol{v}_{\partial \mathscr{A}} \mathrm{d} s+\int_{\mathscr{A}(t)} \boldsymbol{f}^{x} \mathrm{~d} a=\mathbf{0},
$$

for any migrating subsurface $\mathscr{A}(t) \subset \mathscr{S}(t)$. Thus

$$
\operatorname{div}_{\mathscr{S}} \mathbb{C}+\boldsymbol{f}^{x}+\llbracket \boldsymbol{C} \rrbracket \boldsymbol{n}+J^{2} \llbracket v \rrbracket \boldsymbol{n}=\mathbf{0} .
$$

We let

$$
f=\boldsymbol{f}^{x} \cdot \boldsymbol{n}
$$


denote the normal internal configurational force on the interface. The balance (5.16) may then be decomposed into a normal configurational momentum balance

$$
\boldsymbol{n} \cdot \operatorname{div}_{\mathscr{S}} \mathbb{C}+f+\boldsymbol{n} \cdot \llbracket \boldsymbol{C} \rrbracket \boldsymbol{n}+J^{2} \llbracket v \rrbracket=\mathbf{0}
$$

and a tangential balance that is irrelevant to what follows (cf. Remark (ii) after $(6.20))$.

Finally, in view of (4.4), the configurational stress admits the decomposition

$$
\mathbb{C}=\mathbb{C}_{\tan }+\boldsymbol{n} \otimes \boldsymbol{c},
$$

in which $\mathbb{C}_{\text {tan }}$, which is fully tangential, characterizes configurational stress within the interface, while the tangential vector $\boldsymbol{c}$ characterizes configurational surface shear; i.e. for any tangent vector $\boldsymbol{v}$,

$$
\mathbb{C} \boldsymbol{v}=\mathbb{C}_{\tan } \boldsymbol{v}+(\boldsymbol{c} \cdot \boldsymbol{v}) \boldsymbol{n} .
$$

Next, since

$$
\boldsymbol{n} \cdot \operatorname{div}_{\mathscr{f}}(\boldsymbol{n} \otimes \boldsymbol{c})=\operatorname{div}_{\mathscr{c}} \boldsymbol{c}+\underbrace{\boldsymbol{n} \cdot\left(\left(\operatorname{grad}_{\mathscr{f}} \boldsymbol{n}\right) \boldsymbol{c}\right)}_{=-\mathbb{K} n \cdot \boldsymbol{c}=0}=\operatorname{div}_{\mathscr{f}} \boldsymbol{c},
$$

we may use (4.9) and (5.19) to write the normal configurational momentum balance (5.18) in the form

$$
\operatorname{div}_{s} \boldsymbol{c}+\mathbb{C}_{\mathrm{tan}}: \mathbb{K}+f+\boldsymbol{n} \cdot \llbracket \boldsymbol{C} \rrbracket \boldsymbol{n}+\boldsymbol{J}^{2} \llbracket v \rrbracket=\mathbf{0} .
$$

\subsection{Power expended by standard and configurational forces}

\section{Total power expenditure}

Let $\mathscr{A}(t)$ be a migrating subsurface of $\mathscr{S}(t)$. Then guided by the discussion of $\S 3$, we take the observed velocity $\boldsymbol{v}_{\partial \alpha}$ of $\partial \mathscr{A}$ as the power conjugate velocity for the standard traction $\mathbb{T} \boldsymbol{v}_{\partial \mathscr{A}}$ on $\partial \mathscr{A}$. For the configurational traction $\mathbb{C} \boldsymbol{v}_{\partial \mathscr{A}}$, a velocity for the migration of $\mathscr{A}$ would be appropriate, but there are two values of the velocity $\boldsymbol{u}$ at the surface. For that reason it seems reasonable to use the average migrational velocity

$$
\boldsymbol{v}_{\partial \varkappa \mathcal{L}}-\langle\boldsymbol{u}\rangle
$$

of $\partial \mathscr{A}$ as the appropriate power conjugate velocity for $\mathbb{C} \boldsymbol{v}_{\partial \mathscr{A}}$.

Let $\mathscr{R}(t)$ denote an arbitrary two-phase migrating control volume, with $\mathscr{A}(t)$ the portion of $\mathscr{S}(t)$ in $\mathscr{R}(t)$. The integral that represents $W(\mathscr{R}(t))$ in (3.10) here gives the power expended on that portion of $\partial \mathscr{R}(t)$ that lies in the bulk phases; for that reason we write the power expended on $\mathscr{R}(t)$ in the form

$$
\begin{aligned}
& W_{\text {tot }}(\mathscr{R}(t))=\int_{\partial \mathscr{A}(t)}\left\{\mathbb{T} \boldsymbol{v}_{\partial \mathscr{c}} \cdot \boldsymbol{v}_{\partial \mathscr{\alpha}}+\mathbb{C} \boldsymbol{v}_{\partial \mathscr{\alpha}} \cdot\left(\boldsymbol{v}_{\partial \mathscr{\alpha}}-\langle\langle\boldsymbol{u}\rangle)\right\} \mathrm{d} s\right. \\
& +\underbrace{\int_{\partial \mathscr{R}(t)}\left\{\left(\boldsymbol{T} \boldsymbol{m}+\boldsymbol{u} J_{\partial \Re}\right) \cdot \boldsymbol{v}_{\partial \Re}+\left(\boldsymbol{C} \boldsymbol{m}-\boldsymbol{u} J_{\partial 囚}\right) \cdot\left(\boldsymbol{v}_{\partial \Re}-\boldsymbol{u}\right)\right\} \mathrm{d} a}_{W(\mathscr{R}(t))} .
\end{aligned}
$$

Here, we use the momentum relation (3.17). Because the configurational surface force $f^{x}$ is internal, it does not enter the power expenditure (5.21).

Interfacial power expenditure. Pre-Eshelby relation

We continue to use the notation and terminology of $\S 4.8$.

The term $W(\mathscr{R}(t))$ leads to interfacial contributions. To determine those contributions we first use (4.32), (4.33) and (5.6) to obtain the distillations 


$$
\begin{aligned}
\int_{\partial \mathscr{R}(t)}\left\{\boldsymbol{T m}+\boldsymbol{u} J_{\partial \mathscr{R}}\right\} \cdot \boldsymbol{v}_{\partial \mathscr{R}} \mathrm{d} a=\int_{\partial \mathscr{R}^{+}(t)}\left\{\boldsymbol{T} \boldsymbol{m}+\boldsymbol{u} J_{\partial \mathscr{R}^{+}}\right\} \cdot \boldsymbol{v}_{\partial \mathscr{R}^{+}} \mathrm{d} a \\
+\int_{\partial \mathscr{R}^{-}(t)}\left\{\boldsymbol{T} \boldsymbol{m}+\boldsymbol{u} J_{\partial \mathscr{R}^{-}}\right\} \cdot \boldsymbol{v}_{\partial \mathscr{R}^{-}} \mathrm{d} a+\int_{\mathscr{A}(t)} \overbrace{\left\{\llbracket \boldsymbol{T} \rrbracket \boldsymbol{n}-J^{2} \llbracket v \rrbracket \boldsymbol{n}\right\}}^{=-\operatorname{div} \mathscr{T} \mathbb{T} \text { by }(5.11 a)} \cdot \boldsymbol{v} \mathrm{d} a
\end{aligned}
$$

and

$$
\begin{gathered}
\int_{\partial \mathscr{R}(t)}\left\{\boldsymbol{C m}-\boldsymbol{u} J_{\partial \mathscr{K}}\right\} \cdot\left(\boldsymbol{v}_{\partial \mathscr{R}}-\boldsymbol{u}\right) \mathrm{d} a=\int_{\partial \mathscr{R}^{+}(t)}\left\{\boldsymbol{C m}-\boldsymbol{u} J_{\partial \mathscr{R}^{+}}\right\} \cdot\left(\boldsymbol{v}_{\partial \mathscr{R}^{+}}-\boldsymbol{u}\right) \mathrm{d} a \\
+\int_{\partial \mathscr{R}^{-}(t)}\left\{\boldsymbol{C m}-\boldsymbol{u} J_{\partial \mathscr{R}^{-}}\right\} \cdot\left(\boldsymbol{v}_{\partial \mathscr{R}^{-}}-\boldsymbol{u}\right) \mathrm{d} a+\int_{\mathscr{A}(t)} \llbracket(\boldsymbol{C} \boldsymbol{n}-\boldsymbol{u} J) \cdot(\boldsymbol{v}-\boldsymbol{u}) \rrbracket \mathrm{d} a .
\end{gathered}
$$

These distillations yield the decomposition

$$
W(\mathscr{R}(t))=W\left(\mathscr{R}^{+}(t)\right)+W\left(\mathscr{R}^{-}(t)\right)-\int_{\mathscr{A}(t)}\left\{\boldsymbol{v} \cdot \operatorname{div}_{\mathscr{S}} \mathrm{T}-\llbracket(\boldsymbol{C} n-\boldsymbol{u} J) \cdot(\boldsymbol{v}-\boldsymbol{u}) \rrbracket\right\} \mathrm{d} a ;
$$

(5.21) therefore becomes

$$
W_{\text {tot }}(\mathscr{R}(t))=W\left(\mathscr{R}^{+}(t)\right)+W\left(\mathscr{R}^{-}(t)\right)+w(\mathscr{A}(t)),
$$

with

$$
\begin{aligned}
w(\mathscr{A}(t))=\underbrace{\int_{\partial \mathscr{A}(t)}\left\{\boldsymbol{T} \boldsymbol{v}_{\partial \mathscr{A}} \cdot \boldsymbol{v}_{\partial \mathscr{A}}+\boldsymbol{C}_{\partial \mathscr{A}} \cdot\left(\boldsymbol{v}_{\partial \mathscr{A}}-\langle\langle\boldsymbol{u}\rangle)\right\} \mathrm{d} s\right.}_{w^{*}(\mathscr{A}(t))} \\
\quad-\int_{\mathscr{A}(t)}\left\{\boldsymbol{v} \cdot \operatorname{div}_{\mathscr{S}} \mathbb{T}-\llbracket(\boldsymbol{C} \boldsymbol{n}-\boldsymbol{u} J) \cdot(\boldsymbol{v}-\boldsymbol{u}) \rrbracket\right\} \mathrm{d} a
\end{aligned}
$$

the interfacial power expenditure for the migrating subsurface $\mathscr{A}(t)$.

We assume that the intrinsicality hypothesis applies also for two-phase migrating control volumes. In view of the bulk Eshelby relation (3.16) and the momentum relation (3.17), it follows that $W\left(\mathscr{R}^{+}\right)$and $W\left(\mathscr{R}^{-}\right)$are independent of the velocity fields $\boldsymbol{v}_{\partial \mathscr{R}^{+}}$and $\boldsymbol{v}_{\partial \mathscr{R}^{-}}$for $\partial \mathscr{R}^{+}$and $\partial \mathscr{R}^{-}$. Thus, by (5.22), intrinsicality is equivalent to the stipulation that $w(\mathscr{A}(t))$ be independent of the choice of observed velocity field $\boldsymbol{v}_{\partial \mathscr{A}}$ chosen to characterize the migration of $\mathscr{A}(t)$ (cf. the intrinsicality hypothesis in $\S 3.1$ ). Equivalently, by $(5.23)$, we require that $w^{*}(\mathscr{A})$ be invariant under all transformations of the form (4.24); thus, necessary and sufficient that the above stipulation be satisfied is that the terms $w^{*}(\mathscr{A})$ have this invariance, or, equivalently, that

$$
\int_{\partial \mathscr{A}} \boldsymbol{t} \cdot\left\{\boldsymbol{T} \boldsymbol{v}_{\partial \mathscr{A}}+\mathbb{C} \boldsymbol{v}_{\partial \mathscr{A}}\right\} \mathrm{d} s=\mathbf{0}
$$

for all subsurfaces $\mathscr{A}$ of $\mathscr{S}$ and all fields $t$ tangential to $\partial \mathscr{A}$. Recalling that $\mathbb{T}$ is fully tangential and noting that only the fully tangential part $\mathbb{C}_{\text {tan }}$ of $\mathbb{C}$ is affected by (5.24), we see that this requirement is equivalent to the requirement that the superficial field $\mathbb{B}=\mathbb{T}+\mathbb{C}$ satisfy

$$
\boldsymbol{t} \cdot \mathbb{B} v=0
$$

at any point $\boldsymbol{x}$ of $\mathscr{S}$ whenever $\boldsymbol{t}$ and $\boldsymbol{v}$ are orthogonal and tangent to $\mathscr{S}$ at $\boldsymbol{x}$. Arguing as in the derivation of (3.19), we find that there must exist a superficial scalar field $\varphi$ 
such that

$$
\mathbb{C}_{\text {tan }}=\varphi \mathbb{P}-\mathbb{T},
$$

which represents a pre-Eshelby relation for the interface that is analogous to the bulk relation (3.19). In the absence of configurational surface stress $\mathbb{T}=\varphi \mathbb{P}$, so that $\varphi$ plays the role of surface tension; but this is not the case when both configurational and standard surface stresses are present, for then, by (5.25), $\varphi$ plays the role of a 'surface tension' for the combined stress $\mathbb{T}+\mathbb{C}_{\text {tan }}$.

Using (4.20), (4.20a), (5.19) and (5.25), we find that

$$
\begin{aligned}
& \mathbb{T} \boldsymbol{v}_{\partial \alpha} \cdot \boldsymbol{v}_{\partial \alpha}+\mathbb{C} \boldsymbol{v}_{\partial \alpha} \cdot\left(\boldsymbol{v}_{\partial \alpha}-\langle\langle\boldsymbol{u}\rangle)=\mathbb{T} \boldsymbol{v}_{\partial \alpha} \cdot \boldsymbol{v}+\mathbb{C} \boldsymbol{v}_{\partial \alpha} \cdot\left(\boldsymbol{v}-\langle\langle\boldsymbol{u}\rangle)+(\mathbb{T}+\mathbb{C}) \boldsymbol{v}_{\partial \alpha} \cdot\left(\boldsymbol{v}_{\partial \alpha}-\boldsymbol{v}\right)\right.\right. \\
& =\mathbb{T} \boldsymbol{v}_{\partial \alpha,} \cdot \boldsymbol{v}+\mathbb{C} \boldsymbol{v}_{\partial \alpha,} \cdot(\boldsymbol{v}-\langle u \boldsymbol{u}\rangle)+\varphi\left(\boldsymbol{v}_{\partial \alpha}-\boldsymbol{v}\right) \cdot \boldsymbol{v}_{\partial \alpha}+\left(\boldsymbol{c} \cdot \boldsymbol{v}_{\partial \alpha}\right) \underbrace{\left(\boldsymbol{v}_{\partial \alpha}-\boldsymbol{v}\right) \cdot \boldsymbol{n}}_{=\mathbf{0}},
\end{aligned}
$$

and thus, by $(5.1 b)$, that

$$
\begin{aligned}
& w^{*}(\mathscr{A}(t))-\int_{\partial \mathscr{A}(t)} \varphi\left(\boldsymbol{v}_{\partial \mathscr{A}}-\boldsymbol{v}\right) \cdot \boldsymbol{v}_{\partial \mathscr{\ell}} \mathrm{d} s=\int_{\partial \mathscr{A}(t)}\left\{\mathbb{T} \boldsymbol{v}_{\partial \mathscr{\ell}} \cdot \boldsymbol{v}+\mathbb{C}_{\partial \mathscr{A}} \cdot(\boldsymbol{v}-\langle\langle\boldsymbol{u}\rangle)\} \mathrm{d} s\right. \\
= & \int_{\mathscr{A}(t)}\left\{\boldsymbol{v} \cdot \operatorname{div}_{\mathscr{S}} \mathbb{T}+(\boldsymbol{v}-\langle\boldsymbol{u}\rangle) \cdot \operatorname{div}_{\mathscr{S}} \mathbb{C}+\mathbb{T}: \operatorname{grad}_{\mathscr{S}} \boldsymbol{v}+\mathbb{C}: \operatorname{grad}_{\mathscr{f}}(\boldsymbol{v}-\langle\langle\boldsymbol{u}\rangle)\} \mathrm{d} a .\right.
\end{aligned}
$$

Further, noting that, by (4.10), (4.13a), and (4.15),

$$
\begin{aligned}
\llbracket(C n-u J) \cdot(v-u) \rrbracket & =\llbracket(C n-u J) \rrbracket \cdot(v-\langle u\rangle\rangle)+\langle(C n-u J)\rangle \cdot \llbracket v-u \rrbracket \\
& =\llbracket C n-u J \rrbracket \cdot(v-\langle u\rangle\rangle)-\langle\langle C n-u J\rangle\rangle \cdot \llbracket u \rrbracket \\
& =\llbracket C n-u J \rrbracket \cdot(v-\langle u\rangle)+J \llbracket v \rrbracket(n \cdot\langle C\rangle\rangle n-J\langle u u\rangle \cdot n),
\end{aligned}
$$

and that, by (5.19), (5.25) and (4.16)-(4.18),

$$
\begin{aligned}
\mathbb{T}: \operatorname{grad}_{\mathscr{f}} \boldsymbol{v} & +\mathbb{C}: \operatorname{grad}_{\mathscr{f}}(\boldsymbol{v}-\langle\langle\boldsymbol{u}\rangle) \\
& =\underbrace{\left(\mathbb{T}+\mathbb{C}_{\mathrm{tan}}\right): \operatorname{grad}_{\mathscr{P}} \boldsymbol{v}}_{=\varphi \operatorname{div}_{\mathscr{S}} \boldsymbol{v}}+(\boldsymbol{n} \otimes \boldsymbol{c}): \operatorname{grad}_{\mathscr{f}}(\boldsymbol{v}-\langle\boldsymbol{u}\rangle\rangle)-\underbrace{\left.\mathbb{C}_{\tan }: \operatorname{grad}_{\mathscr{P}}\langle\boldsymbol{u}\rangle\right\rangle}_{=\mathbb{C}_{\text {tan }}: \mathbb{D}},
\end{aligned}
$$

we find, on substituting (5.26) into (5.23) and appealing to (4.13) and the force balances (5.11) and (5.16), that

$$
\begin{gathered}
w(\mathscr{A}(t))=\int_{\partial \mathscr{A}(t)} \varphi\left(\boldsymbol{v}_{\partial \mathscr{A}}-\boldsymbol{v}\right) \cdot \boldsymbol{v}_{\partial \mathscr{A}} \mathrm{d} s+\int_{\mathscr{A}(t)}\left\{\varphi \operatorname{div}_{\mathscr{S}} \boldsymbol{v}-\mathbb{C}_{\tan }: \mathbb{D}-\boldsymbol{f}^{\mathrm{x}} \cdot(\boldsymbol{v}-\langle\boldsymbol{u}\rangle)\right\} \mathrm{d} a \\
\left.\quad+\int_{\mathscr{A}(t)}\left\{(\boldsymbol{n} \otimes \boldsymbol{c}): \operatorname{grad}_{\mathscr{A}}(\boldsymbol{v}-\langle\langle\boldsymbol{u}\rangle\rangle)+J \llbracket v \rrbracket(\boldsymbol{n} \cdot\langle\boldsymbol{C}\rangle\rangle \boldsymbol{n}-J\langle\boldsymbol{u}\rangle \cdot \boldsymbol{n}\right)\right\} \mathrm{d} a . \quad(5.27)
\end{gathered}
$$

We henceforth require that $\boldsymbol{v}$ be the migrationally normal velocity field for $\mathscr{S}$ as defined in (4.27), so that, recalling (4.23), (4.28) and (4.8):

$$
\left(\boldsymbol{v}_{\partial \alpha}-\boldsymbol{v}\right) \cdot \boldsymbol{v}_{\partial \alpha}=V_{\partial \alpha,}^{m i g}, \quad \boldsymbol{v}-\left\langle\langle\boldsymbol{u}\rangle=V^{m i g} \boldsymbol{n}, \quad \operatorname{div}_{\mathscr{S}} \boldsymbol{v}=\operatorname{div}_{\mathscr{S}} \boldsymbol{u}_{t a n}-K V .\right.
$$

Hence, by (4.13) and (4.28b),

$$
\left.(\boldsymbol{n} \otimes \boldsymbol{c}): \operatorname{grad}_{\mathscr{C}}(\boldsymbol{v}-\langle\boldsymbol{u}\rangle\rangle\right)=\boldsymbol{c} \cdot \operatorname{grad}_{\mathscr{S}} V^{m i g}, \quad \boldsymbol{f}^{x} \cdot(\boldsymbol{v}-\langle\boldsymbol{u}\rangle)=\langle\langle v\rangle f J,
$$


and (5.27) takes the intrinsic form

$w(\mathscr{A}(t))=\int_{\partial \mathscr{A}(t)} \varphi V_{\partial \mathscr{\partial}}^{m i g} \mathrm{~d} s-\int_{\mathscr{A}(t)}\left\{\varphi\left(K V-\operatorname{div}_{\mathscr{S}} \boldsymbol{u}_{t a n}\right)+\mathbb{C}_{t a n}: \mathbb{D}-\boldsymbol{c} \cdot \operatorname{grad}_{\mathscr{L}} V^{m i g}+F J\right\} \mathrm{d} a$,

with

$$
F=\langle\langle v\rangle f-\llbracket v \rrbracket \boldsymbol{n} \cdot(\langle\langle\boldsymbol{C}\rangle\rangle \boldsymbol{n}-J\langle\boldsymbol{u}\rangle\rangle \cdot \boldsymbol{n}) .
$$

Equation (5.30) represents the final form of the expended power.

\section{Interfacial forms of the first two laws}

6.1. Global statements of the first two laws

In this subsection we introduce global statements of the first two laws appropriate to a migrating control volume that contains a portion of the interface. Here, we find it most useful to use appropriate generalizations of the bulk laws in configurational form as given in (3.10) and (3.11).

We assume that the temperature $\vartheta$ is continuous across the interface, namely,

$$
\llbracket \vartheta \rrbracket=0 .
$$

The restriction of $\vartheta$ to $\mathscr{S}$ then represents the temperature of the interface. We endow the interface with internal energy and entropy (densities) $\varepsilon^{x}$ and $\eta^{x}$, but neglect interfacial heat flux. We write $\psi^{x}$ for the interfacial free energy (density)

$$
\psi^{x}=\varepsilon^{x}-\vartheta \eta^{x} .
$$

Let $\mathscr{A}(t)$ be an arbitrary migrating subsurface of $\mathscr{S}(t)$. Consider the first two laws in bulk as expressed by (3.10) and (3.11), and, in particular, the terms

$$
\int_{\partial \mathscr{R}(t)} \vartheta \eta J_{\partial \mathscr{}} \mathrm{d} a, \quad \int_{\partial \mathscr{R}(t)} \eta J_{\partial \Re} \mathrm{d} a,
$$

which represent flows of heat and entropy induced by the migration of $\partial \mathscr{R}$. Guided by (6.3), we view

$$
\int_{\partial \mathscr{A}(t)} \vartheta \eta^{x} V_{\partial \mathscr{A}}^{m i g} \mathrm{~d} s, \quad \int_{\partial \mathscr{A}(t)} \eta^{x} V_{\partial \mathscr{c}}^{m i g} \mathrm{~d} s,
$$

respectively, as flows of heat and entropy induced by the migration of $\partial \mathscr{A}$. We use $V_{\partial \mathscr{A}}^{\text {mig }}$ (cf. (4.23) - the normal migrational velocity of $\partial \mathscr{A}$ - because we do not allow for mass flow within the interface.

Then, given any two-phase migrating control volume $\mathscr{R}(t)$, with $\mathscr{A}(t)$ the portion of $\mathscr{S}(t)$ in $\mathscr{R}(t)$, the first two laws for $\mathscr{R}(t)$ have the form

$$
\begin{aligned}
\frac{\mathrm{d}}{\mathrm{d} t} \int_{\mathscr{R}(t)} \varrho\left\{\varepsilon+\frac{1}{2}|\boldsymbol{u}|^{2}\right\} \mathrm{d} v+\frac{\mathrm{d}}{\mathrm{d} t} \int_{\mathscr{A}(t)} \varepsilon^{x} \mathrm{~d} a \\
=W_{\text {tot }}(\mathscr{R}(t))+\int_{\partial \mathscr{R}(t)}\left\{\vartheta \eta J_{\partial \mathscr{A}}-\boldsymbol{q} \cdot \boldsymbol{m}\right\} \mathrm{d} a+\int_{\partial \mathscr{A}(t)} \vartheta \eta^{x} V_{\partial \mathscr{A}}^{m i g} \mathrm{~d} s,
\end{aligned}
$$

with $W_{\text {tot }}(\mathscr{R}(t))$ defined in (5.22), and

$$
\frac{\mathrm{d}}{\mathrm{d} t} \int_{\mathscr{R}(t)} \varrho \eta \mathrm{d} v+\frac{\mathrm{d}}{\mathrm{d} t} \int_{\mathscr{A}(t)} \eta^{x} \mathrm{~d} a \geqslant \int_{\partial \mathscr{R}(t)} \frac{\vartheta \eta J_{\partial \mathscr{X}}-\boldsymbol{q} \cdot \boldsymbol{m}}{\vartheta} \mathrm{d} a+\int_{\partial \mathscr{A}(t)} \eta^{x} V_{\partial \mathscr{A}}^{m i g} \mathrm{~d} s .
$$


6.2. Localizing the first two laws to the interface. Interfacial Eshelby relation

Step 1. Localization to integral laws involving only the interface

We consider first the energy balance and begin with the distillation

$$
\int_{\partial \mathscr{R}} \boldsymbol{q} \cdot \boldsymbol{m} \mathrm{d} a=\int_{\partial \mathscr{R}^{+}} \boldsymbol{q} \cdot \boldsymbol{m} \mathrm{d} a+\int_{\partial \mathscr{R}^{-}} \boldsymbol{q} \cdot \boldsymbol{m} \mathrm{d} a+\int_{\mathscr{A}} \llbracket \boldsymbol{q} \rrbracket \cdot \boldsymbol{n} \mathrm{d} a .
$$

Next, we use (5.6) to distil the term $\int_{\partial \mathscr{R}} \vartheta \eta J_{\partial \Re} \mathrm{d} a$, giving

$$
\int_{\partial \mathscr{R}} \vartheta \eta J_{\partial \mathscr{R}} \mathrm{d} a=\int_{\partial \mathscr{R}^{+}} \vartheta \eta J_{\partial \mathscr{R}^{+}} \mathrm{d} a+\int_{\partial \mathscr{R}^{-}} \vartheta \eta J_{\partial \mathscr{R}^{-}} \mathrm{d} a+\int_{\mathscr{A}} \vartheta \llbracket \eta \rrbracket J \mathrm{~d} a .
$$

Decomposing the integral $(\mathrm{d} / \mathrm{d} t) \int_{\mathscr{R}} \varrho\left\{\varepsilon+|\boldsymbol{u}|^{2} / 2\right\} \mathrm{d} v$ over $\mathscr{R}(t)$ into contributions over $\mathscr{R}^{+}(t)$ and $\mathscr{R}^{-}(t)$ and appealing to (5.22) and (6.6)-(6.7), we may then rewrite (6.4) in the form

$$
\begin{aligned}
& \underbrace{\frac{\mathrm{d}}{\mathrm{d} t} \int_{\mathscr{R}^{+}(t)} \varrho\left\{\varepsilon+\frac{1}{2}|\boldsymbol{u}|^{2}\right\} \mathrm{d} v-W\left(\mathscr{R}^{+}(t)\right)-Q\left(\mathscr{R}^{+}(t)\right)}_{=\mathbf{0} \text { by }(3.10)} \\
& +\underbrace{\frac{\mathrm{d}}{\mathrm{d} t} \int_{\mathscr{R}^{-}(t)} \varrho\left\{\varepsilon+\frac{1}{2}|\boldsymbol{u}|^{2}\right\} \mathrm{d} v-W\left(\mathscr{R}^{-}(t)\right)-Q\left(\mathscr{R}^{-}(t)\right)}_{=\mathbf{0} \text { by }(3.10)}+\frac{\mathrm{d}}{\mathrm{d} t} \int_{\mathscr{A}(t)} \varepsilon^{x} \mathrm{~d} a \\
& -w(\mathscr{A}(t))-\int_{\mathscr{A}(t)}\{\vartheta \llbracket \eta \rrbracket J-\llbracket \boldsymbol{q} \rrbracket \cdot \boldsymbol{n}\} \mathrm{d} a-\int_{\partial \mathscr{A}(t)} \vartheta \eta^{x} V_{\partial \mathscr{A}}^{m i g} \mathrm{~d} s=0,
\end{aligned}
$$

which leaves the interfacial energy balance

$$
\frac{\mathrm{d}}{\mathrm{d} t} \int_{\mathscr{A}(t)} \varepsilon^{x} \mathrm{~d} a=w(\mathscr{A}(t))+\int_{\mathscr{A}(t)}\{\vartheta \llbracket \eta \rrbracket J-\llbracket \boldsymbol{q} \rrbracket \cdot \boldsymbol{n}\} \mathrm{d} a+\int_{\partial \mathscr{A}(t)} \vartheta \eta^{x} V_{\partial \mathscr{A}}^{m i g} \mathrm{~d} s .
$$

A strictly analogous argument for the entropy imbalance (6.5) leads to the inequality

$$
\begin{aligned}
& \underbrace{\frac{\mathrm{d}}{\mathrm{d} t} \int_{\mathscr{R}^{+}(t)} \varrho \eta \mathrm{d} v-H\left(\mathscr{R}^{+}(t)\right)}_{(\mathrm{I})}+\underbrace{\frac{\mathrm{d}}{\mathrm{d} t} \int_{\mathscr{R}^{-}(t)} \varrho \eta \mathrm{d} v-H\left(\mathscr{R}^{-}(t)\right)}_{(\mathrm{II})} \\
& +\frac{\mathrm{d}}{\mathrm{d} t} \int_{\mathscr{A}(t)} \eta^{x} \mathrm{~d} a-\int_{\mathscr{A}(t)} \frac{\vartheta \llbracket \eta \rrbracket J-\llbracket \boldsymbol{q} \rrbracket \cdot \boldsymbol{n}}{\vartheta}-\int_{\partial \mathscr{A}(t)} \eta^{x} V_{\partial \mathscr{A}}^{m i g} \mathrm{~d} s \geqslant 0 .
\end{aligned}
$$

Using the divergence theorem and the transport identity (3.4), the terms (I) and (II) lead to the inequalities

$$
\int_{\mathscr{R} \pm(t)}\left\{\varrho \dot{\eta}+\operatorname{div} \frac{\boldsymbol{q}}{\vartheta}\right\} \mathrm{d} v \geqslant 0
$$

(cf. $(2.10 b)$ ). Given any migrating subsurface $\mathscr{A}(t)$, we may choose the migrating control volumes $\mathscr{R}^{ \pm}(t)$ to be $\delta$-pillboxes $\mathscr{R}_{\delta}^{ \pm}(t)$ about $\mathscr{A}(t)$ (cf. (5.10)); for this choice, the terms (6.10) tend to zero as $\delta \rightarrow 0$ leaving the interfacial entropy imbalance

$$
\frac{\mathrm{d}}{\mathrm{d} t} \int_{\mathscr{A}(t)} \eta^{x} \mathrm{~d} a \geqslant \int_{\mathscr{A}(t)} \frac{\vartheta \llbracket \eta \rrbracket J-\llbracket \boldsymbol{q} \rrbracket \cdot \boldsymbol{n}}{\vartheta}+\int_{\partial \mathscr{A}(t)} \eta^{x} V_{\partial \mathscr{A}}^{m i g} \mathrm{~d} s .
$$


Step 2. Interfacial Eshelby relation. Pointwise localization

To localize the interfacial laws (6.8) and (6.11) it is necessary, in some sense, to take the time derivatives $(\mathrm{d} / \mathrm{d} t) \int_{\mathscr{A}} \varepsilon^{x} \mathrm{~d} a$ and $(\mathrm{d} / \mathrm{d} t) \int_{\mathscr{A}} \eta^{x} \mathrm{~d} a$ inside the integrals, an operation facilitated by the following result:

Superficial TRANSPORT THEOREM. For $\gamma(\boldsymbol{x}, t)$ a superficial scalar field and $\dot{\gamma}$ its migrationally normal time derivative following $\mathscr{S}$ as defined in (4.31),

$$
\frac{\mathrm{d}}{\mathrm{d} t} \int_{\mathscr{A}} \gamma \mathrm{d} a=\int_{\mathscr{A}}\left\{\dot{\gamma}-\gamma\left(K V-\operatorname{div}_{\mathscr{S}} \boldsymbol{u}_{\text {tan }}\right)\right\} \mathrm{d} a+\int_{\partial \mathscr{A}} \gamma V_{\partial \mathscr{A}}^{m i g} \mathrm{~d} s .
$$

(Cf. Cermelli et al. (2005), who derive (6.12) based on a transport theorem of Gurtin et al. (1989).)

The interfacial energy balance (6.8) with $w(\mathscr{A})$ by (5.30) and $(\mathrm{d} / \mathrm{d} t) \int_{\mathscr{A}} \varepsilon^{x} \mathrm{~d} a$ given by (6.12) with $\gamma=\varepsilon^{x}$ has the form

$$
\begin{aligned}
\int_{\mathscr{A}(t)}\left\{\stackrel{\circ}{\varepsilon}^{x}-\varepsilon^{x}\left(K V-\operatorname{div}_{\mathscr{S}} \boldsymbol{u}_{t a n}\right)\right\} \mathrm{d} a \\
=-\int_{\mathscr{A}(t)}\left\{\varphi\left(K V-\operatorname{div}_{\mathscr{S}} \boldsymbol{u}_{t a n}\right)+\mathbb{C}_{t a n}: \mathbb{D}-\boldsymbol{c} \cdot \operatorname{grad}_{\mathscr{S}} V^{m i g}+F J\right\} \mathrm{d} a, \\
\quad+\int_{\mathscr{A}(t)}\{\vartheta \llbracket \eta \rrbracket J-\llbracket \boldsymbol{q} \rrbracket \cdot \boldsymbol{n}\} \mathrm{d} a+\int_{\partial \mathscr{A}(t)}\left\{\varphi-\varepsilon^{x}+\vartheta \eta^{x}\right\} V_{\partial \mathscr{A}}^{m i g} \mathrm{~d} s .
\end{aligned}
$$

Consider the underlined term, which is the sole term involving an integral over $\partial \mathscr{A}$. Since the migrating subsurface $\mathscr{A}(t)$ is arbitrary, we may, at any given time, vary $V_{\partial \mathscr{A}}$ and (hence) $V_{\partial \mathscr{A}}^{m i g}=V_{\partial \mathscr{A}}-\boldsymbol{u}_{t a n} \cdot \boldsymbol{v}_{\partial \mathscr{A}}$ arbitrarily without changing the remaining fields involved in (6.13). Thus for (6.13) to be valid for all choices of the migrating subsurface $\mathscr{A}(t)$, we must have

$$
\varphi=\varepsilon^{x}-\vartheta \eta^{x}=\psi^{x},
$$

and (5.25) takes the form of an interfacial Eshelby relation

$$
\mathbb{C}_{t a n}=\psi^{x} \mathbb{P}-\mathbb{T} \text {. }
$$

The chief difference between (6.15) and its bulk counterpart (3.16) is the absence of a kinetic-energy term in (6.15), as brought about by our neglect of interfacial mass.

Next, if we use (6.14) to rewrite (6.13) as

$$
\begin{aligned}
\int_{\mathscr{A}(t)} & \left\{\stackrel{\circ}{\varepsilon}{ }^{x}-\vartheta \eta^{x}\left(K V-\operatorname{div}_{\mathscr{S}} \boldsymbol{u}_{t a n}\right)\right\} \mathrm{d} a \\
= & -\int_{\mathscr{A}(t)}\left\{\llbracket \boldsymbol{q} \rrbracket \cdot \boldsymbol{n}+\mathbb{C}_{t a n}: \mathbb{D}-\boldsymbol{c} \cdot \operatorname{grad}_{\mathscr{S}} V^{m i g}-(\vartheta \llbracket \eta \rrbracket-F) J\right\} \mathrm{d} a,
\end{aligned}
$$

then, since $\mathscr{A}$ is arbitrary, we are led to the local form of the energy balance:

$$
\stackrel{\circ}{\varepsilon}^{x}=\vartheta \eta^{x}\left(K V-\operatorname{div}_{\mathscr{s}} \boldsymbol{u}_{t a n}\right)-\llbracket \boldsymbol{q} \rrbracket \cdot \boldsymbol{n}+\vartheta \llbracket \eta \rrbracket J-\mathbb{C}_{t a n}: \mathbb{D}+\boldsymbol{c} \cdot \operatorname{grad}_{\mathscr{s}} V^{m i g}-F J .
$$

Similarly, by (6.11) and (6.12) with $\gamma=\eta^{x}$, we have the local form of the entropy imbalance:

$$
\vartheta \stackrel{\circ}{\eta}^{x} \geqslant \vartheta \eta^{x}\left(K V-\operatorname{div}_{s} \boldsymbol{u}_{t a n}\right)-\llbracket \boldsymbol{q} \rrbracket \cdot \boldsymbol{n}+\vartheta \llbracket \eta \rrbracket J
$$

Let

$$
f^{*} \stackrel{\text { def }}{=}\langle\langle v\rangle\rangle^{-1} F
$$


so that, by (5.28) and (5.31),

$$
F J=f^{*} V^{m i g}, \quad f^{*}=f-\frac{\llbracket v \rrbracket}{\langle v\rangle}\{\boldsymbol{n} \cdot\langle\langle\boldsymbol{C}\rangle\rangle \boldsymbol{n}-J\langle\langle\boldsymbol{u}\rangle \cdot \boldsymbol{n}\} .
$$

Then, subtracting (6.17) from (6.16) and using (6.2), we arrive at the interfacial dissipation inequality

$$
\stackrel{\circ}{\psi}^{x}+\eta^{x} \stackrel{\circ}{\vartheta}+\mathbb{C}_{t a n}: \mathbb{D}-c \cdot \operatorname{grad}_{\mathscr{S}} V^{m i g}+f^{*} V^{m i g} \leqslant 0 .
$$

This inequality is basic to our discussion of constitutive equations. As we shall see, the field $f^{*}$ represents the dissipative part of the normal internal force $f$.

Remark (i). The standard surface stress $\mathbb{T}$ does not appear in the interfacial dissipation inequality (6.20); for that reason, we consider $\mathbb{T}$ to be constitutively indeterminate. On the other hand, the term $\mathbb{C}_{\text {tan }}: \mathbb{D}$ in (6.20) would imply the need for a constitutive relation for $\mathbb{C}_{t a n}$, which, with the interfacial Eshelby relation in the form $\mathbb{T}=\psi^{x} \mathbb{P}-\mathbb{C}_{\text {tan }}$ would yield an auxiliary relation for $\mathbb{T}$ (cf. (9.1)).

Remark (ii). Similarly, by (6.19), $\mathbb{P} \boldsymbol{f}^{x}-$ the tangential part of the internal configurational force $f^{x}$ for the interface - is not restricted by (6.20). We therefore consider $\mathbb{P} \boldsymbol{f}^{x}$ to be constitutively indeterminate and hence adjustable to ensure satisfaction of the tangential part of the configurational force balance (5.16). The role of $\mathbb{P} \boldsymbol{f}^{x}$ in the theory is analogous to that of the bulk pressure in an incompressible fluid.

Remark (iii). Taking advantage of the bulk and interfacial Eshelby relations (3.16) and (6.15), we may rewrite the energy balance (6.4) for a migrating control volume $\mathscr{R}(t)$ containing a portion $\mathscr{A}(t)$ of the interface $\mathscr{S}(t)$ in a form,

$$
\begin{aligned}
& \frac{\mathrm{d}}{\mathrm{d} t} \int_{\mathscr{R}(t)} \varrho\left\{\varepsilon+\frac{1}{2}|\boldsymbol{u}|^{2}\right\} \mathrm{d} v-\int_{\partial \mathscr{R}(t)}\left\{\varepsilon+\frac{1}{2}|\boldsymbol{u}|^{2}\right\} \boldsymbol{J}_{\partial \mathscr{R}} \mathrm{d} a+\frac{\mathrm{d}}{\mathrm{d} t} \int_{\mathscr{A}(t)} \varepsilon^{x} \mathrm{~d} a-\int_{\partial \mathscr{A}(t)} \varepsilon^{x} V_{\partial \mathscr{A}}^{m i g} \mathrm{~d} s \\
& =\int_{\partial \mathscr{R}(t)} \boldsymbol{T} \boldsymbol{m} \cdot \boldsymbol{u} \mathrm{d} a+\int_{\partial \mathscr{A}(t)} \mathbb{T} \boldsymbol{v}_{\partial \mathscr{A}} \cdot \boldsymbol{u}_{t a n} \mathrm{~d} s-\int_{\partial \mathscr{R}(t)} \boldsymbol{q} \cdot \boldsymbol{m} \mathrm{d} a,
\end{aligned}
$$

involving only standard forces. Additionally, the entropy imbalance (6.5) can be rewritten as

$$
\frac{\mathrm{d}}{\mathrm{d} t} \int_{\mathscr{R}(t)} \varrho \eta \mathrm{d} v-\int_{\partial \mathscr{R}(t)} \eta J_{\partial \mathscr{R}} \mathrm{d} a+\frac{\mathrm{d}}{\mathrm{d} t} \int_{\mathscr{A}(t)} \eta^{x} \mathrm{~d} a-\int_{\partial \mathscr{A}(t)} \eta^{x} V_{\partial \mathscr{A}}^{m i g} \mathrm{~d} s \geqslant-\int_{\partial \mathscr{R}(t)} \frac{\boldsymbol{q}}{\vartheta} \cdot \boldsymbol{m} \mathrm{d} a .
$$

These versions of the first and second laws simply generalize (3.9) to account for flows of energy and entropy across $\partial \mathscr{A}$ as well as the power expended on $\partial \mathscr{A}$ by the standard interfacial traction.

\section{Standard and configurational momentum balances revisited}

7.1. Standard momentum balance

Using the interfacial Eshelby relation (6.15) and (4.7),

$$
\operatorname{div}_{\mathscr{S}} \mathbf{T}=\psi^{x} K \boldsymbol{n}+\operatorname{grad}_{\mathscr{S}} \psi^{x}-\operatorname{div}_{\mathscr{S}} \mathbb{C}_{t a n},
$$

and we may rewrite the standard momentum balance $(5.11 a)$ in the form

$$
\psi^{x} K \boldsymbol{n}+\operatorname{grad}_{\mathscr{S}} \psi^{x}-\operatorname{div}_{\mathscr{S}} \mathbb{C}_{t a n}+\llbracket \boldsymbol{T} \rrbracket \boldsymbol{n}=J^{2} \llbracket v \rrbracket \boldsymbol{n} .
$$




\subsection{Normal configurational momentum balance}

Combining (6.19b) and the normal configurational momentum balance in the form (5.20) and making use of the identity (4.13), we find that

$\left\langle\langle v\rangle \operatorname{div}_{s} \boldsymbol{c}+\left\langle\langle v\rangle \mathbb{C}_{t a n}: \mathbb{K}+\left\langle\langle v\rangle f^{*}+\llbracket v \rrbracket \boldsymbol{n} \cdot\langle\boldsymbol{C} \boldsymbol{n}\rangle+\langle v\rangle\right\rangle \boldsymbol{n} \cdot \llbracket \boldsymbol{C} \rrbracket \boldsymbol{n}=J\{\llbracket v \rrbracket\langle\boldsymbol{u}\rangle \cdot \boldsymbol{n}+\langle\langle v\rangle \rrbracket \llbracket \boldsymbol{u} \rrbracket \cdot \boldsymbol{n}\}\right.\right.$, or, equivalently, by (4.10), that

$$
\langle v\rangle \operatorname{div}_{s} \boldsymbol{c}+\left\langle\langle v\rangle \mathbb{C}_{t a n}: \mathbb{K}+\left\langle\langle v\rangle f^{*}+\llbracket v(\boldsymbol{C} \boldsymbol{n}-\boldsymbol{u} J) \rrbracket \cdot \boldsymbol{n}=0 .\right.\right.
$$

Thus, appealing to the bulk Eshelby relation (3.16) and making use of the identity

$$
\begin{aligned}
\llbracket \frac{1}{2}|\boldsymbol{u}|^{2}+(V-\boldsymbol{u} \cdot \boldsymbol{n}) \boldsymbol{u} \cdot \boldsymbol{n} \rrbracket & =\llbracket \frac{1}{2}(\boldsymbol{u} \cdot \boldsymbol{n})^{2}+(V-\boldsymbol{u} \cdot \boldsymbol{n}) \boldsymbol{u} \cdot \boldsymbol{n} \rrbracket \\
& =\llbracket V \boldsymbol{u} \cdot \boldsymbol{n}-\frac{1}{2}(\boldsymbol{u} \cdot \boldsymbol{n})^{2} \rrbracket \\
& =-\frac{1}{2} \llbracket(V-\boldsymbol{u} \cdot \boldsymbol{n})^{2} \rrbracket \\
& =-\frac{1}{2} J^{2} \llbracket v^{2} \rrbracket,
\end{aligned}
$$

we find that

$$
\langle v\rangle\rangle\left(\operatorname{div}_{s} \boldsymbol{c}+\mathbb{C}_{t a n}: \mathbb{K}+f^{*}\right)+\llbracket \psi \rrbracket-\boldsymbol{n} \cdot \llbracket v \boldsymbol{T} \rrbracket \boldsymbol{n}+\frac{1}{2} J^{2} \llbracket v^{2} \rrbracket=0 .
$$

Reversing the foregoing steps, we see that (7.2) is equivalent to the normal configurational force balance in the form (5.20). The relation (7.2) represents the normal configurational force balance, expressed per unit mass.

\subsection{Normal combined momentum balance}

The interfacial Eshelby relation couples the standard and configurational stresses $\mathbb{T}$ and $\mathbb{C}$, a coupling that allows us to obtain a useful combination of the standard and configurational momentum balances. To begin with, using (5.13) to eliminate the term $J^{2} \llbracket v \rrbracket$ from (5.20) yields a relation,

$$
\operatorname{div}_{\mathscr{s}} \boldsymbol{c}+\left(\mathbb{T}+\mathbb{C}_{\mathrm{tan}}\right): \mathbb{K}+f+\boldsymbol{n} \cdot \llbracket \boldsymbol{T}+\boldsymbol{C} \rrbracket \boldsymbol{n}=0,
$$

which, when combined with the bulk and interfacial Eshelby relations (3.16) and (6.15) and the identity $\mathbb{P}: \mathbb{K}=K$, has the form

$$
\operatorname{div}_{\mathscr{c}} \boldsymbol{c}+\psi^{x} K+f+\llbracket \varrho\left(\psi-\frac{1}{2}|\boldsymbol{u}|^{2}\right) \rrbracket=0 .
$$

Thus, appealing to $(6.19 b)$, the bulk Eshelby relation (3.16), and (4.13) and using (4.10) and (4.15), we see that

$$
\begin{aligned}
f+\llbracket \varrho\left(\psi-\frac{1}{2}|\boldsymbol{u}|^{2}\right) \rrbracket & =f^{*}+\frac{\llbracket v \rrbracket}{\langle\langle v\rangle}\{\boldsymbol{n} \cdot\langle\langle\boldsymbol{C}\rangle\rangle \boldsymbol{n}-J\langle\boldsymbol{u}\rangle \cdot \boldsymbol{n}\}+\llbracket \varrho\left(\psi-\frac{1}{2}|\boldsymbol{u}|^{2}\right) \rrbracket \\
& \left.=f^{*}+\frac{1}{\langle\langle v\rangle}\left\{\llbracket \psi-\frac{1}{2}|\boldsymbol{u}|^{2} \rrbracket+\frac{1}{2} \llbracket(\boldsymbol{u} \cdot \boldsymbol{n})^{2} \rrbracket\right\}-\frac{\llbracket v \rrbracket}{\langle v v\rangle} \boldsymbol{n} \cdot\langle\boldsymbol{T}\rangle\right\rangle \boldsymbol{n}, \\
& =f^{*}+\langle u v\rangle^{-1} \llbracket \psi \rrbracket-\frac{\llbracket v \rrbracket}{\langle\langle v\rangle} \boldsymbol{n} \cdot\langle\langle\boldsymbol{T}\rangle\rangle,
\end{aligned}
$$

and we are led to the normal combined momentum balance

$$
\operatorname{div}_{\mathscr{c}} \boldsymbol{c}+\psi^{x} K+f^{*}+\left\langle\langle v\rangle^{-1} \llbracket \psi \rrbracket-\zeta \boldsymbol{n} \cdot\langle\boldsymbol{T}\rangle\right\rangle \boldsymbol{n}=0,
$$

with

$$
\zeta \stackrel{\text { def }}{=}-\frac{\llbracket \varrho \rrbracket}{\langle\varrho\rangle\rangle}=\frac{\llbracket v \rrbracket}{\langle\langle v\rangle}
$$

a dimensionless measure of the density jump across the interface. 
Granted (7.1), (7.5) implies (5.20) (with $\boldsymbol{C}$ and $\mathbb{C}$ as determined by (3.16) and (6.15)).

\subsection{Complete set of momentum balances}

A complete set of momentum balances for the interface consists of:

(i) the standard momentum balance in the form (7.1); and

(ii) either the normal configurational momentum balance in the form (7.2) or the normal combined balance (7.5).

\section{Interfacial constitutive relations}

Consistent with our treatment of the bulk phases, we suppose that the free energy and entropy of the interface are determined by state relations

$$
\psi^{x}=\hat{\psi}^{x}(\vartheta), \quad \eta^{x}=-\frac{\mathrm{d} \hat{\psi}^{x}(\vartheta)}{\mathrm{d} \vartheta}
$$

Granted this, the interfacial dissipation inequality (6.20) reduces to

$$
\mathscr{D} \stackrel{\text { def }}{=}-\mathbb{C}_{t a n}: \mathbb{D}+\boldsymbol{c} \cdot \operatorname{grad}_{\mathscr{S}} V^{m i g}-f^{*} V^{m i g} \geqslant 0,
$$

with $\mathscr{D}$ the interfacial dissipation, per unit area; we refer to (8.2) as the dissipation inequality.

Guided by the dissipation inequality, we posit isotropic constitutive equations giving $\mathbb{C}_{\text {tan }}, \boldsymbol{c}$ and $f^{*}$ as functions of $\vartheta$, and

$$
\left(\mathbb{D}, V^{m i g}, \operatorname{grad}_{\mathscr{S}} V^{m i g}\right) .
$$

Assuming, for convenience, that these relations are linear and uncoupled in the arguments (8.3), we are led to a kinetic relation

$$
f^{*}=-\kappa(\vartheta) V^{m i g},
$$

with modulus $\kappa(\vartheta)$, and interfacial flow relations

$$
\mathbb{C}_{\text {tan }}=-2 \alpha(\vartheta) \mathbb{D}-\lambda(\vartheta)(\operatorname{tr} \mathbb{D}) \mathbb{P}, \quad \boldsymbol{c}=\beta(\vartheta) \operatorname{grad}_{\mathscr{S}} V^{m i g},
$$

with moduli $\alpha(\vartheta), \lambda(\vartheta)$ and $\beta(\vartheta)$. Here:

(i) $\alpha(\vartheta)$ and $\lambda(\vartheta)$ represent (interfacial) fluid viscosities; $\alpha(\vartheta)$, the shear viscosity, is associated with shearing of the interface, while $\lambda(\vartheta)+\alpha(\vartheta)$, the dilatational viscosity, is associated with changes in the local area of the interface;

(ii) $\kappa(\vartheta)$ and $\beta(\vartheta)$, which represent migrational viscosities, describe dissipative effects in the exchange of atoms between phases.

The constitutive relations (8.3)-(8.5) and the identities (4.3) and (4.19) render the dissipation (8.2) of the form

$$
\mathscr{D}=2 \alpha(\vartheta)\left|\mathbb{D}_{0}\right|^{2}+\{\lambda(\vartheta)+\alpha(\vartheta)\}(\operatorname{tr} \mathbb{D})^{2}+\beta(\vartheta)\left|\operatorname{grad}_{\mathscr{S}} V^{m i g}\right|^{2}+\kappa(\vartheta)\left|V^{m i g}\right|^{2},
$$

with

$$
\mathbb{D}_{0}=\mathbb{D}-\frac{1}{2}(\operatorname{tr} \mathbb{D}) \mathbb{P}
$$

the deviatoric stretch rate. Thus, since the tensor fields $\operatorname{tr} \mathbb{D}$ and $\mathbb{D}_{0}$ may be specified independently, the dissipation inequality (8.2) is satisfied if and only if the moduli satisfy

$$
\alpha(\vartheta) \geqslant 0, \quad \lambda(\vartheta)+\alpha(\vartheta) \geqslant 0, \quad \kappa(\vartheta) \geqslant 0, \quad \beta(\vartheta) \geqslant 0 .
$$


In particular, the interface is inviscid (and hence dissipationless) if and only if

$$
\alpha(\vartheta)=\lambda(\vartheta)=\kappa(\vartheta)=\beta(\vartheta)=0,
$$

in which case both the configurational stress $\mathbb{C}$ and the dissipative part $f^{*}$ of the normal internal force $f$ vanish. Thus, in particular, interfacial configurational stress is not present at an inviscid interface.

Remark. The most general linear, isotropic constitutive equations consist of coupled relations

$$
\begin{aligned}
\operatorname{tr} \mathbb{C}_{t a n} & =-2\{\lambda(\vartheta)+\alpha(\vartheta)\} \operatorname{tr} \mathbb{D}-\bar{\zeta}(\vartheta) V^{m i g}, \\
f^{*} & =-\zeta(\vartheta) \operatorname{tr} \mathbb{D}-\kappa(\vartheta) V^{m i g},
\end{aligned}
$$

together with uncoupled relations $\left(\mathbb{C}_{t a n}\right)_{0}=-\alpha(\vartheta) \mathbb{D}_{0}$ and $c=\beta(\vartheta) \operatorname{grad}_{\mathscr{c}} V^{\text {mig }}$, where $\left(\mathbb{C}_{t a n}\right)_{0}$ is the deviatoric part of $\mathbb{C}_{\text {tan }}$. Here, $\bar{\zeta}(\vartheta)$ and $\zeta(\vartheta)$ are constitutive moduli that couple the relations for $\operatorname{tr} \mathbb{C}_{\text {tan }}$ and $f^{*}$.

\section{Consequences of the interfacial constitutive relations}

\subsection{Constitutive relation for the standard interfacial stress}

An interesting and important consequence of the interfacial flow relation $(8.5 a)$ supplemented by the interfacial Eshelby relation (6.15) is an auxiliary constitutive relation for the standard interfacial stress:

$$
\mathbb{T}=\hat{\psi}^{x}(\vartheta) \mathbb{P}+2 \alpha(\vartheta) \mathbb{D}+\lambda(\vartheta)(\operatorname{tr} \mathbb{D}) \mathbb{P} .
$$

Remark (i). By (9.1), the surface tension

$$
\sigma \stackrel{\text { def }}{=} \frac{1}{2} \operatorname{tr} \mathbb{T}=\hat{\psi}^{x}(\vartheta)+\{\lambda(\vartheta)+\alpha(\vartheta)\} \operatorname{tr} \mathbb{D}
$$

is a sum of energetic and viscous terms.

Remark (ii). The superficial stretch-rate $\mathbb{D}$ may be written in the alternative form

$$
\mathbb{D}=\frac{1}{2}\left\{\mathbb{P g r a d}_{s} \boldsymbol{u}_{t a n}+\left(\operatorname{grad}_{s} \boldsymbol{u}_{t a n}\right)^{\top} \mathbb{P}\right\}-\langle\langle\boldsymbol{u} \cdot \boldsymbol{n}\rangle \mathbb{K} .
$$

In view of $(9.1)$, if the interface $\mathscr{S}$ is material - so that, necessarily, $\boldsymbol{u}$ is continuous across $\mathscr{S}$ - then (9.1) with $\hat{\psi}^{x} \equiv 0$ reduces to an expression due to Scriven (1960).

\subsection{Interfacial energy balance}

As with the bulk phases, the relation $\psi^{x}=\varepsilon^{x}-\vartheta \eta^{x}$ and the state relations (8.1) yield an auxiliary state relation $\varepsilon^{x}=\hat{\varepsilon}^{x}(\vartheta)$, with the consequence that

$$
\frac{\mathrm{d} \hat{\varepsilon}^{x}(\vartheta)}{\mathrm{d} \vartheta}=-\vartheta \frac{\mathrm{d}^{2} \hat{\psi}^{x}(\vartheta)}{\mathrm{d} \vartheta^{2}}=\vartheta \frac{\mathrm{d} \hat{\eta}^{x}(\vartheta)}{\mathrm{d} \vartheta} .
$$

Thus, bearing in mind (6.14), the energy balance (6.16) becomes

$$
\llbracket \boldsymbol{q} \rrbracket \cdot \boldsymbol{n}-\vartheta \llbracket \eta \rrbracket J=-\vartheta\left\{\stackrel{\circ}{\eta}^{x}-\eta^{x}\left(K V-\operatorname{div}_{g} \boldsymbol{u}_{t a n}\right)\right\}+\mathscr{D},
$$

with $\mathscr{D}$ the dissipation (8.6).

\subsection{Standard momentum balance}

The standard momentum balance in the form (7.1) and the constitutive relations (8.1) and $(8.5 a)$ for $\psi^{x}$ and $\mathbb{C}$ yields the balance

$$
\llbracket \boldsymbol{T} \rrbracket \boldsymbol{n}-J^{2} \llbracket v \rrbracket \boldsymbol{n}=-\hat{\psi}^{x}(\vartheta) K \boldsymbol{n}-\operatorname{grad}_{\mathscr{S}} \hat{\psi}^{x}(\vartheta)-\operatorname{div}_{\mathscr{S}}\{2 \alpha(\vartheta) \mathbb{D}+\lambda(\vartheta)(\operatorname{tr} \mathbb{D}) \mathbb{P}\} .
$$


Remark. By (4.9), the normal part of the balance (9.3), namely

$$
\boldsymbol{n} \cdot \llbracket \boldsymbol{T} \rrbracket \boldsymbol{n}-J^{2} \llbracket v \rrbracket=-\hat{\psi}^{x}(\vartheta) K-2 \alpha(\vartheta) \mathbb{K}: \mathbb{D}-\lambda(\vartheta)(\operatorname{tr} \mathbb{D}) K,
$$

represents a generalization of the classical Young-Laplace equation (Young 1805; Laplace 1806) to account for the inertia of the bulk phases and interfacial viscosity. Note the presence of the full curvature tensor $\mathbb{K}$.

\subsection{Normal configurational momentum balance}

The normal configurational momentum balance, expressed per unit mass as in (7.2), augmented by the constitutive relations (8.4) and (8.5), yields the balance

$$
\begin{aligned}
& \llbracket \psi \rrbracket-\boldsymbol{n} \cdot \llbracket v \boldsymbol{T} \rrbracket \boldsymbol{n}+\frac{1}{2} J^{2} \llbracket v^{2} \rrbracket \\
& =\langle u\rangle\left\{\kappa(\vartheta) V^{m i g}-\operatorname{div}_{\mathscr{S}}\left(\beta(\vartheta) \operatorname{grad}_{\mathscr{S}} V^{m i g}\right)+2 \alpha(\vartheta) \mathbb{K}: \mathbb{D}+\lambda(\vartheta)(\operatorname{tr} \mathbb{D}) K\right\} .
\end{aligned}
$$

The balance (9.4) depends on the bulk flow via the migrational velocity $V^{m i g}$ as defined in (4.14).

Remark (i). When inertial effects along with the migrational viscosity $\beta$ and the interfacial fluid viscosities $\alpha$ and $\lambda$ are negligible, and when $\boldsymbol{n} \cdot \llbracket v \boldsymbol{T} \rrbracket \boldsymbol{n}=0,(9.4)$ becomes

$$
\langle v\rangle \kappa_{(\vartheta)} V^{m i g}=\llbracket \psi \rrbracket,
$$

which has the form of an evolution equation due to Frank (1958) - the differences being in the dependence of $\kappa$ on the temperature field and a velocity measured relative to the average fluid velocity at the interface.

Remark (ii). An important consequence of (9.4) is that, if inertial effects are negligible and the interface is inviscid in the sense of (8.7), then

$$
\llbracket \psi \rrbracket=\boldsymbol{n} \cdot \llbracket v \boldsymbol{T} \rrbracket \boldsymbol{n} .
$$

When the bulk stress is a pressure, so that $\boldsymbol{T}=-p \mathbf{1}$, this equation reduces to the classical condition $\llbracket \psi+p v \rrbracket=0$ underlying the Maxwell (1875) equal-area rule for the equilibrium of two fluid phases. In this sense, the condition $\llbracket \psi \rrbracket=\boldsymbol{n} \cdot \llbracket v \boldsymbol{T} \rrbracket \boldsymbol{n}$ can be viewed as a generalization of the equal area rule to account for the viscosities of the bulk phases. Moreover, the general normal configurational balance, (9.4), extends that generalization to account for the migrational and fluid viscosities of the interface.

Remark (iii). When the migrational viscosity $\beta$ and the interfacial viscosities $\alpha$ and $\lambda$ are negligible, we may use the identity $J^{2} \llbracket v^{2} \rrbracket=\llbracket(V-\boldsymbol{u} \cdot \boldsymbol{n})^{2} \rrbracket$ to write (9.4) as

$$
\left\langle\langle v\rangle \kappa(\vartheta) V^{m i g}=\llbracket \psi+\frac{1}{2}(V-\boldsymbol{u} \cdot \boldsymbol{n})^{2}-v \boldsymbol{n} \cdot \boldsymbol{T n} \rrbracket,\right.
$$

which has the form of an equation proposed by Fried (1995).

\subsection{Normal combined momentum balance}

When supplemented by the constitutive relation for $\psi^{x}$ and the kinetic relation (8.4), the normal combined momentum balance (7.5) takes the form

$$
\llbracket \psi \rrbracket-\llbracket v \rrbracket \boldsymbol{n} \cdot\langle\boldsymbol{T}\rangle\rangle \boldsymbol{n}=-\left\langle\langle v\rangle \hat{\psi}^{x}(\vartheta) K+\left\langle\langle v \rangle \left\langle\left\{\kappa(\vartheta) V^{m i g}-\operatorname{div}_{\mathscr{S}}\left(\beta(\vartheta) \operatorname{grad}_{\mathscr{S}} V^{m i g}\right)\right\} .\right.\right.\right.
$$

Note that this equation is independent of the interfacial fluid viscosities $\alpha$ and $\lambda$.

Remark. When the migrational viscosity $\beta$ is negligible and the two phases have the same density $(\zeta=0),(9.5)$ becomes

$$
\hat{\psi}^{x}(\vartheta) K=\kappa(\vartheta) V^{m i g}-\left\langle\langle v\rangle^{-1} \llbracket \psi \rrbracket,\right.
$$


an equation that is independent of bulk stress and reminiscent of the classical curvature-flow equation (Mullins 1956) driven by a difference in bulk free-energies (Angenent \& Gurtin 1989).

\section{Summary of general interface conditions}

Apart from appropriate kinematical equations, the basic equations for the interface therefore consist of the balances (6.16), (7.1) and (7.2), for energy, standard momentum and normal configurational momentum augmented by the interfacial Eshelby relation (6.15) and the interfacial constitutive relations (8.1), (8.4) and (8.5). These equations combine as shown in $\S 9$ to form basic interface conditions consisting of the energy balance

$$
\llbracket \boldsymbol{q} \rrbracket \cdot \boldsymbol{n}-\vartheta \llbracket \eta \rrbracket J=-\vartheta\left\{\stackrel{\circ}{\eta}^{x}-\eta^{x}\left(K V-\operatorname{div}_{\mathscr{S}} \boldsymbol{u}_{t a n}\right)\right\}+\mathscr{D},
$$

with $\eta^{x}=-\mathrm{d} \psi^{x}(\vartheta) / \mathrm{d} \vartheta$ and $\mathscr{D}$ given by (8.6) and where $\stackrel{\circ}{ }^{x}$ is related to the (standard) normal time derivative $\stackrel{\square}{\eta}^{x}$ following $\mathscr{S}$ through the relation $\stackrel{\circ}{\eta}^{x}=\stackrel{\square}{\eta}^{x}+\boldsymbol{u}_{\text {tan }} \cdot \operatorname{grad}_{\mathscr{f}} \eta^{x}$ (as noted in $\S 4.7$ ), the standard momentum balance

$$
\llbracket \boldsymbol{T} \rrbracket \boldsymbol{n}-J^{2} \llbracket v \rrbracket \boldsymbol{n}=-\hat{\psi}^{x}(\vartheta) K \boldsymbol{n}-\operatorname{grad}_{\mathscr{S}} \hat{\psi}^{x}(\vartheta)-\operatorname{div}_{\mathscr{S}}\{2 \alpha(\vartheta) \mathbb{D}+\lambda(\vartheta)(\operatorname{tr} \mathbb{D}) \mathbb{P}\},
$$

and either the normal configurational momentum balance

$$
\begin{aligned}
& \llbracket \psi \rrbracket-\boldsymbol{n} \cdot \llbracket v \boldsymbol{T} \rrbracket \boldsymbol{n}+\frac{1}{2} J^{2} \llbracket v^{2} \rrbracket \\
& =\left\langle\langle v\rangle\left\{\kappa(\vartheta) V^{m i g}-\operatorname{div}_{\mathscr{S}}\left(\beta(\vartheta) \operatorname{grad}_{\mathscr{S}} V^{m i g}\right)+2 \alpha(\vartheta) \mathbb{K}: \mathbb{D}+\lambda(\vartheta)(\operatorname{tr} \mathbb{D}) K\right\}\right.
\end{aligned}
$$

or the normal combined force balance

$$
\llbracket \psi \rrbracket-\llbracket v \rrbracket \boldsymbol{n} \cdot\langle\langle\boldsymbol{T}\rangle\rangle \boldsymbol{n}=-\left\langle\langle v\rangle \hat{\psi}^{x}(\vartheta) K+\langle\langle v\rangle\rangle\left\{\kappa(\vartheta) V^{m i g}-\operatorname{div}_{\mathscr{S}}\left(\beta(\vartheta) \operatorname{grad}_{\mathscr{S}} V^{m i g}\right)\right\} .\right.
$$

\section{Existence of an equilibrium temperature}

\subsection{Latent heat. Free-energy theorem}

If the interface is flat and at rest, and the bulk fluid is unstressed ( $\boldsymbol{T}=\mathbf{0})$, then the normal combined balance implies that

$$
\llbracket \psi \rrbracket=0,
$$

a condition that represents a thermal equilibrium. Since the bulk free-energy depends on temperature, (11.1) is, in essence, a condition on the interfacial temperature. With this as background, we now add the following constitutive assumption:

(C1) there is a unique (constant) equilibrium temperature $\vartheta_{0}$ such that

$$
\psi^{+}\left(\vartheta_{0}\right)=\psi^{-}\left(\vartheta_{0}\right), \quad \varepsilon^{+}\left(\vartheta_{0}\right) \neq \varepsilon^{-}\left(\vartheta_{0}\right) .
$$

In what follows a subscripted zero denotes evaluation at $\vartheta_{0}$. The scalar constant $\ell=\llbracket \varepsilon_{0} \rrbracket$ represents the latent heat at the temperature $\vartheta_{0}$; by (11.2),

$$
\ell=\vartheta_{0} \llbracket \eta_{0} \rrbracket, \quad \llbracket \eta_{0} \rrbracket \neq 0 .
$$

The next theorem is central to what follows. In stating this result, it is useful to define a dimensionless temperature $\theta$ by

$$
\theta=\frac{\vartheta-\vartheta_{0}}{\vartheta_{0}}
$$


FreE-EnERGY THEOREM

(I) If the bulk specific heat is independent of phase, then the state fields obey the simple jump relations

$$
\llbracket \psi \rrbracket=-\ell \theta, \quad \llbracket \eta \rrbracket \equiv \frac{\ell}{\vartheta_{0}}, \quad \llbracket \varepsilon \rrbracket \equiv \ell,
$$

and conversely.

(II) The jumps in free energy and entropy obey the following estimates:

$$
\llbracket \psi \rrbracket=-\ell \theta+\mathrm{O}\left(\theta^{2}\right), \quad \llbracket \eta \rrbracket=\frac{\ell}{\vartheta_{0}}+\mathrm{O}(\theta) .
$$

To verify (I), assume first that

$$
\frac{\mathrm{d} \varepsilon^{+}}{\mathrm{d} \vartheta}=\frac{\mathrm{d} \varepsilon^{-}}{\mathrm{d} \vartheta}
$$

so that $\llbracket \varepsilon \rrbracket \equiv \llbracket \varepsilon_{0} \rrbracket$. By (2.13), the jumps $\llbracket \psi \rrbracket$, $\llbracket \eta \rrbracket$ and $\llbracket \varepsilon \rrbracket$, considered as functions of $\vartheta$, obey the thermodynamic relations

$$
\llbracket \psi \rrbracket=\llbracket \varepsilon \rrbracket-\vartheta \llbracket \eta \rrbracket, \quad \llbracket \eta \rrbracket=-\frac{\mathrm{d} \llbracket \psi \rrbracket}{\mathrm{d} \vartheta},
$$

and these relations, the condition $\llbracket \varepsilon \rrbracket \equiv \llbracket \varepsilon_{0} \rrbracket$, and (11.4) yield (11.5). Conversely, (11.5) implies that $\llbracket \varepsilon \rrbracket \equiv \llbracket \varepsilon_{0} \rrbracket$ and hence that (11.7) is satisfied. Thus (I) is valid.

Consider (II). Expanding $G(\vartheta)=\psi^{+}(\vartheta)-\psi^{-}(\vartheta)$ about $\vartheta=\vartheta_{0}$, we find that, by (11.8),

$$
G(\vartheta)=-\llbracket \eta_{0} \rrbracket\left(\vartheta-\vartheta_{0}\right)+O\left(\theta^{2}\right), \quad \frac{\mathrm{d} G(\vartheta)}{\mathrm{d} \vartheta}=O(1)
$$

thus, using (11.3), we arrive at the estimates (11.6). This completes the proof of the free-energy theorem.

\subsection{Estimates for the temperature}

A direct consequence of (10.3) and (10.4) and the free-energy theorem are the following estimates for the temperature:

$$
\begin{aligned}
\ell \theta= & -\boldsymbol{n} \cdot \llbracket v \boldsymbol{T} \rrbracket \boldsymbol{n}+\frac{1}{2} J^{2} \llbracket v^{2} \rrbracket-\left\langle\langle v \rangle \left\{\kappa(\vartheta) V^{m i g}-\operatorname{div}_{\mathscr{S}}\left(\beta(\vartheta) \operatorname{grad}_{\mathscr{S}} V^{m i g}\right)\right.\right. \\
& +2 \alpha(\vartheta) \mathbb{K}: \mathbb{D}+\lambda(\vartheta)(\operatorname{tr} \mathbb{D}) K\}+O\left(\theta^{2}\right), \\
\ell \theta= & -\llbracket v \rrbracket \boldsymbol{n} \cdot\left\langle\langle\boldsymbol{T}\rangle \boldsymbol{n}+\left\langle\langle v\rangle \hat{\psi}^{x}(\vartheta) K-\langle\langle v\rangle\right.\right. \\
& \times\left\{\kappa(\vartheta) V^{m i g}-\operatorname{div}_{\mathscr{S}}\left(\beta(\vartheta) \operatorname{grad}_{\mathscr{S}} V^{m i g}\right)\right\}+\mathrm{O}\left(\theta^{2}\right) .
\end{aligned}
$$

Equations (11.9) represent respective estimates for the normal configurational and normal combined momentum balances. If the interface is inviscid in the sense of (8.7), then (11.9) become

$$
\begin{aligned}
& \ell \theta=-\boldsymbol{n} \cdot \llbracket v \boldsymbol{T} \rrbracket \boldsymbol{n}+\frac{1}{2} J^{2} \llbracket v^{2} \rrbracket+O\left(\theta^{2}\right), \\
& \ell \theta=-\llbracket v \rrbracket \boldsymbol{n} \cdot\langle\langle\boldsymbol{T}\rangle\rangle \boldsymbol{n}+\left\langle\langle v\rangle \hat{\psi}^{x}(\vartheta) K+O\left(\theta^{2}\right) .\right.
\end{aligned}
$$

Finally, if the bulk specific heat is independent of phase, then the term $O\left(\theta^{2}\right)$ may be dropped from (11.9) and (11.10).

Remark (i). Granted the standard momentum balance (10.2), the two estimates in (11.9) are equivalent, as are the two estimates in (11.10).

Remark (ii). When $\llbracket v \rrbracket=0,(11.10 b)$ yields the classical Gibbs-Thomson equation, (1.3). 


\section{Conditions at an interface whose free energy and viscosities are constant}

The general equations (10.1)-(10.4) expressing energy balance, standard momentum balance, normal configurational momentum balance, and normal combined momentum balance on the interface are complicated. We now consider a simplification afforded by $(\mathrm{C} 1)$ and the following additional constitutive assumptions:

(C2) the interfacial fluid and migrational viscosities $\alpha, \lambda, \kappa$, and $\beta$ are constant.

(C3) the interfacial free energy $\psi^{x}$ is constant, so that $\eta^{x}=0$ and $\varepsilon^{x}=\psi^{x}$.

Assumption (C3) reduces the energy balance (10.1) to

$$
\llbracket q \rrbracket \cdot \boldsymbol{n}-\vartheta \llbracket \eta \rrbracket J=\mathscr{D} .
$$

By (8.6), the dissipation $\mathscr{D}$ is quadratic in the interfacial fields $\mathbb{D}, V^{m i g}$ and $\operatorname{grad}_{\mathscr{S}} V^{m i g}$, and it would seem reasonable to limit our discussion to situations in which this dissipation is small compared to the remaining terms in (12.1), which involve only bulk fields. Therefore,

(C4) we neglect the term $\mathscr{D}$ in the energy balance (12.1).

Granted (C2)-(C4) and using (7.6), the interface conditions discussed in $\S 10$ take the form

$$
\begin{gathered}
\llbracket \boldsymbol{q} \rrbracket \cdot \boldsymbol{n}=\vartheta \llbracket \eta \rrbracket J, \\
\llbracket \boldsymbol{T} \rrbracket \boldsymbol{n}-J^{2} \llbracket v \rrbracket \boldsymbol{n}=-\psi^{x} K \boldsymbol{n}-\operatorname{div}_{\mathscr{S}}\{2 \alpha \mathbb{D}+\lambda(\operatorname{tr} \mathbb{D}) \mathbb{P}\},
\end{gathered}
$$

and either

$$
\llbracket \psi \rrbracket-\boldsymbol{n} \cdot \llbracket v \boldsymbol{T} \rrbracket \boldsymbol{n}+\frac{1}{2} J^{2} \llbracket v^{2} \rrbracket=\langle v\rangle\left\langle\left\{V^{m i g}-\beta \Delta_{\mathscr{S}} V^{m i g}+2 \alpha \mathbb{K}: \mathbb{D}+\lambda(\operatorname{tr} \mathbb{D}) K\right\}\right.
$$

or

$$
\llbracket \psi \rrbracket-\llbracket v \rrbracket \boldsymbol{n} \cdot\langle\langle\boldsymbol{T}\rangle\rangle \boldsymbol{n}=-\langle\langle v\rangle\rangle \psi^{x} K+\langle\langle v\rangle\rangle\left\{\kappa V^{m i g}-\beta \Delta_{\mathscr{S}} V^{m i g}\right\} .
$$

Here $\Delta_{\mathscr{S}}$, the Laplace-Beltrami operator, is defined on any superficial field $\varphi$ by

$$
\Delta_{\mathscr{S}} \varphi=\operatorname{div}_{\mathscr{S}}\left(\operatorname{grad}_{\mathscr{S}} \varphi\right)
$$

The next three subsections greatly simplify these interface conditions.

\subsection{Inviscid interface}

The equations are further simplified if we replace $(\mathrm{C} 4)$ by the constitutive assumption

$(\mathrm{C} 4)^{*} \kappa=\alpha=\lambda=\beta=0$ (so that the interface is inviscid).

(By $(8.6),(\mathrm{C} 4)^{*}$ is stronger than $(\mathrm{C} 4)$.) Then the interface conditions (12.2)-(12.4) become

and either

$$
\llbracket \boldsymbol{q} \rrbracket \cdot \boldsymbol{n}=\vartheta \llbracket \eta \rrbracket J, \quad \llbracket \boldsymbol{T} \rrbracket \boldsymbol{n}-J^{2} \llbracket v \rrbracket \boldsymbol{n}=-\psi^{x} K \boldsymbol{n},
$$

$$
\llbracket \psi \rrbracket-\boldsymbol{n} \cdot \llbracket v \boldsymbol{T} \rrbracket \boldsymbol{n}+\frac{1}{2} J^{2} \llbracket v^{2} \rrbracket=0
$$

or

$$
\llbracket \psi \rrbracket-\llbracket v \rrbracket \boldsymbol{n} \cdot\langle\langle\boldsymbol{T}\rangle\rangle \boldsymbol{n}=-\langle\langle v\rangle\rangle \psi^{x} K .
$$

12.2. Bulk specific heats independent of phase

If, in addition to $(\mathrm{C} 1)-(\mathrm{C} 4)$, we assume that the bulk specific heats are independent of phase, then, by (11.5), the balance $(12.6 a)$ for energy becomes

$$
\llbracket \boldsymbol{q} \cdot \boldsymbol{n}=\frac{\ell \vartheta}{\vartheta_{0}} J,
$$


the standard force balance remains (12.6b), and the normal configurational and normal combined balances - which are equivalent, granted (12.6b) - may be written in the form (11.9) with the arguments $(\vartheta)$ removed and with the $O\left(\theta^{2}\right)$ terms omitted.

If we further assume that the interface is inviscid, then the interface conditions are

$$
\llbracket \boldsymbol{q} \rrbracket \cdot \boldsymbol{n}=\frac{\ell \vartheta}{\vartheta_{0}} J, \quad \llbracket \boldsymbol{T} \rrbracket \boldsymbol{n}-J^{2} \llbracket v \rrbracket \boldsymbol{n}=-\psi^{x} K \boldsymbol{n},
$$

and either

$$
\ell \theta=-\boldsymbol{n} \cdot \llbracket v \boldsymbol{T} \rrbracket \boldsymbol{n}+\frac{1}{2} J^{2} \llbracket v^{2} \rrbracket
$$

or

$$
\ell \theta=-\llbracket v \rrbracket \boldsymbol{n} \cdot\langle\langle\boldsymbol{T}\rangle\rangle \boldsymbol{n}+\langle\langle v\rangle\rangle \psi^{x} K
$$

Remark. The hypotheses $(\mathrm{C} 1)-(\mathrm{C} 3)$ and $(\mathrm{C} 4)^{*}$ represent additional constitutive assumptions consistent with the constitutive frameworks set out in $\S \S 2.4$ and 9.2. For that reason the simplified theory based on $(\mathrm{C} 1)-(\mathrm{C} 3)$ and $(\mathrm{C} 4)^{*}$ and resulting in the interface conditions (12.10)-(12.12) is consistent with the standard and configurational force balances and the first two laws as discussed in $\S \S 5$ and 6 . In this sense, the interface conditions (12.10)-(12.12) are exact. (Of course, to be exact when coupled to the bulk equations, the specific heats of the two phases must coincide.)

\subsection{Temperature close to its equilibrium value}

If, in addition to (C1)-(C4), we assume that the temperature is close to its equilibrium value and, consequently, consider: (i) the energy balance (12.2a) with $\vartheta$ replaced by $\vartheta_{0}$ in conjunction with (11.6b) neglecting the $O(\theta)$ term; and (ii) the normal configurational and normal combined momentum balances in the form (11.9), neglecting the $O\left(\theta^{2}\right)$ term. The resulting interface conditions then consist of the balances

$$
\begin{gathered}
\llbracket \boldsymbol{q} \rrbracket \cdot \boldsymbol{n}=\ell J, \\
\llbracket \boldsymbol{T} \rrbracket \boldsymbol{n}-J^{2} \llbracket v \rrbracket \boldsymbol{n}=-\psi^{x} K \boldsymbol{n}-\operatorname{div}_{\mathscr{S}}\{2 \alpha \mathbb{D}+\lambda(\operatorname{tr} \mathbb{D}) \mathbb{P}\},
\end{gathered}
$$

and either

$$
\ell \theta=-\boldsymbol{n} \cdot \llbracket v \boldsymbol{T} \rrbracket \boldsymbol{n}+\frac{1}{2} J^{2} \llbracket v^{2} \rrbracket-\left\langle\langle v\rangle\left\{\kappa V^{m i g}-\beta \Delta_{\mathscr{\varphi}} V^{m i g}\right)+2 \alpha \mathbb{K}: \mathbb{D}+\lambda(\operatorname{tr} \mathbb{D}) K\right\}
$$

or

$$
\ell \theta=-\llbracket v \rrbracket \boldsymbol{n} \cdot\langle\langle\boldsymbol{T}\rangle\rangle \boldsymbol{n}+\langle\langle v\rangle\rangle \psi^{x} K-\left\langle\langle v \rangle \left\{\left\langle\kappa V^{m i g}-\beta \Delta_{\mathscr{\varphi}} V^{m i g}\right\} .\right.\right.
$$

The approximate equations (12.13) and (12.14) reduce further when the interface is inviscid to give

$$
\llbracket q \rrbracket \cdot n=\ell J, \quad \llbracket \boldsymbol{T} \rrbracket \boldsymbol{n}-J^{2} \llbracket v \rrbracket n=-\psi^{x} K \boldsymbol{n},
$$

and either

$$
\ell \theta=-\boldsymbol{n} \cdot \llbracket v \boldsymbol{T} \rrbracket \boldsymbol{n}+\frac{1}{2} J^{2} \llbracket v^{2} \rrbracket
$$

or

$$
\ell \theta=\langle u v\rangle \psi^{x} K-\llbracket v \rrbracket \boldsymbol{n} \cdot\langle\langle\boldsymbol{T}\rangle\rangle \boldsymbol{n} .
$$

Remark. Although the assumptions underlying their derivations differ, the sole difference between the interface conditions (12.10)-(12.12) and (12.16)-(12.18) is that the latent heat $\ell$ in (12.16) is replaced by $\ell \vartheta / \vartheta_{0}$ in $(12.10 a)$. 


\section{Application: radial symmetry}

We assume that the interface is a spherical surface with time-dependent radius $R$. The interior of the sphere is the $(-)$ phase; the exterior is the $(+)$ phase. For simplicity, we suppose that the viscosities $\mu^{ \pm}$and conductivities $k^{ \pm}$of the bulk phases are constant (i.e. independent of $\vartheta$ ). We also assume that the specific heats of the bulk phases are constant, so that

$$
\frac{\mathrm{d} \varepsilon^{ \pm}(\vartheta)}{\mathrm{d} \vartheta}=c^{ \pm}
$$

As for the interface, we restrict attention to circumstances in which the temperature is close to its equilibrium value; $\mathrm{cf} . \S 12.3$.

\subsection{Bulk equations}

The basic unknown fields in the bulk phases are the velocity $\boldsymbol{u}$, the pressure $p$, and the temperature $\vartheta$. Writing $r=|\boldsymbol{x}|$ and $\boldsymbol{e}=\boldsymbol{x} / r$, we assume that

$$
\boldsymbol{u}(\boldsymbol{x}, t)=U(r, t) \boldsymbol{e}, \quad p(\boldsymbol{x}, t)=p(r, t), \quad \vartheta(\boldsymbol{x}, t)=\vartheta(r, t) .
$$

In view of (13.2a, computing the gradient of $\boldsymbol{u}$ yields

$$
\boldsymbol{L}=\boldsymbol{D}=\frac{\partial U}{\partial r} \boldsymbol{e} \otimes \boldsymbol{e}+\frac{U}{r}(\mathbf{1}-\boldsymbol{e} \otimes \boldsymbol{e}),
$$

and the constraint $\operatorname{div} \boldsymbol{u}=\operatorname{tr} \boldsymbol{D}=0$ of incompressibility requires that $\partial U / \partial r+2 U / r=$ 0 ; cf. (2.2). Integrating this equation, we find that

$$
U(r, t) \propto \frac{1}{r^{2}},
$$

with the coefficient of proportionality being independent of $r$. For the velocity to be bounded at $\boldsymbol{x}=\mathbf{0}$, the relevant coefficient of proportionality entering (13.4) must vanish in the $(-)$ phase. It therefore follows that

$$
U(r, t)= \begin{cases}0, & r<R(t) \\ \frac{Q(t)}{r^{2}}, & r>R(t)\end{cases}
$$

with $Q$ being an as yet unknown function of time.

By (13.2a) and (13.5), the acceleration has the form

$$
\dot{\boldsymbol{u}}(\boldsymbol{x}, t)= \begin{cases}\mathbf{0}, & 0<r<R(t), \\ \frac{1}{r^{2}}\left(\frac{\mathrm{d} Q(t)}{\mathrm{d} t}-\frac{2 Q^{2}(t)}{r^{3}}\right) \boldsymbol{e}, & R(t)<r<\infty .\end{cases}
$$

Further, by (2.15), (13.3) and (13.5), the extra stress is given by

$$
\boldsymbol{S}(\boldsymbol{x}, t)= \begin{cases}\mathbf{0}, & 0<r<R(t), \\ \frac{2 \mu^{+} Q(t)}{r^{3}}(\mathbf{1}-3 \boldsymbol{e} \otimes \boldsymbol{e}) . & R(t)<r<\infty,\end{cases}
$$

A calculation shows that, for $\boldsymbol{S}$ as given by (13.7), $\operatorname{div} \boldsymbol{S}=\mathbf{0}$ and, bearing in mind (13.2b), the momentum balance (2.7) yields expressions for $\partial p / \partial r$ in both phases and, on integrating these expressions, we find that

$$
p(r, t)= \begin{cases}p^{-}(t), & 0<r<R(t), \\ p_{\infty}(t)+\frac{\varrho^{+}}{r}\left(\frac{\mathrm{d} Q(t)}{\mathrm{d} t}-\frac{Q^{2}(t)}{2 r^{3}}\right), & R(t)<r<\infty,\end{cases}
$$


where $p^{-}$denotes the uniform pressure in the $(-)$phase and $p_{\infty}$ denotes the assigned pressure in the far field.

Turning to the bulk consequences of energy balance, we note from (13.1), (13.2a,c), and (13.5) that

$$
\dot{\varepsilon}(\boldsymbol{x}, t)= \begin{cases}c^{-} \frac{\partial \vartheta(r, t)}{\partial t}, & 0<r<R(t), \\ c^{+}\left(\frac{\partial \vartheta(r, t)}{\partial t}+\frac{Q}{r^{2}} \frac{\partial \vartheta(r, t)}{\partial r}\right), & R(t)<r<\infty .\end{cases}
$$

Further, by (13.5) and (13.7),

$$
\mathbf{S}(\boldsymbol{x}, t): \boldsymbol{D}(\boldsymbol{x}, t)= \begin{cases}0, & 0<r<R(t), \\ \frac{12 \mu^{+} Q^{2}(t)}{r^{6}}, & R(t)<r<\infty\end{cases}
$$

and, since the conductivities of the phases are assumed constant,

$$
\boldsymbol{q}(\boldsymbol{x}, t)= \begin{cases}-k^{-} \frac{\partial \vartheta(r, t)}{\partial r} \boldsymbol{e}, & 0<r<R(t) \\ -k^{+} \frac{\partial \vartheta(r, t)}{\partial r} \boldsymbol{e}, & R(t)<r<\infty .\end{cases}
$$

In view of (13.9)-(13.11), the energy balance $(2.10 a)$ yields

$$
\left.\begin{array}{ll}
c^{-} \frac{\partial \vartheta}{\partial t}=k^{-}\left(\frac{\partial^{2} \vartheta}{\partial r^{2}}+\frac{2}{r} \frac{\partial \vartheta}{\partial r}\right), & 0<r<R, \\
c^{+}\left(\frac{\partial \vartheta}{\partial t}+\frac{Q}{r^{2}} \frac{\partial \vartheta}{\partial r}\right)=\frac{12 \mu^{+} Q^{2}}{r^{6}}+k^{+}\left(\frac{\partial^{2} \vartheta}{\partial r^{2}}+\frac{2}{r} \frac{\partial \vartheta}{\partial r}\right), & R<r<\infty .
\end{array}\right\}
$$

\subsection{Interface equations}

In addition to the temperature field $\vartheta$, our consideration of the bulk equations leaves undetermined the functions $Q, p^{-}$and $R$ of $t$. A full system of equations for the determination of $\vartheta, Q, p^{-}$and $R$ arises on considering the interface conditions (4.12), (4.15), (12.13) and either (12.14) or (12.15). We emphasize that (4.15) is automatically satisfied when $\boldsymbol{u}$ is as determined by (13.2a) and (13.5).

For a spherical interface with the phases arranged as assumed above,

$$
\boldsymbol{n}=\boldsymbol{e}, \quad V=\frac{\mathrm{d} R}{\mathrm{~d} t}, \quad K=-\frac{2}{r} .
$$

In view of (13.5), the mass balance (4.12) specializes to

$$
J=\varrho^{+}\left(\frac{\mathrm{d} R}{\mathrm{~d} t}-\frac{Q}{R^{2}}\right)=\varrho^{-} \frac{\mathrm{d} R}{\mathrm{~d} t},
$$

which yields

$$
Q=\frac{\llbracket \varrho \rrbracket R^{2}}{\varrho^{+}} \frac{\mathrm{d} R}{\mathrm{~d} t}=-\frac{\llbracket v \rrbracket R^{2}}{v^{-}} \frac{\mathrm{d} R}{\mathrm{~d} t} .
$$

Bearing in mind (13.11) and (13.14), the energy balance (12.13a) becomes

$$
-\llbracket k \frac{\partial \vartheta}{\partial r} \rrbracket=\frac{\ell}{v^{-}} \frac{\mathrm{d} R}{\mathrm{~d} t} .
$$


Next, turning to the momentum balance, we note from (13.5), (13.7) and (13.13)(13.15), that

$$
-\psi^{x} K \boldsymbol{n}=\frac{2 \psi^{x}}{R} \boldsymbol{e}, \quad \llbracket \boldsymbol{S} \rrbracket \boldsymbol{n}=\frac{4 \mu^{+} \llbracket v \rrbracket}{v^{-} R} \frac{\mathrm{d} R}{\mathrm{~d} t} \boldsymbol{e}, \quad J^{2} \llbracket v \rrbracket \boldsymbol{n}=\frac{\llbracket v \rrbracket}{\left(v^{-}\right)^{2}}\left(\frac{\mathrm{d} R}{\mathrm{~d} t}\right)^{2} \boldsymbol{e},
$$

Further, by (13.3) and (13.15),

$$
\mathbb{L}=\mathbb{D}=\mathbb{P}\left\langle\langle\boldsymbol{L}\rangle \mathbb{P}=\frac{1}{2} \mathbb{P} L^{+} \mathbb{P}=\frac{Q}{2 R^{3}} \mathbb{P}=-\frac{\llbracket v \rrbracket}{2 v^{-} R} \frac{\mathrm{d} R}{\mathrm{~d} t} \mathbb{P},\right.
$$

so that $2 \alpha \mathbb{D}+\lambda(\operatorname{tr} \mathbb{D}) \mathbb{P}=-(\alpha+\lambda)\left(\llbracket v \rrbracket / v^{-} R\right)(\mathrm{d} R / \mathrm{d} t) \mathbb{P}$ and thus

$$
\operatorname{div}_{\mathscr{S}}(2 \alpha \mathbb{D}+\lambda(\operatorname{tr} \mathbb{D}) \mathbb{P})=\frac{2(\alpha+\lambda) \llbracket v \rrbracket}{v^{-} R^{2}} \frac{\mathrm{d} R}{\mathrm{~d} t} \boldsymbol{e} .
$$

In view of (13.17) and (13.19), the momentum balance (2.13b) specializes to

$$
\llbracket p \rrbracket=\frac{2 \llbracket v \rrbracket}{v^{-} R}\left(2 \mu^{+}+\frac{\alpha+\lambda}{R}\right) \frac{\mathrm{d} R}{\mathrm{~d} t}-\frac{\llbracket v \rrbracket}{\left(v^{-}\right)^{2}}\left(\frac{\mathrm{d} R}{\mathrm{~d} t}\right)^{2}-\frac{2 \psi^{x}}{R} .
$$

Next, since $\left.V^{m i g}=\mathrm{d} R / \mathrm{d} t-Q / 2 R^{2}=(\langle v\rangle\rangle / v^{-}\right) \mathrm{d} R / \mathrm{d} t$ and $\Delta_{\mathscr{S}} V^{m i g}=0$, the normal combined balance (12.15) becomes

$$
\ell \frac{\vartheta-\vartheta_{0}}{\vartheta_{0}}=\llbracket v \rrbracket\left(\langle p\rangle-\frac{2 \mu^{+} \llbracket v \rrbracket}{v^{-} R} \frac{\mathrm{d} R}{\mathrm{~d} t}\right)-\frac{2\left\langle\langle v\rangle \psi^{x}\right.}{R}-\frac{\kappa\langle\langle v\rangle\rangle^{2}}{v^{-}} \frac{\mathrm{d} R}{\mathrm{~d} t} .
$$

Summarizing, the basic interface equations for radially symmetric flow - the energy balance, the momentum balance, and the normal combined balance - take the form

$$
\begin{gathered}
-\llbracket k \frac{\partial \vartheta}{\partial r} \rrbracket=\frac{\ell}{v^{-}} \frac{\mathrm{d} R}{\mathrm{~d} t}, \\
\llbracket p \rrbracket=\frac{4 \mu^{+} \llbracket v \rrbracket}{v^{-} R} \frac{\mathrm{d} R}{\mathrm{~d} t}-\frac{\llbracket v \rrbracket}{\left(v^{-}\right)^{2}}\left(\frac{\mathrm{d} R}{\mathrm{~d} t}\right)^{2}+\frac{2(\alpha+\lambda) \llbracket v \rrbracket}{v^{-} R^{2}} \frac{\mathrm{d} R}{\mathrm{~d} t}-\frac{2 \psi^{x}}{R}, \\
\ell \frac{\vartheta-\vartheta_{0}}{\vartheta_{0}}=\llbracket v \rrbracket\left(\langle p\rangle-\frac{2 \mu^{+} \llbracket v \rrbracket}{v^{-} R} \frac{\mathrm{d} R}{\mathrm{~d} t}\right)-\frac{2\left\langle\langle v\rangle \psi^{x}\right.}{R}-\frac{\kappa\left\langle\langle\rangle^{2}\right.}{v^{-}} \frac{\mathrm{d} R}{\mathrm{~d} t} .
\end{gathered}
$$

\subsection{Generalized Gibbs-Thomson relation}

Using (13.22b) to compute $p^{-}$and inserting the result along with (13.8) in (13.22c), we arrive at the generalized Gibbs-Thomson relation

$$
\begin{aligned}
& \ell \frac{\vartheta-\vartheta_{0}}{\vartheta_{0}}=-\frac{\llbracket v \rrbracket^{2}}{v^{+} v^{-}}\left(R \frac{\mathrm{d}^{2} R}{\mathrm{~d} t^{2}}+\frac{3}{2}\left(\frac{\mathrm{d} R}{\mathrm{~d} t}\right)^{2}\right) \\
& \quad-\frac{1}{v^{-}}\left(\frac{\llbracket v \rrbracket^{2}}{R}\left(4 \mu^{+}+\frac{\lambda+\alpha}{R}\right)+\kappa\langle v\rangle^{2}\right) \frac{\mathrm{d} R}{\mathrm{~d} t}-\frac{2 v^{-} \psi^{x}}{R}+\llbracket v \rrbracket p_{\infty} .
\end{aligned}
$$

\subsection{Free-boundary problem in terms of dimensionless quantities}

Assuming that the pressure at infinity $p_{\infty}$ is assigned, the radially summetric problem reduces to a free-boundary problem for the temperature field $\vartheta$ (in each of the bulk phases) and the radius $R$ of the interface. In particular, this problem consists of bulk equations which arise on using (13.15) in (13.12) along with the interface condition (13.22a), the generalized Gibbs-Thomson relation $(13.23)$, and the condition $\llbracket \vartheta \rrbracket=0$. 
Let $\vartheta_{\infty}$ denote the temperature in the far field, define a characteristic length scale

$$
r_{*}=\frac{2 v^{-} \psi^{x} \vartheta_{0}}{\ell\left(\vartheta_{0}-\vartheta_{\infty}\right)}
$$

and introduce dimensionless variables

$$
\bar{\theta}=\frac{\vartheta-\vartheta_{\infty}}{\vartheta_{0}-\vartheta_{\infty}}, \quad \bar{r}=\frac{r}{r_{*}}, \quad \bar{R}=\frac{R}{r_{*}}, \quad \bar{t}=\frac{k^{+} t}{c^{+} r_{*}^{2}} .
$$

Dropping bars, we then arrive at a dimensionless free-boundary problem consisting of the bulk equations

$$
\begin{aligned}
& \frac{\partial \theta}{\partial t}=a\left(\frac{\partial^{2} \theta}{\partial r^{2}}+\frac{2}{r} \frac{\partial \theta}{\partial r}\right) \quad(0<r<R), \\
& \frac{\partial \theta}{\partial t}-\frac{\Delta v R^{2}}{r^{2}} \frac{\mathrm{d} R}{\mathrm{~d} t} \frac{\partial \theta}{\partial r}=\frac{(\Delta v)^{2} S \mathscr{N}_{D} R^{4}}{r^{6}}\left(\frac{\mathrm{d} R}{\mathrm{~d} t}\right)^{2}+\frac{\partial^{2} \theta}{\partial r^{2}}+\frac{2}{r} \frac{\partial \theta}{\partial r} \quad(R<r<\infty),
\end{aligned}
$$

together with the interface equations

$$
\begin{aligned}
\theta= & \theta^{+}=\theta^{-} \\
\frac{1}{S} \frac{\mathrm{d} R}{\mathrm{~d} t}= & -\left(\frac{\partial \theta}{\partial r}\right)^{+}+k\left(\frac{\partial \theta}{\partial r}\right)^{-}, \\
\theta= & 1+\frac{\Delta v P}{S}-\frac{1}{R}-\mathscr{N}_{\kappa} \frac{\mathrm{d} R}{\mathrm{~d} t} \\
& -(\Delta v)^{2} S \mathscr{N}_{v}\left(\frac{1}{R} \frac{\mathrm{d} R}{\mathrm{~d} t}+\frac{1}{4 P r^{+}}\left(R \frac{\mathrm{d}^{2} R}{\mathrm{~d} t^{2}}+\frac{3}{2}\left(\frac{\mathrm{d} R}{\mathrm{~d} t}\right)^{2}\right)+\frac{S \mathscr{N}_{v i}}{R^{2}} \frac{\mathrm{d} R}{\mathrm{~d} t}\right),
\end{aligned}
$$

with dimensionless parameters defined via

$$
\begin{gathered}
\Delta v=\frac{\llbracket v \rrbracket}{v^{-}}, \quad a=\frac{c^{+} k^{-}}{c^{-} k^{+}}, \quad k=\frac{k^{-}}{k^{+}}, \quad P^{+}=\frac{v^{+} c^{+} \mu^{+}}{k^{+}}, \\
S=\frac{c^{+}\left(\vartheta_{0}-\vartheta_{\infty}\right) v^{-}}{\ell}, \quad P=\frac{\vartheta_{0} c^{+}\left(v^{-}\right)^{2} p_{\infty}}{\ell^{2}}, \quad \mathscr{N}_{\kappa}=\frac{\langle v\rangle^{2}}{\left(v^{-}\right)^{2}} \frac{\kappa k^{+}}{2 c^{+} \psi^{x}}, \\
\mathscr{N}_{\nu}=\frac{\mu^{+} k^{+} \ell^{2}}{\left(v^{+} \psi^{x}\right)^{2} c^{+} \vartheta_{0}}, \mathscr{N}_{v i}=\frac{(\lambda+\alpha) l^{2}}{8 \mu^{+}\left(v^{-}\right)^{2} \psi^{x} c^{+} \vartheta_{0}}, \quad \mathscr{N}_{D}=\frac{3 \mu^{+} k^{+} \ell^{3}}{\left(c^{+}\right)^{3} \vartheta_{0}^{2}\left(v^{-}\right)^{3}\left(\psi^{x}\right)^{2}} .
\end{gathered}
$$

Here, $\Delta v$ represents the density difference between phases, $a$ and $k$ are thermal property ratios, $\mathrm{Pr}^{+}$is a Prandtl number, $S$ is a Stefan number, $P$ is a dimensionless applied pressure, $\mathscr{N}_{\kappa}$ is a dimensionless kinetic coefficient, $\mathscr{N}_{\nu}$ and $\mathscr{N}_{v i}$ are dimensionless parameters associated with bulk viscosity and interface viscosity effects on the interface temperature, and $\mathscr{N}_{D}$ is a parameter associated with bulk viscous heating.

\subsection{Solution approach}

The problem (13.26)-(13.27) includes a variety of special cases for which similaritytype solutions have been obtained. Cases without flow include the classical Stefan problem with interfacial temperature equal to the melting temperature (Frank 1950), the Stefan problem with interfacial temperature modified by interfacial curvature (Krukowski \& Turski 1982) and the Stefan problem with interfacial curvature and 
attachment kinetic effects (Sekerka et al. 1988). The effect of advective fluid flow, driven by density change upon phase transformation, in the melt on the classical Stefan problem has been examined by Howison (1988). Curvature, kinetics, and hydrodynamic effects - such as bulk viscous dissipation or interfacial viscosity have yet to be treated. Our goal here is to highlight the new hydrodynamic effects contained in (13.26)-(13.27). To achieve this, we adopt an approach based on a generalized similarity-type solution.

We assume that

$$
R(t)=2 x_{0} \sqrt{t}
$$

with $x_{0}$ a constant to be determined, and introduce a change of variables of the form

$$
\theta(r, t)=\sum_{\alpha=0}^{3} \frac{\Theta_{\alpha}(x)}{r^{\alpha}}, \quad x=\frac{r}{2 \sqrt{t}} .
$$

The various powers of $r$ appearing in the expansion (13.30a) are required to satisfy the bulk and interfacial equations. Using the change of variables $(13.30 a, b)$ in the bulk equations (13.30), we find that, for each $\alpha=0,1,2,3, \Theta_{\alpha}$ must obey

$$
\frac{\mathrm{d}^{2} \Theta_{\alpha}}{\mathrm{d} x^{2}}+2\left(\frac{1-\alpha}{x}+\frac{x}{a}\right) \frac{\mathrm{d} \Theta_{\alpha}}{\mathrm{d} x}+\frac{\alpha(\alpha-1)}{x^{2}} \Theta_{\alpha}=0,
$$

for $0<x<x_{0}$, and

$$
\begin{aligned}
& \frac{\mathrm{d}^{2} \Theta_{\alpha}}{\mathrm{d} x^{2}}+2\left(\frac{1-\alpha}{x}+x+\frac{x_{0}^{3} \Delta v}{x^{2}}\right) \frac{\mathrm{d} \Theta_{\alpha}}{\mathrm{d} x}+\frac{\alpha}{x^{2}}\left(\alpha-1-\frac{2 x_{0}^{3} \Delta v}{x}\right) \Theta_{\alpha} \\
& =-\frac{4 S \mathscr{N}_{D}(\Delta v)^{2} x_{0}^{6} \delta_{\alpha 2}}{x^{4}},
\end{aligned}
$$

for $x>x_{0}$. Similarly, using (13.30) in (13.27), we find that, for each $\alpha=0,1,2,3$,

$$
\begin{aligned}
\Theta_{\alpha}^{+}= & \Theta_{\alpha}^{-}, \\
\frac{2 x_{0} \delta_{\alpha 0}}{S}= & -\left(\frac{\mathrm{d} \Theta_{\alpha}}{\mathrm{d} x}-\frac{\alpha \Theta_{\alpha}}{x}\right)^{+}+k\left(\frac{\mathrm{d} \Theta_{\alpha}}{\mathrm{d} x}-\frac{\alpha \Theta_{\alpha}}{x}\right)^{-} \\
\Theta_{\alpha}= & \left(1+\frac{P \Delta v}{S}\right) \delta_{\alpha 0}-\left(1+2 x_{0}^{2} \mathscr{N}_{\kappa}\right) \delta_{\alpha 1} \\
& -2 x_{0}^{2}(\Delta v)^{2} S \mathscr{N}_{\nu}\left(1+\frac{x_{0}^{2}}{4 P r^{+}}\right) \delta_{\alpha 2}-2 x_{0}^{2}(\Delta v)^{2} S^{2} \mathscr{N}_{v} \mathscr{N}_{v i} \delta_{\alpha 3},
\end{aligned}
$$

at $x=x_{0}$. In (13.32)-(13.33), $\delta_{\alpha \beta}$ denotes the Kronecker delta.

The quantity $\Theta_{0}$ can be determined explicitly and is given by $\Theta_{0}=(1+P \Delta v / S)$ for $x<x_{0}$ and $\Theta_{0}(x)=(1+P \Delta v / S) F_{0}(x) / F_{0}\left(x_{0}\right)$ for $x>x_{0}$, with

$$
F_{0}(x)=\int_{x}^{\infty} \exp \left(-y^{2}+\frac{2 x_{0}^{3} \Delta v}{y}\right) \frac{\mathrm{d} y}{y^{2}} .
$$

The quantity $x_{0}$ is required to satisfy

$$
2 x_{0}^{3} F_{0}\left(x_{0}\right) \exp \left((1-2 \Delta v) x_{0}^{2}\right)=S+P \Delta v .
$$

The remaining quantities $\Theta_{1}, \Theta_{2}$ and $\Theta_{3}$ were obtained by numerical integration. In particular, in the (-) phase the ordinary differential equations (13.31) were integrated numerically starting from the centre of the liquid sphere - with the aid of a series expansion for $x \ll 1$ to start the integration away from the origin - up to the interface 
Dimensional parameters

$\begin{array}{lccc}\text { Density of water } \dagger & \varrho^{-}=1 / v^{-} & 481.6 & \mathrm{~kg} \mathrm{~m}^{-3} \\ \text { Density of steam } \dagger & \varrho^{+}=1 / v^{+} & 177.4 & \mathrm{~kg} \mathrm{~m}^{-3} \\ \text { Dynamic viscosity of water } \dagger & \mu^{-} & 5.526 \times 10^{-5} & \mathrm{~Pa} \mathrm{~s} \\ \text { Dynamic viscosity of steam } \dagger & \mu^{+} & 2.795 \times 10^{-5} & \mathrm{~Pa} \mathrm{~s} \\ \text { Thermal conductivity of water } \dagger & k^{-} & 0.4177 & \mathrm{~W} \mathrm{mK}^{-1} \\ \text { Thermal conductivity of steam } \dagger & k^{+} & 0.2499 & \mathrm{~W} \mathrm{mK}^{-1} \\ \text { Thermal diffusivity of water } \dagger & k^{-} / c^{-} & 3.276 \times 10^{-8} & \mathrm{~m}^{2} \mathrm{~s}^{-1} \\ \text { Thermal diffusivity of steam } \dagger & k^{+} / c^{+} & 2.682 \times 10^{-8} & \mathrm{~m}^{2} \mathrm{~s}^{-1} \\ \text { Surface energy } & \psi^{x} & 8.09 \times 10^{-4} & \mathrm{~J} \mathrm{~m}^{-2} \\ \text { Kinetic coefficient }+ & \kappa & 10^{5} & \mathrm{~N} \mathrm{~s} \mathrm{~m}^{-3} \\ \text { Latent heatt } & l & 5.524 \times 10^{5} & \mathrm{~J} \mathrm{~kg}^{-1} \\ \text { Interfacial viscosity } & \lambda+\alpha & 10^{-11}-10^{-7} & \mathrm{~N} \mathrm{~s} \mathrm{~m}^{-1} \\ \text { Equilibrium temperature (at } p=202.7 \text { bar) } & \vartheta_{0} & 640 & \mathrm{~K}^{-}\end{array}$

TABLE 1. Thermophysical properties of the steam-water system at the reference pressure 202.7 bar and the corresponding equilibrium temperature $640 \mathrm{~K}$ (Parry 2000 $†$ ). The critical temperature and pressure for the steam-water system are $647 \mathrm{~K}$ and $221.1 \mathrm{bar}$ and the critical density is $323 \mathrm{~kg} \mathrm{~m}^{-3}$. An estimate for a kinetic coefficient for solid-liquid systems is contained in the expression $V=V_{0} \Delta S_{f} \Delta T / \bar{N} k_{B} T_{i}$ (Coriell \& Turnbull 1982‡) where $V$ is the interfacial normal velocity, $V_{0}$ is the liquid sound speed, $\Delta S_{f}$ is the molar entropy of fusion at the interfacial temperature $T_{i}, \Delta T=T_{i}-T_{M}$ where $T_{M}$ is the thermodynamic equilibrium temperature, $\bar{N}$ is the Avogadro number and $k_{B}$ is the Boltzmann constant. Therefore, with $\Delta T / T_{i}$ taken as $\left(\vartheta-\vartheta_{0}\right) / \vartheta_{0}$ an estimate for $\kappa$ follows from $\kappa=l \bar{N} k_{B} / V_{0} \Delta S_{f}\langle\langle v\rangle$. Using $V_{0} \approx 1000 \mathrm{~m} \mathrm{~s}^{-1}, \Delta S_{f}=15.55 \mathrm{~J} \mathrm{~K}^{-1} \mathrm{~mol}^{-1}$, and $\langle u\rangle=3.85 \times 10^{-3} \mathrm{~m}^{-3} \mathrm{~kg}^{-1}$, we find that $\kappa=8 \times 10^{4} \mathrm{~N} \mathrm{~s} \mathrm{~m}^{-3}$. The estimate for interfacial viscosity is obtained from Scriven $\&$ Sternling (1963). Specifically, they define $N_{v i}=(\lambda+\alpha) / \mu d$, where $d$ is a fluid-layer thickness and $\mu$ is the fluid viscosity and note that for a fluid layer of thickness $1 \mathrm{~mm}, \mathscr{N}_{v i}$ ranges from $10^{-3}$ (clean surface) to 10 (surface with insoluble monolayer). Therefore, using $\mu \approx 10^{-5} \mathrm{Pas}$ we find that $\lambda+\alpha=\mathscr{N}_{v i} \mu d$ ranges approximately between $10^{-11}$ and $10^{-7}$.

$x=x_{0}$. The linearity of (13.31) allowed a rescaling of the computed value of $\Theta_{\alpha}$ to match the correct temperature conditions at $x=x_{0}$ as determined by the modified Gibbs-Thomson condition $(13.33 c)$. The remaining conditions $(13.33 a, b)$ provided starting conditions for the integration of the the ordinary differential equations (13.32) in the $(+)$ phase. These equations were integrated from $x=x_{0}$ to a fixed far-field value $x=x_{\max }$. A value of $x_{\max }=6$ was used for the calculations. The numerical approach was verified by comparison to analytical series solutions in the bulk phase and also by comparison to the results of Sekerka et al. (1988) for the analogous problem without fluid flow. The effects associated with the new features under investigation in the present work are illustrated and discussed below.

\subsection{Discussion}

We have performed numerical calculations using thermophysical properties based on the water-steam system below the critical point (Wagner 1998; Parry 2000). The material constants are evaluated at an equilibrium reference state of constant pressure and temperature; in this section, $\vartheta_{0}$ denotes the equilibrium temperature at the reference pressure (not at the unstressed state) and $p_{\infty}$ denotes the far-field applied pressure relative to the reference pressure. The assumed thermophysical properties are given in table 1, with the corresponding dimensionless parameters in table 2. 


$\begin{array}{cllllll} & \text { Case 1 } & \text { Case 2 } & \text { Case 3 } & \text { Case 4 } & \text { Case 5 } & \text { Case 6 } \\ \Delta v & 0 & 0 & 1.715 & 1.715 & 1.715 & 1.715 \\ a & 1.221 & 1.221 & 1.221 & 1.221 & 1.221 & 1.221 \\ k & 1.671 & 1.671 & 1.671 & 1.671 & 1.671 & 1.671 \\ \mathscr{N}{ }_{k} & 0 & 1 & 0 & 1 & 1 & 1 \\ P^{+} & \text {n/a } & \text { n/a } & \text { n/a } & \text { n/a } & 5.875 & 5.875 \\ \mathscr{N}_{v} & \text { n/a } & \text { n/a } & 0.0 & 0.0 & 13.6 & 13.6 \\ \mathscr{N}_{v i} & \text { n/a } & \text { n/a } & 0.0 & 0 & 0 & 10.0 \\ S & 0.082 & 0.082 & 0.076 & 0.076 & 0.076 & 0.076 \\ x_{0} & 0.25 & 0.25 & 0.25 & 0.25 & 0.25 & 0.25\end{array}$

TABLE 2. Dimensionless material parameters used in the numerical calculations. The non-zero values of the parameters $\Delta v, a, k, \mathrm{Pr}^{+}$and $\mathscr{N}_{v}$ follow from the dimensional parameters in table 1 . Owing to the broad uncertainty in the appropriate values for parameters $\mathscr{N}_{\kappa}$ and $\mathscr{N}_{\nu i}$, we have chosen values for these parameters with the goal of illustrating their qualitative effects. Note that the values used for $\mathrm{Pr}^{+}, \mathscr{N}_{v}$ and $\mathscr{N}_{v i}$ are irrelevant to the solution when $\Delta v=0$. Case 1: parameters showing the reference case with capillarity but no flow or kinetics. Case 2: parameters showing reference case with with capillarity and kinetics but no flow. Case 3: fluid flow with capillarity but no kinetics. Case 4: fluid flow with capillarity and kinetics. Case 5: fluid flow with capillarity, kinetics, and viscosity. Case 6: fluid flow with capillarity, kinetics, viscosity, and interfacial viscosity.

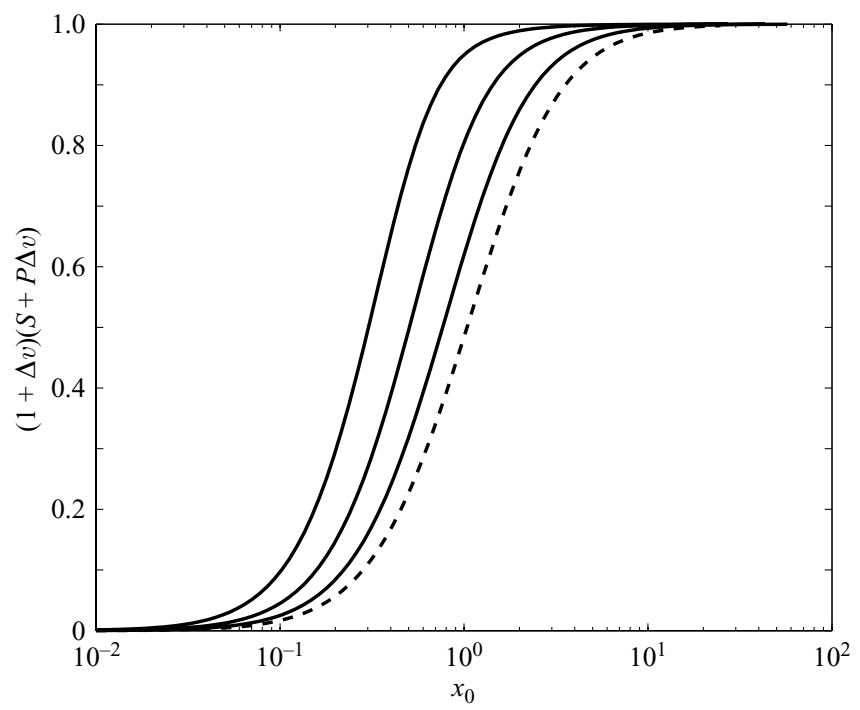

FIGURE 1. Undercooling versus the similarity variable $x_{0}$ for various density ratios $\Delta v$. Here the interface position is given by $r=2 x_{0} \sqrt{t}$. From right to left, the curves correspond to $\Delta v=0$ (dashed curve), $\Delta v=0.5, \Delta v=1.715$, and $\Delta v=5.0$. All variables are dimensionless.

Figure 1 illustrates the dependence of $x_{0}$ on the undercooling for different values of the dimensionless difference $\Delta v$ of the specific volume. Note that

$$
(1+\Delta v)(S+P \Delta v)=\frac{c^{+} v^{+} \vartheta_{0}}{\ell}\left(1+\frac{p_{\infty} \llbracket v \rrbracket}{\ell}-\frac{\vartheta_{\infty}}{\vartheta_{0}}\right)
$$

represents the dimensionless undercooling based on the properties of the exterior phase. Here $\vartheta_{0}\left(1+p_{\infty} \llbracket v \rrbracket / \ell\right)$ is the equilibrium temperature of the interface, which 


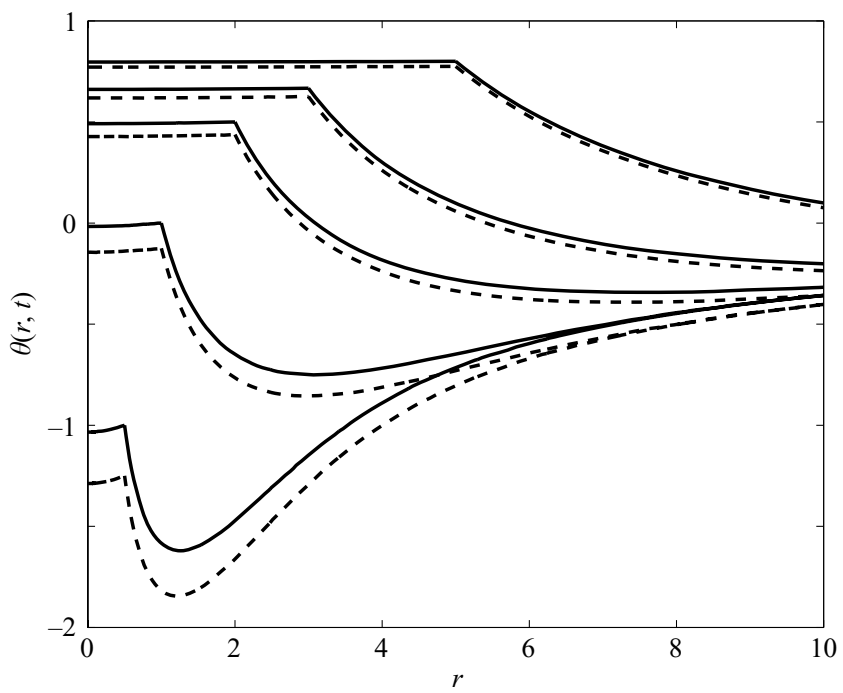

FIgURE 2. The temperature profiles $\theta(r, t)$ versus the radius $r$ at various times for Cases 1 (solid curves) and 2 (dashed curves) of table 2. From bottom to top, the various stages for each case have $R=0.5,1,2,3$ and 5 , which correspond to $t=1,4,16,36$ and 100, so that $R(t)=0.5 \sqrt{t}$. All variables are dimensionless.

includes the shift in temperature with pressure due to the Clausius-Clapeyron effect (Callen 1960). Positive $\Delta v$ corresponds to a less dense (+) phase, which is the usual situation for a liquid surrounded by its vapour. For a given undercooling, there is a significant reduction in interfacial motion for large positive values of $\Delta v$. This trend is similar to that obtained for the effect of a density-change flow on the growth of a dendrite tip (McFadden \& Coriell 1986). For dimensionless undercoolings greater than unity there are presumably no spherically symmetric solutions that grow diffusively (that is, for which $R(t) \sim \sqrt{t}$ ) (Frank 1950).

As discussed by Sekerka et al. (1988), the solution describing diffusive growth with capillarity and kinetics is driven by a significant initial undercooling in the sample, which distinguishes this case from the classical theory of nucleation and growth in an isothermal system. This initial undercooling is evident in figure 2, where thermal profiles for growth with capillarity effects without kinetics (solid curves, Case 1 in table 2), and both capillarity and kinetic effects (dashed curves, Case 2 in table 2) are shown for $x_{0}=0.25$; cf. figures 2 and 3 in Sekerka et al. (1988). For small times, the effect of capillarity is to lower the temperature of the spherical interface relative to that of a planar interface $(\theta=1)$; for long times, the radius becomes large enough so that the effects of capillarity become insignificant. For $\mathscr{N}_{\kappa}=0$, interfacial attachment kinetics are infinitely rapid; with finite attachment kinetics $\left(\mathscr{N}_{\kappa}>0\right)$, interfacial motion is more sluggish and a finite deviation in the interfacial temperature from its equilibrium value is necessary to drive the interfacial motion. Thus, the corresponding temperatures for the case $\mathscr{N}_{\kappa}=1$ are lower than those for $\mathscr{N}_{\kappa}=0$.

In figure 3, we illustrate the effects of an applied pressure on the system by plotting temperature profiles at a fixed time. Here, with a finite change in density $(\Delta v>0)$, the equilibrium interfacial temperature is raised by an applied overpressure relative to the reference pressure and - due to the Clausius-Clapeyron effect - lowered by an applied 


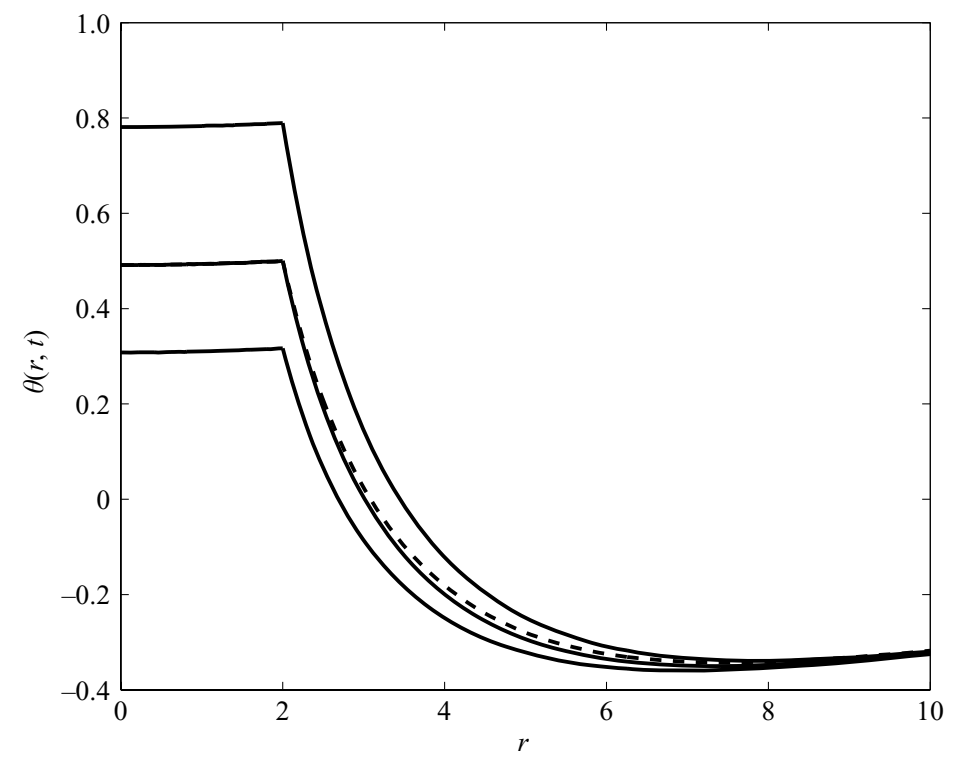

FIGURE 3. The temperature profiles $\theta(r, t)$ versus the radius $r$ for various applied pressures $P$ at time $t=16$. From top to bottom, the solid curves correspond to Case 3 but with the pressure set to $P=0.01(S=0.059), P=0(S=0.076)$, and $P=-0.01(S=0.094)$, respectively. For comparison, Case 1 with $P=0$ is also included as the dashed curve, which lies very close to the middle solid curve for $P=0$. All variables are dimensionless.

under pressure. For the material properties corresponding to steam given in table 1, the effect of the bulk density change flow on the temperature profiles is small, as illustrated by comparison with the dashed curve in figure 3 corresponding to $\Delta v=0$ (Case 1). For this type of flow, the effects of bulk viscous heating are also very small in this case, with $S \mathscr{N}_{D}(\Delta v)^{2} x_{0}^{6}=10^{-4}$. The heating tends to raise the temperature in the system slightly by amounts of this magnitude, and since the results are smaller than graphical precision, we have neglected the effects of bulk viscous heating in our numerical calculations. This effect is usually insignificant under normal conditions and is often neglected through the Boussinesq approximation (Chandrasekhar 1961) in studies of thermal transport.

The effects of flow, bulk viscosity and interfacial viscosity on the temperature profiles near the interface at a given time are shown in figure 4 . These curves all correspond to cases with finite attachment kinetics $\left(\mathscr{N}_{\kappa}=1\right)$ and interfacial capillarity. For $\Delta v>0$, the flow driven by the density change is toward the interface and tends to steepen the temperature gradient in the exterior phase at the interface, as can be seen by comparing the top two curves in figure 4 . With both attachment kinetics and the effect of bulk viscosity on the interfacial temperature taken into account, the temperatures are lowered further. Adding the effects of interfacial viscosity causes a comparable shift in the temperature profiles for this system. The lowering of the interfacial temperatures is consistent with a dissipation argument: when additional dissipation is present at the interface, less free energy is available to drive the interfacial motion, and so the driving temperature difference $\vartheta-\vartheta_{0}$ must increase to compensate for the dissipative energy loss. 


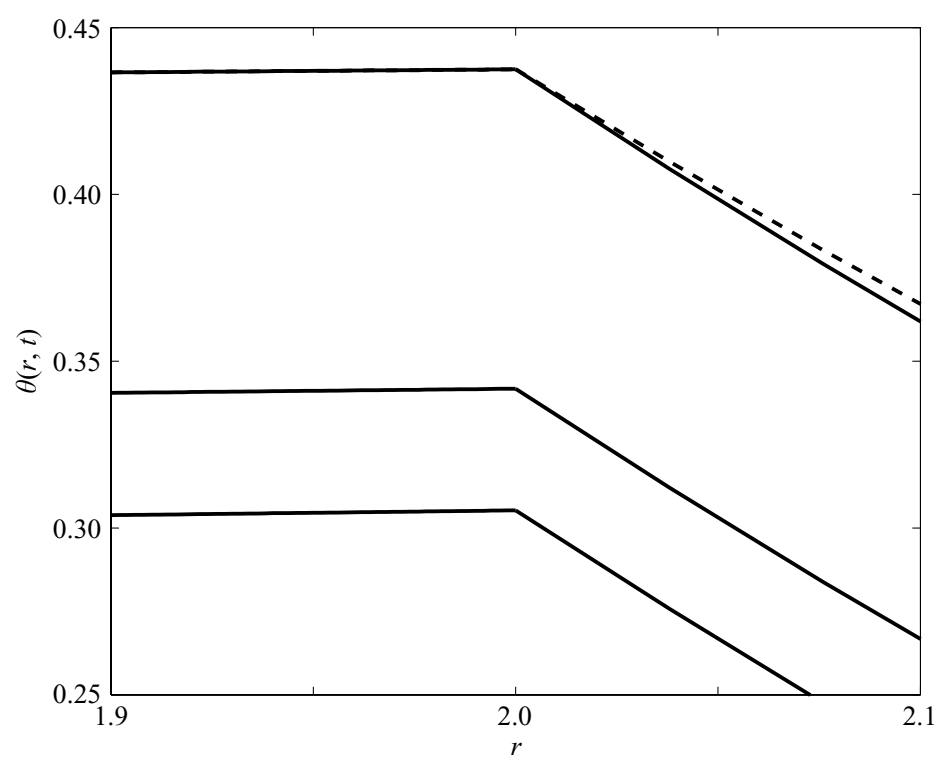

FIGURE 4. The temperature profiles $\theta(r, t)$ versus the radius $r$ near the interface at time $t=16$ for various cases in table 2. From top to bottom, the curves correspond to Cases 2 (dashed curve), 4, 5 and 6 . All variables are dimensionless.

\section{Summary}

We have developed a complete set of boundary conditions that apply at an interface between two fluid phases undergoing transformation. Our focus has been on those interfacial conditions whose equilibrium forms are well known, but whose extensions to settings with even simple fluid flows have not been firmly established. We have employed an approach based on a consideration of configurational forces in the context of non-equilibrium thermodynamics. This approach, which is well developed in the framework of solid mechanics, is extended here for fluids with both bulk viscosity and interfacial viscosity. Our results illuminate a number of physical effects that may play key roles for transport during phase transformation and interfacial flows.

We have illustrated our results by applying the theory to the condensation of a liquid drop growing into its vapour phase. For this problem, our model admits a radially symmetric solution with the liquid-vapour interface position growing with the square root of time. Since the densities of the liquid and vapour phases are unequal, conservation of mass at the moving interface generates a radial flow in the vapour phase and the thermal transport is altered by that flow. Whereas the velocity field can be obtained analytically, the temperature fields in both phases can be written as the sum of terms that each have a similarity form. The resulting ordinary differential equations are solved numerically, and the numerically generated solutions are combined to provide a temperature field satisfying the interfacial conditions arising from our model. The solution thus incorporates effects associated with the flow driven by a density change, interfacial energy, interfacial attachment kinetics, and both the bulk and the interfacial viscosities. The effects of applied pressure variations on the interfacial temperature through the Clausius-Clapeyron equation are also included in the model. The results show that the effects of the fluid flow on thermal transport 
in the bulk vapour phase are modest, but that the effects of capillarity, attachment kinetics, and bulk and interfacial viscosities are all significant near the liquid-vapour interface. These effects are particularly pronounced at early times when the interface radius is relatively small.

This work was supported in part by the Italian MIUR project 'Modelli Matematici per la Scienza dei Materiali' (P.C.), the US Department of Energy (E. F. and M. G.), the US National Science Foundation (D.M.A.), and the US NASA Microgravity Research Division (G. B. M.). The authors thank Xuemei Chen for her careful reading of various drafts of this paper. We also thank the referees for many helpful comments and suggestions.

\section{REFERENCES}

Anderson, D. M., McFadden, G. B. \& Wheeler, A. A. 1998 Diffuse-interface methods in fluid mechanics. Annu. Rev. Fluid Mech. 30, 139-165.

Anderson, D. M., McFadden, G. B. \& Wheeler, A. A. 2000 A phase-field model of solidification with convection. Physica D 135, 175-194.

Anderson, D. M., McFadden G. B. \& Wheeler, A. A. 2001 A phase-field model with convection: sharp-interface asymptotics. Physica D 151, 305-331.

Angenent, S. \& Gurtin, M. E. 1989 Multiphase thermomechanics with interfacial structure 2. Evolution of an isothermal interface. Arch. Rat. Mech. Anal. 108, 323-391.

Burelbach, J. P., Bankoff, S. G. \& Davis, S. H. 1988 Nonlinear stability of evaporating/condensing liquid films. J. Fluid Mech. 195, 463-494.

Caginalp, G. 1986 An analysis of a phase field model of a free boundary. Arch. Rat. Mech. Anal. 92, 205-245.

Caginalp, G. 1989 Stefan and Hele-Shaw type models as asymptotic limits of the phase-field equations. Phys. Rev. A 39, 5887-5896.

Callen, H. B. 1960 Thermodynamics. Wiley.

Cermelli, P. \& Fried, E. 1997 The influence of inertia on the configurational forces in a deformable solid. Proc. R. Soc. Lond. A 453, 1915-1927.

Cermelli, P., Fried, E. \& Gurtin, M. E 2005 Transport relations for surface integrals arising in the formulation of balance laws for evolving fluid interfaces. J. Fluid Mech. 544, 339-351.

Chandrasekhar, S. 1961 Hydrodynamic and Hydromagnetic Stability. Oxford University Press.

Collins, J. B. \& Levine, H. 1985 Diffuse interface model of diffusion-limited crystal growth. Phys. Rev. B 31, 6119-6122.

Coriell, S. R. \& Turnbull, D. 1982 Relative roles of heat transport and interface rearrangement rates in the rapid growth of crystals in undercooled melts. Acta Metall. 30, 2135-2139.

Danov, K. D., Alleborn, N., Raszillier, H. \& Durst, F. 1998 The stability of evaporating thin liquid films in the presence of surfactant. I. Lubrication approximation and linear analysis. Phys. Fluids 10, 131-143.

Edwards, D. A., Brenner, H. \& Wasan, D. T. 1991 Interfacial Transport Processes and Rheology. Butterworth-Heinemann.

Eshelby, J. D. 1951 The force on an elastic singularity. Phil. Trans. R. Soc. Lond. A 244, 87-112.

Eshelby, J. D. 1956 The continuum theory of lattice defects. In Progress in Solid State Physics 3 (ed. F. Seitz D. Turnbull). Academic.

EshelBy, J. D. 1970 Energy relations and the energy-momentum tensor in continuum mechanics. In Inelastic Behavior of Solids (ed. M. F. Kanninen, W. F. Alder, A. R. Rosenfield \& R. I. Jaffe). McGraw-Hill.

Eshelby, J. D. 1975 The elastic energy-momentum tensor. J. Elasticity 5, 321-335.

Frank, F. C. 1950 Radially symmetric phase growth controlled by diffusion. Proc. R. Soc. Lond. A 201, 586-599.

Frank, F. C. 1958 On the kinematic theory of crystal growth and dissolution processes. In Growth and Perfection of Crystals (ed. R. H. Doremus, B. W. Roberts \& D. Turnbull), Wiley. 
FRIED, E. 1995 Energy release, friction, and supplemental relations at phase interfaces. Continuum Mech. Thermodyn. 7, 111-121.

Fried, E. \& GuRTIN M. E. 1993 Continuum theory of thermally induced phase transitions based on an order parameter. Physica D 68, 326-342.

Fried, E. \& Gurtin M. E. 1999 Coherent solid-state phase transitions with atomic diffusion: a thermomechanical treatment. J. Statist. Phys. 95, 1361-1427.

GurTin, M. E. 1988 Multiphase thermomechanics with interfacial structure 1. Heat conduction and the capillary balance law. Arch. Rat. Mech. Anal. 104, 185-221.

Gurtin, M. E. 1995 The nature of configurational forces. Arch. Rat. Mech. Anal. 131, 67-100.

Gurtin, M. E. 2000 Configurational Forces as Basic Concepts of Continuum Physics. Springer.

Gurtin, M. E. \& Struthers, A. 1990 Multiphase thermomechanics with interfacial structure 3. Evolving phase boundaries in the presence of bulk deformation. Arch. Rat. Mech. Anal. 112, $97-160$.

Gurtin, M. E., Struthers, A. \& Williams, W. O. 1989 A transport theorem for moving interfaces. Q. Appl. Maths 47, 773-777.

Herring, C. 1951 Surface tension as a motivation for sintering. In The Physics of Powder Metallurgy (ed. W. E. Kingston). McGraw-Hill.

Hickman, K. C. D. 1952 Surface behavior in the pot still. Ind. Engng Chem. 44, 1892-1902.

Hickman, K. 1972 Torpid phenomena and pump oils. J. Vacuum Sci. Technol. 9, 960-976.

Howison, S. D. 1988 Similarity solutions to the Stefan problem and the binary alloy problem. IMA J. Appl. Maths 40, 147-161.

Joseph, D. D. \& Renardy, Y. 1993 Fundamentals of Two-Fluid Dynamics. Part 1: Mathematical Theory and Applications. Springer.

Krukowski, S. \& TURSKI, L. A. 1982 Time-dependent solution for spherically symmetric freezing precipitate. J. Cryst. Growth 58, 631-635.

Lamé, G. \& Clapeyron, B. P. 1831 Mémoire sur la solidification par refroidissement d'un globe liquide. Ann. Chim. Physique 47, 250-256.

LaPlace, P. S. 1806 Méchanique Céleste, Supplement au $\mathrm{X}^{\mathrm{e}}$ Livre, Impresse Imperiale, Paris. (Translated as Celestial Mechanics, vol. 4, Chelsea, New York, 1966.)

McFadden, G. B. \& CoRiell, S. R. 1986 The effect of fluid flow due to the crystal-melt density change on the growth of a parabolic dendrite. J. Cryst. Growth 74, 507-512.

MaXwell, J. C. 1875 On the dynamical evidence of the molecular constituion of matter. Nature 11, $357-359 \&$ 374-377.

Mullins, W. W. 1956 Two-dimensional motion of idealized grain boundaries. J. Appl. Phys. 27, 900-904.

NABARro, F. R. N. 1985 Material forces and configurational forces in interaction of elastic singularities. In Proc. Intl Symp. on the Mechanics of Dislocations (ed. E. C. Aifantis \& J. P. Hirth). American Society of Metals, Metals Park, OH.

Palmer, H. J. 1976 The hydrodynamic stability of rapidly evaporating liquids at reduced pressure. J. Fluid Mech. 75, 487-511.

PARry, W. T. 2000 ASME International Steam Tables for Industrial Use. ASME.

Peach, M. O. \& Koehler, J. S. 1950 The forces exerted on dislocations and the stress fields produced by them. Phys. Rev. 80, 436-439.

Penrose, O. \& Fife, P. C. 1990 Thermodynamically consistent models of phase-field type for the kinetics of phase-transitions. Physica D 43, 44-62.

Schrage, R. W. 1953 A Theoretical Study of Interphase Mass Transfer. Columbia University Press.

SCriven, L. E. 1960 Dynamics of a fluid interface. Chem. Engng Sci. 12, 98-108.

SCriven, L. E. \& Sternling, C. V. 1963 On cellular convection driven by surface-tension gradients: effects of mean surface tension and surface viscosity. J. Fluid Mech. 19, 321-340.

Sekerka, R. F., Voorhees, P. W., Coriell, S. R. \& McFadden, G. B. 1988 Initial conditions implied by $t^{1 / 2}$ solidification of a sphere with capillarity and interfacial kinetics. J. Cryst. Growth 87 , 415-420.

Slattery, J. C. 1990 Interfacial Transport Phenomena. Springer.

Stefan, J. 1889 Über einige Probleme der Theorie der Wärmeleitung. Sb. Kais. Akad. Wiss., Wien, Math.-Naturwiss. 98, 473-484. 
Stone, H. A., Stroock, A. D. \& AdJari, A. 2004 Engineering flows in small devices: microfluidics toward lab-on-a-chip. Annu. Rev. Fluid Mech. 36, 381-411.

Voronkov, V. V. 1964 Conditions for formation of mosaic structure on a crystallization front. Sov. Phys. Solid State 6, 2378-2381.

WAGNer, W. 1998 Properties of Water and Steam. Springer.

Wang, S. L., Sekerka, R. F., Wheeler, A. A., Murray, B. T., Coriell, S. R., Braun, R. J. \& McFadDEN, G. B. 1993 Thermodynamically-consistent phase-field models for solidification. Physica D 69, 189-200.

Young, T. 1805 An essay on the cohesion of fluids. Phil. Trans. R. Soc. Lond. 95, 65-87. 